\title{
Neural Network Potential Energy Surfaces for Small Molecules and Reactions
}

\author{
Sergei Manzhos ${ }^{1}$ and Tucker Carrington $\mathrm{Jr}^{2}$ \\ ${ }^{1}$ Centre Énergie Matériaux Télécommunications, Institut National de la Recherche Scientifique, \\ 1650, boulevard Lionel-Boulet, Varennes QC J3X1S2 Canada. E-mail: \\ sergei.manzhos@emt.inrs.ca \\ ${ }^{2}$ Chemistry Department, Queen’s University, Kingston ON K7L 3N6 Canada. E-mail: \\ tucker.carrington@queensu.ca
}

\begin{abstract}
We review progress in neural network $(\mathrm{NN})$ based methods for the construction of interatomic potentials from discrete samples (such as ab initio energies) for applications in classical and quantum dynamics including reaction dynamics and computational spectroscopy. The main focus is on methods for building molecular potential energy surfaces (PES) in internal coordinates that explicitly include all many-body contributions, even though some of the methods we review limit the degree of coupling, due either to a desire to limit computational cost or to limited data. Explicit and direct treatment of all many-body contributions is only practical for sufficiently small molecules, which are therefore our primary focus. This includes small molecules on surfaces. We consider direct, single NN PES fitting as well as more complex methods which impose structure (such as a multi-body representation) on the PES function, either through the architecture of one NN or by using multiple NNs. We show how NNs are effective in building representations with low-dimensional functions, including dimensionality reduction. We consider NN based approaches to build PESs in the sums-of-product form important for quantum dynamics, ways to treat symmetry, and issues related to sampling data distributions and the relation between PES errors and errors in observables. We highlight combinations of NNs with other ideas such as permutationally invariant polynomials or sums of environment-dependent atomic contributions which have recently emerged as powerful tools for building highly accurate PESs for relatively large molecular and reactive systems.
\end{abstract}




\section{Contents}

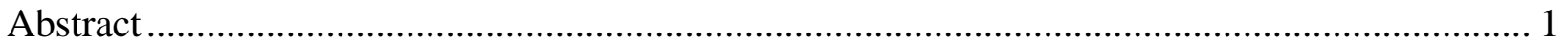

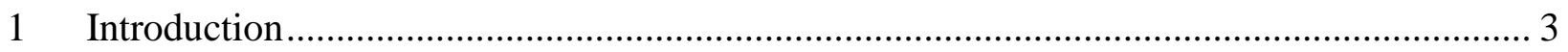

1.1 Molecular potential energy surfaces ................................................................ 3

1.2 Major PES fitting methods ............................................................................ 4

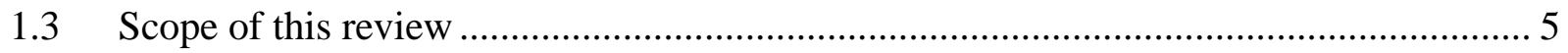

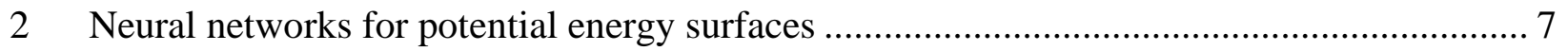

2.1 Introduction to neural networks used in PES fitting .............................................. 7

2.2 Achieving different types of representations via choice of the NN architecture or a

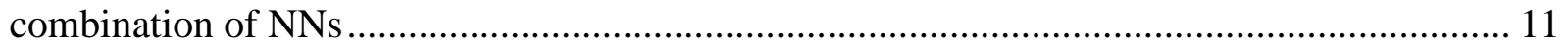

2.3 Symmetry, asymptotics and extrapolation ...................................................... 18

3 Applications of neural networks to build potential energy surfaces............................... 20

3.1 Brief timeline of neural network based potential energy surfaces: from a curiosity to

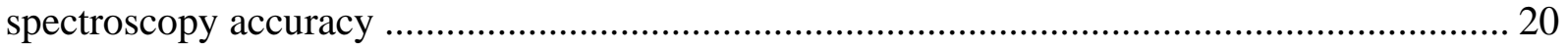

3.2 Neural network PESs for classical and quantum dynamics ..................................... 22

3.2.1 Neural network potentials for classical dynamics ......................................... 22

3.2.2 Neural network potentials for quantum dynamics and vibrational spectroscopy ... 27

S.3 SOP NN

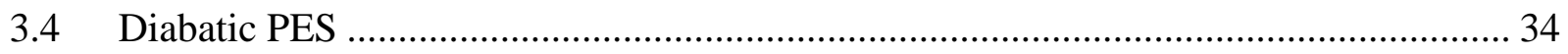

3.5 Representations in orders of coupling and with sub-dimensional component functions37

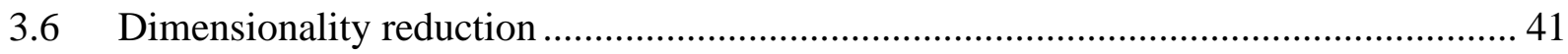

3.7 Data selection and meaningfulness of fitting and test errors .................................. 43

3.8 Controlled comparisons of NNs to other approaches............................................ 49

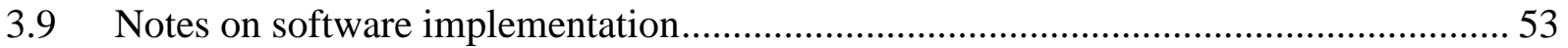

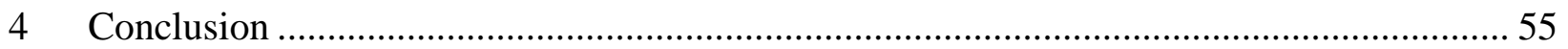

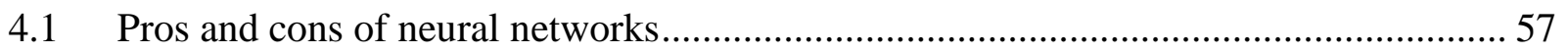


6 Abbreviations.

7 References

61

\section{Introduction}

\subsection{Molecular potential energy surfaces}

The nuclei of a molecule or a reacting system move in response to a potential. At each geometry (shape) of the nuclei, a potential value can be obtained by solving the electronic Schrödinger equation. The simplest conceptual recipe for computing observables related to the motion of the nuclei, e.g., rate constants, spectra, etc, requires solving the electronic Schrödinger equation at each nuclear geometry. This, however, is rarely done. Instead, the potential is computed at a set of (often) pre-determined geometries and a multi-dimensional function that depends on fitting parameters is adjusted until it nearly reproduces the computed potential values. This function is the potential energy surface (PES). PESs enable one to do calculations; they also make it possible to think about the motion of atoms in physical terms.

The PES idea is based on the Born-Oppenheimer (BO) approximation. It enables one to solve the full Schrödinger equation by dividing it into an electronic Schrödinger equation and a nuclear Schrödinger equation. There are many electronic structure methods, of varying degrees of accuracy, that can be used to solve the electronic Schrödinger equation. It is now straightforward to compute electronic energies at tens of thousands of points. ${ }^{1-3}$ To fully exploit this ability it is important to develop general and easily used PES fitting methods. In some applications, it is necessary to use more than one PES. It is in principle possible to forgo building PES(s) and instead to solve the electronic Schrödinger equation at all geometries at which a potential value is required. This strategy is often called direct dynamics. ${ }^{4}$ It is sometimes used when classical equations of motion are used to study the motion of the nuclei. Direct dynamics is costly because electronic energies are discarded after they are computed. Such calculations are usually done with cheap electronic structure methods, e.g. some form of DFT (density functional theory) ${ }^{5,6}$. Discarding is clearly wasteful because it means that energies at nearby geometries must be calculated from scratch without exploiting results of previous calculations. Direct dynamics ideas have also been used when using quantum mechanics to $\operatorname{study}^{7-9}$ the motion of the nuclei, but they are efficient 
only if wavefunctions remain localized. If direct dynamics is too costly (as is often the case when one wishes to use accurate electronic structure methods or to do long classical simulations), a PES is imperative. When building a PES, electronic energies are not discarded. In contrast, all the electronic energies are used.

\subsection{Major PES fitting methods}

When building a PES, a key question is how many ab initio points (each corresponding to a geometry) are required. The larger the number of points the costlier it is to make the PES. The required number of points depends on the system and on the fitting method. One general strategy is to guess a fitting function that depends on a small number of parameters. ${ }^{10-14}$ If it is possible by varying the parameters to obtain a PES that does a good job of reproducing ab initio points that cover a range large enough to compute the properties of interest, then this strategy works well. However, it has two key disadvantages: 1) when the PES is not simple, it is difficult to guess an appropriate fitting function or no such function exists; 2) it requires designing a new fitting function for every problem. Articles in this issue are about machine learning (ML) alternatives to guessed fitting functions. ML alternatives are general fitting methods that can be used to fit any PES. They overcome both disadvantages. In this article, we review work on using a particular ML method, neural networks (NN), to fit PESs of small molecules, where high accuracy is required. General fitting functions necessarily require more parameters and more points than would be necessary if it were possible to guess a good fitting function. Because with ML in general and NN in particular fitting functions have more parameters, they also require doing more electronic structure calculations. There are many non-NN methods that might also be considered non-ML methods that use either fitting or interpolation techniques to build PESs. Examples range from splines $^{15}$ to modified Sheppard interpolation (MSI), ${ }^{16-19}$ interpolating moving least squares (IMLS), ${ }^{20-22}$ permutationally invariant polynomial (PIP) bases, ${ }^{23-27}$ reproducing kernel Hilbert space (RKHS) interpolations, ${ }^{28,29}$ distributed approximating functional (DAF) bases, ${ }^{30,31}$ and other approaches. Recently, combinations of different approaches have been shown to be a powerful way to increase the quality of PES construction by simultaneously capitalizing on their different advantages; examples are PIP-IMLS, PIP-NN, and NN-multibody expansion combinations which will be considered below. Some methods (e.g. MSI and IMLS) make a PES by patching together local fits or interpolants, others use combinations of global functions (e.g. PIP). It is also common to fit to a sum of functions that depend on a subset of the full set of coordinates. ${ }^{23,}{ }^{32-36}$ Very 
accurate PESs can be constructed using symmetry adapted perturbation theory. ${ }^{37}$ Excellent surfaces for clusters and bulk systems have been made using the MB-pol approach of Paesani and co-workers. ${ }^{38}$ There are other ML methods ${ }^{39}$ which are finding use in PES construction, including non-parametric approaches like Gaussian process regression (GPR). ${ }^{40-42} \mathrm{NNs}$ will be compared with some of these methods below.

\subsection{Scope of this review}

In this review, we focus on neural network based potential energy surfaces for molecules. We review progress made in neural network based methods for the construction of interatomic potentials from discrete samples (such as ab initio energies) in internal coordinates which are suitable not only for classical molecular dynamics but also, and in particular, for quantum dynamics including quantum reaction dynamics and calculations of (ro-)vibrational spectra. ${ }^{43-45}$ We focus on methods for building molecular PESs that explicitly include all many-body contributions, even though some of the methods we review limit the degree of coupling, due either to a desire to limit computational cost or to limited data. Explicit and direct treatment of all manybody contributions is only practical for sufficiently small molecules, which are therefore the primary focus. Computing a spectrum or some other dynamical property reveals the accuracy of a PES and is therefore a stringent test. Dynamical properties can be computed either from a PES available in the form of a function (e.g. a NN) or by calling a routine at points in configuration space (as in the case of some other ML based methods). In these applications, PESs often need to be built to an accuracy of only a few $\mathrm{cm}^{-1}$, typically corresponding to about $1 / 10,000^{\text {th }}$ of the energy range considered. Getting properties of the PES right is important even for a qualitative description of some properties. For example, if a PES is not invariant with respect to permutations and inversion then the degeneracy pattern of the levels may not be correct. Vibrational spectra are therefore a sensitive probe of such properties. Vibrational spectra of free molecules are measured with sub- $\mathrm{cm}^{-1}$ accuracy even for relatively large polyatomic molecules. Quantitatively accurate PESs need to be accurate to several $\mathrm{cm}^{-1}$ in important regions of the accessible configuration space. We will refer to this as "spectroscopic accuracy" and show in the following that NNs are wellsuited to building spectroscopically accurate PESs. The stringency of the quantum dynamics test is also due to the fact when using a tensor product quadrature grid, points are required in a very large region of configuration space. Such calculations truly test the global quality of the potential function and are sensitive to overfitting, which in the worst case may manifests itself as "holes" in 
the PES, from each many PESs suffer. ${ }^{44,46-49}$ Much of the literature on NN PESs has been on ground state PESs, however there are also papers on NN based excited state and diabatic PESs; some of them will be reviewed below.

We do not consider in detail, but do mention key ideas of NN based PES construction methods based on the decompositions into atomic contributions which are developed in particular for solid state and interfacial systems. These Ansätze work best when the number of atoms is so large that it is too costly to explicitly and directly consider all many-body contributions to the PES. They typically represent a PES as a sum of NNs corresponding to atomic contributions with environment-dependent functions. ${ }^{50-63}$ They are often applied when accuracy requirements are not as stringent, although in principle such methods can provide $\mathrm{cm}^{-1}$-level accuracy for molecules (which is achieved precisely because for a small molecule the component NNs can explicitly model all many-body interactions), these cases are reviewed below. ${ }^{64-66}$ of course when one considers the molecular degrees of freedom of a molecule on a surface, possibly including a limited number of surface atoms, the methods considered here are fully applicable, and we will provide examples thereof in the following.

Neural network, and in general machine learning based approaches are advantageous in high dimensional configuration spaces where simple fitting or interpolation techniques such as splines or polynomial expansions do not work well. Fitting a tensor product representation fails catastrophically in high dimensions. This is often explained by invoking the curse of dimensionality according to which if the sampling density is constant, the number of required data points and functional terms grow exponentially. ${ }^{67} \mathrm{~A}$ key advantage of neural networks is that they are, as will be shown in section 2.1, non-direct product representations. Although the cost of a NN representation generally grows with the dimensionality of the PES, including both the number of required PES samples and of required NN components (neurons), it need not grow exponentially. Nevertheless, the density of sampling of the configuration space is a major issue. Simply put, it is not feasible to sample the configuration space with the density that would be required if one were fitting with a standard polynomial or spline or Fourier expansions to achieve spectroscopic accuracy. 100,000 high quality ab initio data points can be computed at a significant cost even for a small molecule like methane. ${ }^{68}$ On a tensor product grid, this is equivalent to about 3.5 points per degree of freedom (DOF); increasing the number of data points to 1,000,000 would only increase the density of sampling to about 4.7 data points per DOF. For a 7-atom molecule like 
$\mathrm{UF}_{6},{ }^{69}$ increasing the number of data points from 100,000 to $1,000,000$ would increase the density from about 2.2 to only about 2.5 points per DOF. One of the key uses of NNs in PES representation is therefore in methods which aim at PES function reconstruction from very sparse ab initio data. This necessitates going beyond representations with a single NN and towards more complex methods using NNs as building blocks.

This review is organized as follows: in section 2, we will introduce artificial neural networks of the types used to make molecular PESs and describe at a conceptual level (with specific examples provided in subsequent sections) how $\mathrm{NN}$ architecture can be designed to impart

to the PES desired structure and properties. By structure, we mean, for example, expansions in orders of coupling (N-mode representation) or dimensionality reduction or ability to work with sparse data or representations in the sums-of-product (SOP) form. In section 3, we review specific examples of applications of NNs for PES construction for molecules and reactive systems, including PESs for classical and quantum dynamics, for isolated molecules and for molecules on surfaces, and applications of methods beyond standard NNs such as SOP NNs and representations with low-dimensional functions, all conceptually introduced in section 2. In that section, we will also consider the critically important and still underappreciated issue of how representable published PES fitting or test errors are of errors which are important when using a PES to compute dynamical properties. This is related to the fact that the data on which the NN was trained or tested and points at which the PES is required in applications may be rather different. Section 3 also reviews several and still rare cases where NN PESs were compared to other methods on the same systems and same data, as well as some computational aspects. Section 4 contains concluding remarks.

\section{Neural networks for potential energy surfaces}

\subsection{Introduction to neural networks used in PES fitting}

Artificial neural networks (what we will mean by the abbreviation $N N)^{70-72}$ are often presented as model systems mimicking an interconnected network of neurons in a brain. While there is some value in such a presentation, and it is justified for example by the fact that often used sigmoid activation functions can be said to mimic neuron activation, the mathematical NN objects and particular NN types used in PES construction need not invoke references to biology, even as prior 
reviews $^{70,71,73}$ amply cover this analogy. Potential energy surfaces are smooth functions, and the NNs we consider are of the regression rather than classification type. ${ }^{73}$ We believe the operation and utility of NNs in computational chemistry and specifically for PES construction is better understood from the perspective of a non-direct product basis representation. We begin with the simple case of the so-called single-hidden layer NN. In it, one builds an input-output mapping $f(x)$ as

$$
f(\boldsymbol{x})=\sum_{n=0}^{N} c_{n} \sigma\left(\boldsymbol{w}_{\boldsymbol{n}} \boldsymbol{x}+b_{n}\right)
$$

where $f(\boldsymbol{x}), \boldsymbol{x} \in R^{D}$ is assumed to be a smooth (which is true for diabatic PESs and adiabatic PESs in regions excluding conical intersections ${ }^{74}$ ) multivariate function and $\sigma$ is a univariate function (such as the often used sigmoid function) called neuron activation function or simply neuron. The argument of $\sigma$ is a linear form of the coordinates $x \cdot \boldsymbol{w}_{n}$ and $b_{n}$ are tunable parameters which are fitted to reproduce a given set of training data $\left\{f^{(j)}, \boldsymbol{x}^{(j)}\right\}, j=1, \ldots, M$ (Eq. 1 includes the constant term in the choice of $\boldsymbol{w}_{0}$ and $b_{0}$ ). Eq. 1 is often written as $f(\boldsymbol{x})=$ $\sigma\left(\sum_{n=0}^{N} c_{n} \sigma\left(\boldsymbol{w}_{\boldsymbol{n}} \boldsymbol{x}+b_{n}\right)\right)$ i.e. with an output neuron, which, however, can be subsumed in the definition of $f$, without loss of generality.

Eq. 1 has a long history. It goes back to the Kolmogorov theorem ${ }^{75}$ that established the principle of representation of a multivariate function via a superposition of (generally different) univariate functions. It was followed by a series of papers which step by step relaxed the requirements on the function $\sigma$ and offered constructive proofs, ${ }^{76-90}$ first establishing that one and the same monotonic function can be used for all $n$ and relatively recently, in 1998, establishing that that $\sigma$ need only be smooth and non-linear. ${ }^{91}$ In particular, it need not be sigmoid even though the most widely used NNs are those with sigmoid-shaped neurons, ${ }^{77,92}$ most often of the hyperbolic tangent form, $\sigma(x)=\left(e^{x}-e^{-x}\right) /\left(e^{x}+e^{-x}\right)$, although other sigmoid-shapes functions have also found use. The single-hidden layer NN of Eq. 1 is a universal approximator, ${ }^{76,78,83}$ i.e. there is a finite number of neurons $N$ such that for any however small $\delta$, the $L^{2}$ norm $\left|f(\boldsymbol{x})-\sum_{n=0}^{N} c_{n} \sigma\left(\boldsymbol{w}_{\boldsymbol{n}} \boldsymbol{x}+b_{n}\right)\right|<\delta$. The universal approximation theorem says nothing about 
sparsity of samples at which $f(x)$ is known. If data availability is not an issue, there is in principle no need to use multi-layer or so-called deep NNs.

What is important to stress, especially in the context of computational chemistry, is that Eq. 1 is an expansion of $f(x)$ in a non-direct product basis. It is an expansion in a tunable basis with the parameters $\boldsymbol{w}_{n}$ and $b_{n}$. It is this tunability and the non-direct product quality of the basis which enables NNs to obviate the exponential scaling of the cost in principle and, as examples below will show, in applications. Eq. 1 is an example of a ridge $\mathrm{NN},{ }^{77}$ so called because the argument of $\sigma$ defines a plane dividing the space. When the argument of $\sigma$ is expressed as $b_{n}\left|\boldsymbol{w}_{\boldsymbol{n}}-\boldsymbol{x}\right|^{2}$, one obtains a so called radial basis function (RBF) NN. RBF NNs are also universal approximators ${ }^{93}$, 94 and also in principle allow avoiding exponential scaling, specifically, for band-limited functions (i.e. for functions whose Fourier spectrum decays rapidly beyond a certain frequency). ${ }^{95}$

Note that $f$ in Eq. 1 can be replaced with a vector $f$ with components $f_{k}$, in that case the coefficients will also depend on $k, c_{n k}$, while the basis can remain the same. This would be a NN with multiple outputs which could be for example energies and forces or potential values of different states. This is advantageous as forces with many ab initio methods are computed with very little overhead. In the following, for simplicity, we will not carry $k$ in the equations, on the understanding that the output can have multiple components.

The properties of Eq. 1 described above are maintained if $\boldsymbol{x}$ are not the original coordinates (spanning the configuration space of a molecule) but linear or non-linear functions of them:

$$
f(\boldsymbol{x})=\sum_{n=0}^{N} c_{n} \sigma\left(\boldsymbol{w}_{\boldsymbol{n}} \boldsymbol{y}(\boldsymbol{x})+b_{n}\right)
$$

The dimensionality of $\boldsymbol{y}$ need not equal that of $\boldsymbol{x}$. Indeed, augmented inputs (e.g. using squares of elements of $\boldsymbol{x}$ together with $\boldsymbol{x}$ ) is a well-known technique in NN training. ${ }^{72}$ In the following sections, we will highlight the utility of using NNs in transformed coordinates. When $y$ are outputs of another "layer" of neurons, $y_{k}(\boldsymbol{x})=\sum_{n=0}^{N \prime} c^{\prime}{ }_{k, n} \sigma\left(\boldsymbol{w}_{\boldsymbol{k}, \boldsymbol{n}}^{\prime} \boldsymbol{x}+b^{\prime}{ }_{k, n}\right)$, one obtains a two-layer NN. In the general case of several layers, 


$$
f_{l}=\sigma_{\text {out }}\left(\sum_{k_{n}=0}^{N_{n}} w_{l, k_{n}}^{(n)} \sigma_{n, k_{n}}\left(\sum_{k_{n-1}=0}^{N_{n-1}} w_{k_{n}, k_{n-1}}^{(n-1)} \sigma_{n-1, k_{n-1}}\left(\ldots \sum_{k_{1}=0}^{N_{1}} w_{k_{2} k_{1}}^{(2)} \sigma_{1, k_{1}}\left(\sum_{i=0}^{d} w_{k_{1} i}^{(1)} x_{i}\right)\right)\right)\right)
$$

where we kept subscripts on $\sigma$ to highlight the fact that they need not be the same neither from layer to layer nor within the same layer, even though typically the same function is used, with the exception of the output layer where it is common to use linear or piecewise linear activation functions. In Eq. 3, the biases ( $b_{n}$ in Eqs. 1,2$)$ are subsumed into the weights at $x_{0}=1$, for simplicity. Although single-hidden layer NNs are already universal approximators, multilayer NNs are useful when dealing with sparse and very uneven data distributions ${ }^{96}$ and to enable representations with lower-dimensional terms, including dimensionality reduction, as described in the next section.

Neural network fitting involves non-linear optimization of a large number of parameters $w_{k_{n}, k_{n-1}}^{(n-1)}$ to minimize an error measure, typically the rmse (root mean square error) between the $\mathrm{NN}$ output and the target values of the potential (and derivatives, as applicable). The parameters are typically initialized randomly within a certain range (such as [-1,1]). Often, the inputs $\boldsymbol{x}$ and targets $\boldsymbol{f}$ of the $\mathrm{NN}$ are scaled to the same range (usually $[-1,1])$ for fitting. This is done on one hand to approximately match the range of the data to the range of the initialization of the parameters and on the other to simplify the task of the optimizer so that it does not have to work with drastically different length scales in different dimensions and, in the case of a multi-output $\mathrm{NN}$, with drastically different scales of the different outputs which would permit errors in some outputs to dominate the total rmse at the expense of other outputs. The scaling ultimately helps find a good local minimum of the error. A NN fit finds a local rather than global minimum in the parameter space. As parameter initialization is typically random, there is variation in fit outcomes from run to run as the optimization ends generally in different local minima. As long as the rmse variation from run to run is small, this is not a problem. A committee of NNs (i.e. averaging outputs of different NN fits to the same data) can be used to minimize the random component of the variation (see below). ${ }^{97,98}$

Another reason for variation of fit quality from run to run is the typically random split of the total dataset $\left\{f^{(j)}, \boldsymbol{x}^{(j)}\right\}$, into a training (fitting) and a test set, and sometimes into a training, a validation, and a test set. Black-box fitting methods in general and NNs in particular may overfit the data which means that a better match of the fitted $\left\{f^{(j)}, \boldsymbol{x}^{(j)}\right\}$, which can be achieved by simply 
increasing the size of the NN (number of neurons), leads to a poorer PES due to larger errors between the fitting points. We emphasized above the issue of the low density of sampling of multidimensional PESs. Overfitting is a major issue. That is why one typically reserves part of the data for testing purposes. It is the error on that test set which is not used during fitting, rather than on the training set, that is indicative of the global accuracy of the PES. Whenever possible, we will refer to that error in the following. It is important that the test set be representative; we will address this serious issue below. A part of the data is sometimes reserved for a validation test, which is also not used to guide parameter optimization but on which the error is computed at all steps of the optimization. The fitting is stopped when the error on the validation set starts increasing (socalled early stopping). ${ }^{57,99}$

\subsection{Achieving different types of representations via choice of the NN architecture or a combination of NNS}

Single-hidden layer NNs provide a natural and elegant way to achieve a sum of products (SOP) representation of a PES which greatly simplifies calculation of the integrals required to use variational quantum dynamics methods. ${ }^{44}$ In particular, multiconfiguration time-dependent Hartree $(\mathrm{MCTDH})^{43}$ calculations are usually done with a SOP PES. Exploiting a result of Gorban, who showed that the neuron activation function "need only be non-linear, nothing else"91 in order for a NN to possess the universal approximator property, one can use exponential neurons to immediately obtain SOP PESs, as Manzhos and Carrington showed in 2007: ${ }^{100}$

$$
f(\boldsymbol{x})=\sum_{i=0}^{M} w_{i}^{(2)} \prod_{k=0}^{d} e^{w_{i k}^{(1)} x_{k}}
$$

In 2014, Koch and Zhang re-discovered the idea of a SOP NN and proposed using multiplicative artificial neural networks. ${ }^{101}$ They used neurons which are products of erf, i.e. sigmoid, functions which were claimed to possess the universal approximator property ${ }^{102,103}$ although the proofs they reference ultimately did not use product neurons. ${ }^{104,105}$ We will show in examples below that using an exponential neuron NN one can produce a SOP PES with fewer terms and better global accuracy than with other approaches. 
We now consider the multilayer NN of Eq. 3. The numbers of neurons in different layers are generally different. Outputs of any hidden layer can be considered as new, transformed coordinates, in which a NN made of subsequent layers fits the data. Eq. 2 described such a case where $\boldsymbol{y}(\boldsymbol{x})$ are the transformed variables in which a single-hidden layer of Eq. 2 operates. The dimensionality of $\boldsymbol{y}$ can be higher than that of $\boldsymbol{x}$ or lower. If it is lower, dimensionality reduction is performed. When $\boldsymbol{y}(\boldsymbol{x}), \boldsymbol{y} \in R^{d}, d<D$, are outputs of a previous layer of neurons, one obtains what is well-known in signal and image processing literature as an autoencoder. ${ }^{106,107}$ In PES construction, there are two major reasons to employ dimensionality reduction: (i) one may wish to work with redundant coordinates (such as interatomic distances) which expand the dimensionality beyond the intrinsic dimensionality which could complicate fitting, in which case $D$-reduction back to the intrinsic dimensionality can be performed without loss of accuracy; (ii) with a finite density of sampling, it may not be possible to reconstruct the $D$-dimensional function sampled at the data points. Indeed, the dimensionality of a set of point samples in a $D$-dimensional space varies from 0 (for a single point) to $D$ (for an infinitely dense set). Simply put, a finite-size sample in a $D$-dimensional space is not a $D$-dimensional object. When the intrinsic dimensionality of the data is smaller than the space dimensionality $D$, it may be advantageous to reduce the dimensionality to the intrinsic dimensionality $D_{\text {intr }}$ in which fitting may be easier, in particular, overfitting can be reduced due to the higher density of data in the $D_{\text {intr }}$-dimensional space. Examples will be given below.

The same issue of the necessarily low density of sampling in multidimensional spaces justifies the use of NN architectures or NN based methods using expansions in orders of coupling including multi-body or N-mode expansions. ${ }^{10,34,35,108,109}$ Such expansions fall into the general paradigm, introduced by Rabitz and co-workers, of high-dimensional model representations (HDMR). ${ }^{110,111}$ In HDMR, $f(x)$ is represented as

$$
\begin{gathered}
f(\boldsymbol{x})=f_{0}+\sum_{i=1}^{D} f_{i}\left(x_{i}\right)+\sum_{\substack{i=1, j=i+1}}^{D} f_{i j}\left(x_{i}, x_{j}\right)+\cdots+\sum_{i_{1}, i_{2}, \ldots, i_{d}}^{D} f_{i_{1}, i_{2}, \ldots, i_{d}}\left(x_{i_{1}}, x_{i_{2}}, \ldots, x_{i_{d}}\right)+\cdots \\
\quad+f_{1,2, \ldots, D}(\boldsymbol{x})
\end{gathered}
$$


The full HMDR expansion is exact; it is approximate when truncated to an order $d<D$ :

$$
f(\boldsymbol{x}) \approx f_{0}+\sum_{i=1}^{D} f_{i}\left(x_{i}\right)+\sum_{\substack{i=1, j=i+1}}^{D} f_{i j}\left(x_{i}, x_{j}\right)+\cdots+\sum_{i_{1}, i_{2}, \ldots, i_{d}}^{D} f_{i_{1}, i_{2}, \ldots, i_{d}}\left(x_{i_{1}}, x_{i_{2}}, \ldots, x_{i_{d}}\right)
$$

The key advantage of this approximation is that the component functions $f_{i_{1}, i_{2}, \ldots, i_{d}}\left(x_{i_{1}}, x_{i_{2}}, \ldots, x_{i_{d}}\right)$ are of lower dimensionality than $D$. This helps in computing integrals involving the PES, and also reduces the cost of fitting because only the (lower-dimensional) component functions need to be fitted. The $\mathrm{N}$-mode expansion corresponds to defining the component functions on $d$-dimensional hypersurfaces passing through the equilibrium configuration of the molecule (what Rabitz et al. called cut-HMDR ${ }^{110,111}$ ). One disadvantage of the $\mathrm{N}$-mode representation is the need for a separate set of data to fit each component function, with the number of datasets growing combinatorically with $D$ and $d$. It is possible to determine all component functions from a single set of data with a so-called random sampling (RS) HDMR. In the original RS-HDMR, component functions were computed as multi-dimensional integrals ( $D$ - $d$ dimensional for $d$-dimensional component functions), ${ }^{110-113}$ which is a major disadvantage and largely defeats the purpose of simplifying the construction and use of multidimensional functions for which HMDR was introduced in the first place. Both cut-HDMR and RS-HMDR suffer from a combinatorial explosion of the number of terms with dimensionality $D$ and with order of expansion $d$.

Using NNs to fit the component functions makes it possible to realize the real promise of RS-HDMR: all component functions can be fitted simultaneously to one dataset without the need to compute multidimensional integrals. ${ }^{114}$ We note that in Eq. 6, lower-order terms can be subsumed into the terms of dimensionality $d$ without loss of generality:

$$
f(\boldsymbol{x}) \approx \sum_{i_{1}, i_{2}, \ldots, i_{d}}^{D} f_{i_{1}, i_{2}, \ldots, i_{d}}\left(x_{i_{1}}, x_{i_{2}}, \ldots, x_{i_{d}}\right)
$$


which simplifies the form of the representation and the cost of its construction This simplification is achieved trivially with NN component functions but not when evaluating them as integrals in the original RS-HDMR formulation. The number of terms in Eq. 7 still scales combinatorically (as the binomial coefficient $\left(\begin{array}{l}D \\ d\end{array}\right)$, i.e. the number of terms is hard-wired to the values of $D$ and $d$ ). When representing component functions with NNs, it is possible to define - as part of the NN fitting itself - new coordinates in which one can decouple the number of terms and their dimensionality $d$ and obtain any desired accuracy by increasing either or both. ${ }^{115-117}$ This is achieved by using, instead of Eq. 7,

$$
f(\boldsymbol{x}) \approx \sum_{i=i}^{N} f_{i}\left(x_{1}^{(i)}, x_{2}^{(i)}, \ldots, x_{d}^{(i)}\right)
$$

where $\boldsymbol{x}^{(i)}$ are new coordinates which can be obtained by a linear transformation of the original coordinates $\boldsymbol{x}: \boldsymbol{x}^{(i)}=\boldsymbol{A}^{(i)} \boldsymbol{X}+\boldsymbol{b}^{(i)}$. This linear transformation can be performed by a single layer of linear neurons and $\boldsymbol{A}^{(i)}, \boldsymbol{b}^{(i)}$ fitted during the PES fitting. ${ }^{115}$ The total number of new coordinates in all $\boldsymbol{x}^{(i)}$ can be redundant and hence we called this method Red-RS-HDMR-NN. ${ }^{115}$ Avoiding the combinatorial scaling is a major advantage of using NNs. Details and examples will be given in section 3.5. Eq. 7 or 8, or each summand of Eq. 6, can be fit with a single NN by constraining connections between neurons in different layers. This entails minimal modifications of a standard all-connected NN architecture. It can also be realized, for computational efficiency, with individual NNs for each component function. ${ }^{116}$

The approach of Behler and co-workers (often called HDNNP, high-dimensional neural network potential) uses NNs as building blocks in a more complex method. ${ }^{57,118,119}$ The energy is expressed as a sum of atomic contributions which are computed with NNs in an environmentdependent way: ${ }^{50} E=\sum_{i=1}^{N_{a}} N N_{i}\left(G_{i}^{\mu}\right)$, where the NN inputs $G_{i}^{\mu}$ depend on atom type $\mu$ and Cartesian coordinates and local environment of each atom. Symmetry (see also section 2.3) is embedded in the definition of $G_{i}^{\mu}$. The advantage of this representation is extendibility to a large number of atoms which makes it possible to model solids, surfaces, and interfaces, 50, 52, 53, 55-58, 118 , ${ }^{120}$ although the approach of Behler has been used also with small molecules ${ }^{64,65}$ and small 
molecules on surfaces, ${ }^{121-125}$ where the extensivity of the method allows inclusion of many surface degrees of freedom. This is possible because individual NNs are the same for different atoms of the same type (element) and different chemical environments are embedded in $G_{i}^{\mu}$. The approach was later extended from environment-dependent atoms to environment-dependent atom pairs. ${ }^{55}$ The details of the method and its applications, in particular to solids and interfaces with very large numbers of atoms have been well reviewed elsewhere. ${ }^{57,118,119}$ Similar ideas have also been used by Smith et al. ${ }^{62}$ to construct potentials of many relatively large molecules (dozens of atoms) with rmse values on the order of a $\mathrm{kcal} / \mathrm{mol}$ (about $350 \mathrm{~cm}^{-1}$ ). Hu et al. used a similar approach to study solid Si and liquid and solid Na. They wrote the energy as a sum of atomic contributions, but the atomic contributions were computed by decomposing the DFT energy ${ }^{59,60}$ Here we note that these ideas resemble the (modified) embedded atom model ((M)EAM) frequently used for making force fields for metallic systems in that many-body terms are modeled with effective environment dependent functions. ${ }^{126,127}$ Zhang et al. have exploited this similarity further: their Embedded Atom Neural Network (EANN) approach uses, similar to the approach of Behler, a sum of atomic contributions expressed via NNs, but the individual NNs depend, just like in the EAM method, on the density: ${ }^{54} E=\sum_{i=1}^{N a} N N_{i}\left(\boldsymbol{\rho}_{i}\right)$, where components of the vectors $\boldsymbol{\rho}_{i}$ are electron density projections on Gaussian type orbitals of different angular momenta, centered on each atom. In this way, the local atomic environment is captured, and with it many-body contributions to the potential. A mean absolute error (mae) on the order of $5 \mathrm{meV}$ (about $400 \mathrm{~cm}^{-1}$ ) could be obtained when fitting potentials of relatively large molecules (e.g. aspirin, naphthalene, malonaldehyde) to thousands to tens of thousands points with this method. ${ }^{54}$ Ghasemi et al. introduced the charge equilibration via neural network technique (CENT), in which the neural network fits not the energy but the density $\rho$, on which the energy $E$ depends. ${ }^{128}$ The energy is expressed as a

$$
E=\sum_{i=1}^{N_{\text {atoms }}}\left(E_{i}^{0}+\chi_{i} q_{i}+\frac{1}{2} J_{i i} q_{i}^{2}\right)+\frac{1}{2} \iint \frac{\rho(\boldsymbol{r}) \rho\left(\boldsymbol{r}^{\prime}\right)}{\left|\boldsymbol{r}-\boldsymbol{r}^{\prime}\right|} d \boldsymbol{r} d \boldsymbol{r}^{\prime}
$$

where $E_{i}^{0}$ are reference energies, $\chi_{i}$ are environment-dependent atomic electronegativities, $q_{i}$ are atomic charges, and $J_{i i}$ are element-dependent atomic hardnesses. The density is expressed via 
atom-centered spherically symmetric Gaussian functions whose parameters, as well as $\chi_{i}$, are determined during NN training. The method was applied to clusters as well as to solids. ${ }^{128,129}$

Schütt et al. introduced deep tensor neural networks (DTNN), ${ }^{61}$ which are also the basis of the SchNet approach. ${ }^{130}$ In their approach, the molecular structure is encoded in a way similar to the Coulomb matrix ${ }^{131}$ : a vector of nuclear charges $\boldsymbol{Z}$ and a matrix of interatomic distances $\boldsymbol{D}$ are formed ensuring rotational and translational invariance. The distances are expanded in a Gaussian basis, yielding a feature vector $\boldsymbol{d}$, which accounts for the different nature of interactions at various distances. The energy, similar as in the Behler method, is a sum of atomic contributions, $E=\sum E_{i}$. Each atom $i$ is represented by a vector $\boldsymbol{c}_{i}$ which includes a sum of pair-wise interactions, $\sum \boldsymbol{v}_{i j}$, where $\boldsymbol{v}_{i j}$ depend on $\boldsymbol{Z}$ and on interatomic distances via $\boldsymbol{d}$. These interactions themselves are refined during the NN fitting process:

$$
c_{i}^{t+1}=c_{i}^{t}+\sum v_{i j}
$$

where $t$ is the fitting cycle. In this way, the chemical environment of each atom is effectively captured. A key difference from the other NN based methods mentioned above is the use the nuclei charges $\boldsymbol{Z}$. Another difference from standard NNs is the use of the so-called tensor layer of neurons to express $v_{i j}$ :

$$
\boldsymbol{v}_{i j}=\tanh \left[W^{f c}\left(W^{c f} \boldsymbol{c}_{j}+\boldsymbol{b}^{f_{1}}\right) \mathrm{o}\left(W^{d f} \boldsymbol{d}_{i j}+\boldsymbol{b}^{f_{2}}\right)\right]
$$

where o denotes element-wise multiplication, and $\boldsymbol{W}$ and $\boldsymbol{b}$ are parameters. The output of this layer is fed into a standard sigmoid layer to ultimately produce atomic energies $E_{i \cdot}{ }^{61}$ It is important to understand that DTNN is not designed to fit PESs. The goal of DTNN is to produce a computer program that gives the electronic energy and also dipole moment, densities, orbital energies, etc. without solving the electronic Schrödinger equation. The input required by DTNN therefore also requires charges of the nuclei. DTNN is conceptually not just another method for fitting PESs. What DTNN does is conceptually different from fitting ab initio energies as a function of coordinates - it effectively learns the mapping from $Z, D$ to energy and other quantities which are 
typically computed by ab initio calculations. The inclusion of $\mathbf{Z}$ is therefore not a simple addition of a new (kind of) input but is a key element of this new framework. The DTNN can be and has been used to predict multiple molecular properties such as orbital energies or charges, ${ }^{132}$ and it can also be used to fit a PES with very high accuracy. Indeed, sub-kcal/mol accuracy was achieved for energies on a large number of relatively large (dozens of atoms) organic molecules, such as those in the QM9 data set ${ }^{133}$ of about 134,000 small organic molecules made up of $\mathrm{H}, \mathrm{C}, \mathrm{O}, \mathrm{N}$, and $\mathrm{F}$ atoms. ${ }^{61,130,134}$ When comparing the accuracy of a DTNN-type PES with that achieved with other NN based methods, one needs to be mindful of the conceptual difference outlined above.

In the PhysNet approach, ${ }^{135}$ the energy was represented as a sum of atomic contributions and Coulombic interactions:

$$
E=\sum_{i=1}^{N_{\text {atoms }}} E_{i}+k_{e} \sum_{i>j=1}^{N_{\text {atoms }}} \frac{q_{i} q_{j}}{r_{i j}}
$$

where $k_{e}$ is the Coulomb constant, $q_{i, j}$ are partial charges also fitted by the NN, and $r_{i j}$ interatomic distances. The atomic energies, similar to the DTNN scheme, depends on pairwise interatomic distances and nuclei charges. The approach showed an even better performance (energy errors on the order of $0.2 \mathrm{kcal} / \mathrm{mol}$ ) on the QM9 dataset than the DTNN and SchNet schemes. ${ }^{135}$ The Hierarchically Interacting Particle Neural Network (HIP-NN) also uses pairwise interatomic distances and nuclei charges as variables and expresses the energy as a sum of environmentdependent atomic contributions. It achieved an accuracy of about $0.3 \mathrm{kcal} / \mathrm{mol}$ on the QM9 set. ${ }^{136}$ The difference from the above methods is that in HIP-NN the atomic energies $E_{i}$ themselves are further decomposed into contributions over orders $n: E=\sum E_{i}, E_{i}=\sum_{n=0}^{N_{\text {inter }}} E_{i}^{n}$, where $E_{i}^{n}$ represent ( $n+1)$-body contributions, i.e., interactions between atom $i$ and up to $n$ its neighbors. ${ }^{136}$

NN based methods of this type have recently been reviewed in Ref. ${ }^{137}$. As noted in section 1.3, approaches using decompositions into atomic contributions in principle treat many body contributions approximately and indirectly (which does not mean they produce higher errors in applications). Below, we mainly focus on results with methods which treat many-body contributions directly. 
Perhaps the simplest way to use a NN as part of a more involved strategy is a NN committee, ${ }^{97}$ which means simply averaging results of $N_{N N} \mathrm{NN}$ fits to reduce the random component of the error, which will scale as $N_{N N}^{-1 / 2}$. The random component may originate from the randomness of the initialization of $\mathrm{NN}$ weights or from the randomness of training data selection (e.g. if the total dataset is randomly split into the training and test sets). NN committees have been used in multiple works. ${ }^{68,138-147}$. A significant component of the fitting error is, however, non-random. ${ }^{98,141}$

Last but not least, NNs can be used to fit parameters of an empirical PES function. For example, in Ref. ${ }^{148}$, the many-body term of the Tersoff potential ${ }^{149,150}$ for benzene was represented with an NN depending on the bonding environment. In Ref. ${ }^{151}$, the PES of Si 5 cluster was fitted to a Tersoff potential whose nine parameters were made functions of the internal coordinates which were fitted with a neural network. The PES was fitted to about 10,000 DFT points with a rmse of $0.0148 \mathrm{eV}\left(120 \mathrm{~cm}^{-1}\right)$, compared to a NN PES for the same system from the same group with an rmse of $30 \mathrm{~cm}^{-1}$ (albeit fitted to 28,000 points). ${ }^{152}$ The idea of NN-fitting potential parameters has recently been combined with the representation of the potential as a sum environment-dependent atomic contributions. ${ }^{153}$

\subsection{Symmetry, asymptotics and extrapolation}

An important issue in using NNs to build PESs is ensuring symmetry. Even if symmetry errors are small, the degeneracy pattern of ro-vibrational states may be wrong because degenerate levels are wrongly split by the PES. The neural network of Eq. 3 is agnostic of symmetry. Symmetry can be incorporated into a NN based potential by inputting symmetry-adapted coordinates. ${ }^{154-156}$ Symmetry-adapted coordinates can be, for example, functions of interatomic distances. ${ }^{23,68,157}$ Pairwise interatomic distances based coordinates that are used in methods like DTNN, PhysNet, SchNet, HIP-NN and similar satisfy permutational symmetry by construction. ${ }^{61,130,135,136}$ Permutationally invariant polynomials have been successfully used as arguments of the NN. ${ }^{158}$ The number of PIP inputs can, however, be very large, which complicates fitting. Zhang and coworkers introduced so-called fundamental invariants which allow significantly reducing the number of PIP-based inputs. ${ }^{159}$ Fundamental invariants are a set of invariant polynomials that contains the smallest number of invariant polynomials which can generate all the invariant polynomials. ${ }^{159}$ Examples are given in section 3. 
Alternatively, one can use so-called symmetry-adapted neurons ${ }^{160}$ which is equivalent to symmetry adapting the arguments of the neuron activation function. Nguyen and Le introduced a NN layer whose role is to form symmetrical outputs which are then passed to the next hidden layer which effectively performs fitting in symmetry-adapted coordinates. ${ }^{161}$ If a primitive NN PES does not have desired symmetry properties, a NN PES that does have the desired properties can be obtained from the primitive PES by averaging over symmetry-equivalent configurations. ${ }^{68,}$ 139, 156 , 162-164 The primitive PES can be trained on a data set that includes all the symmetrically equivalent points, which are "free" in the sense that no additional ab initio calculations are required. ${ }^{68,115,139-}$ 141, 156, 163, 165-167 This can significantly reduce the required number of ab initio points. ${ }^{163}$ It is often not necessary to fit points in the entire accessible region of configuration space, which might be much larger than the symmetrically unique region. Instead, it is enough to fit a set of points that includes points in the symmetrically unique region and some points beyond its boundaries. ${ }^{68,139 \text {, }}$ 162163 Points can be mapped to a symmetry unique region for example by ordering interatomic distances. ${ }^{68,152,164,168}$ The advantage of averaging instead of only using points in the symmetry unique region is that it allows dealing with cases when a center of symmetry (e.g. minimum geometry) is located at the edge of the region of interest of the configuration space. ${ }^{68,139,162,168}$ Examples and comparisons will be given in section 3.

The treatment of symmetry is complicated for molecules on surfaces, where the symmetry of the surface should also be taken into account. In their NN method which is well suited for solids and interfaces, ${ }^{53,55,56}$ Behler and co-workers introduced symmetry adapted functions to take into account the symmetry of the surface potential. ${ }^{120}$ Symmetry functions are effectively new, symmetry-adapted coordinates on which the NN depends and which use, in particular, trigonometric and exponential functions to account for surface periodicity and the diminishing effect of the surface potential at long range. A similar approach (symmetry adapted coordinates with periodicity handled by the use of trigonometric functions) was also used by others including Lorenz et al., ${ }^{155,169}$ Ludwig and Vlachos, ${ }^{156}$ Jiang and Guo, ${ }^{170,}{ }^{171}$ and Shütt et al. ${ }^{61}$

Neural networks by themselves do not assure correct asymptotic behavior. This has to do with their notoriously bad extrapolation properties. It is a problem common to many black-box methods. A standard and efficient way to deal with this is by choosing coordinates which effectively map the asymptotic region to the sampled part of space. A popular and recommended choice in the NN literature, also used with non-NN fits, is to use transformed interatomic distances 
$R_{i j}$ in the form $\exp \left(-R_{i j} / a\right)^{24,68,139,172-174}$ or $1 / R_{i j} .{ }^{163}$ The symmetry treatment is also simplified in interatomic distances. Similar solutions can be used in bond coordinates for bonds. For angular coordinates, the use of trigonometric functions effectively achieves a mapping into an interpolation region.

In Ref. ${ }^{175}$, Malshe et al. studied NN PES quality for several molecules (HOOH, HONO, $\mathrm{Si}_{5}$, and vinyl bromide) when using different coordinates (NN input vectors). They considered interatomic distances, inverse powers of the interatomic distance, three-body angles, and dihedral angles. Both redundant and nonredundant input vectors were investigated. They found that a Zmatrix ${ }^{176}$ specification of the molecular configuration, similar to that often used in in electronic structure calculations, gave the least accurate $\mathrm{NN}$ for $\mathrm{Si}_{5}$ and vinyl bromide. The reason was related to the discontinuity present in the dihedral angle for planar geometries. The use of trigonometric functions of the angles as input elements produced significantly improved fitting accuracy as this choice eliminates the discontinuity. The most accurate fits were obtained when the elements of the input vector were taken to have the form $R_{i j}^{-n}$. Our experience was somewhat different: we obtained good results with bond coordinates (better than with interatomic distances when both were tried), but we explicitly symmetrized the PES and sampled and fitted across the edge of the symmetry unique region if symmetry centers were on that edge. ${ }^{68,114,139,162,177}$

\section{Applications of neural networks to build potential energy surfaces}

\subsection{Brief timeline of neural network based potential energy surfaces: from a curiosity to spectroscopy accuracy}

Because it was known that any analytic function can be represented arbitrarily accurately with a NN, 25 years ago scientists began using NNs to build a PES from ab initio points. ${ }^{154}$, 169, 178-180 However, until about 15 years ago, NN PESs were considered in the quantum dynamics community as a sort of curiosity due to their mediocre accuracy ${ }^{71,181}$ rather than a robust method of first resort for obtaining spectroscopically accurate potentials. For example, a 1994 paper includes the statement: "For many problems ... an attempt to use neural networks ... will lead to low accuracy and lengthy computations”. ${ }^{181}$ The first NN PES was for CO on a Ni surface. ${ }^{179}$ Many of the important ideas were introduced in that paper. The authors discussed choosing the number of neurons, overfitting, the choice of the fitting points, etc. In 1996, Clary and co-workers 
published NN PESs for four-atom Van der Waals clusters. ${ }^{180}$ The mean error, at test points on their PESs was about $25 \mathrm{~cm}^{-1}$.

The first NN PESs made with two hidden layers were published shortly thereafter. ${ }^{182,} 183$ Prudente and Neto ${ }^{160}$ introduced for the first time the idea of using a symmetrized hidden layer to ensure that the NN PES is invariant with respect to permutation of identical nuclei. Symmetrized input was used by Gassner et al. ${ }^{154}$ and Lorenz et al. ${ }^{169}$ Other authors, ${ }^{184}$ instead, fit only the symmetrically unique region of the PES. Raff and colleagues published a series of papers about NN fits and proposed new ways of choosing fitting points. ${ }^{151,185-189}$ Several authors have used NNs to fit terms in a many-body or N mode representation of a PES. ${ }^{114,151,166,189}$ The idea of averaging several NN fits has been employed by several groups. ${ }^{68,138-141,190}$

In 2006, for the first time, it was shown that it is possible to determine NN PESs for threeand four-atom molecules $\left(\mathrm{H}_{2} \mathrm{O}, \mathrm{HOOH}\right.$, and $\left.\mathrm{H}_{2} \mathrm{CO}\right)$ on which many low-lying vibrational energy levels have errors less than $5 \mathrm{~cm}^{-1}$. For all of these molecules, the global fit accuracy (on the test set) is of the order of $1 \mathrm{~cm}^{-1}$ (corresponding to about 1/10,000th of the considered energy range). This was achieved by first fitting a rough NN PES and then fitting a NN to the difference between energies at fitting points and values on the rough surface. ${ }^{162}$ Although increasing the number of neurons is guaranteed to improve the accuracy at the fitting points to any desired value, with finite density of sampling, this will eventually lead to overfitting. Typically, one monitors errors on a test set. However, in multidimensional spaces, such test sets are often not representative of the global accuracy of the potential as they are too sparse. We found that NN potentials can have holes even when the test set errors were low, by using fitting and test point set sizes of several thousand points. Fitting a rough potential first with a small number of neurons guarantees the absence of holes, and refitting the difference with a larger NN improves the overall accuracy, and any overfitting in regions between points not captured in the test set error is contained and does not lead to holes. This was confirmed by the absence of spurious features in spectra. This was an example of using multiple NNs as building blocks of a more involved method more apt to deal with issues which make potential fitting difficult such as finite sampling density.

Over the course of the following decade, multiple authors developed methods which were not simple (sigmoid) NN fits of the PES but imposed structure on the function representation (such as a multi-body representation) or used non-standard NN architectures (such as multiplicative NNs). Ideas were imported into the NN based PES fitting field which showed good performance, 
such as use of permutationally invariant polynomials to treat symmetry or dimensionality reduction. At present, $\mathrm{NN}$ based methods have evolved into rather complex computational architectures capable of doing much more than fitting a PES. In the following subsections, we will give a representative, albeit non-exhaustive, summary of what has been achieved with different NN based methods when fitting potential energy surfaces for molecules and reactive systems, including molecules on surfaces, for use in classical and quantum dynamics simulations.

\subsection{Neural network PESs for classical and quantum dynamics}

\subsubsection{Neural network potentials for classical dynamics}

\subsubsection{Molecular systems}

Among the earliest works on NN potential energy surfaces is the work of No et al. who fitted a water dimer potential in the rigid monomer approximation. ${ }^{191}$ The PES was fitted to about 3,130 MP2 ${ }^{192}$ energies and the mae was $0.04 \mathrm{kcal} / \mathrm{mol}\left(14 \mathrm{~cm}^{-1}\right)$ for the lowest-energy configurations growing to about $0.15 \mathrm{kcal} / \mathrm{mol}\left(52 \mathrm{~cm}^{-1}\right)$ in weakly-bound regions. Latino et al. refitted with NN committees Lennard-Jones models of several small molecules (with up to 12 atoms), and MD

(molecular dynamics) simulations were performed to test the quality of NN fits. ${ }^{146}$ Nguyen and Le fitted the PESs (energies and gradients) of $\mathrm{H}_{2} \mathrm{O}$ and $\mathrm{ClOOCl}$ with their symmetry-adapted NN method. ${ }^{161}$ The $\mathrm{H}_{2} \mathrm{O}$ PES was fitted to only $282 \mathrm{MP} 2$ points with a test rmse of $0.0103 \mathrm{eV}\left(83 \mathrm{~cm}^{-}\right.$ $\left.{ }^{1}\right)$. The ClOOCl PES was fitted to 1,693 MP2 points with a test rmse $0.0409 \mathrm{eV}\left(330 \mathrm{~cm}^{-1}\right)$. The same authors fitted with the same NN type, but using an improved Levenberg-Marquard ${ }^{193}$ algorithm based optimizer, the PESs of $\mathrm{H}_{2} \mathrm{O}, \mathrm{O}_{3}$ and $\mathrm{ClOOCl}$ in Ref. ${ }^{194}$. Somewhat lower test rmse values were obtained for water and ClOOCl: $6.55 \times 10^{-4} \mathrm{eV}\left(5.3 \mathrm{~cm}^{-1}\right)$ for $\mathrm{H}_{2} \mathrm{O}, 0.035 \mathrm{eV}(282$ $\left.\mathrm{cm}^{-1}\right)$ for $\mathrm{O}_{3}$, and $0.0297 \mathrm{eV}\left(239 \mathrm{~cm}^{-1}\right)$ for $\mathrm{ClOOCl}$ when fitting to 191, 2815, and 1693 points, respectively. Nguyen and Le also used their symmetry-adapted NN to fit the PES of $B_{2} S$ and studied by quasiclassical molecular dynamics on the PES the transformation BBS $\rightarrow$ BSB. ${ }^{195}$ Le et al. fitted the PES of ClOOCl to about 35,000 MP2 data with an rmse of $0.0137 \mathrm{eV}\left(110 \mathrm{~cm}^{-1}\right)$ and performed MD simulations of the NN PES to study different dissociation channels. ${ }^{167}$

Raff and co-workers produced NN PES for several molecules and reactions and conducted classical MD simulations on them. Their NN PESs fitted not only ab initio energies but also gradients. In Ref. ${ }^{152}$ they introduced their method, in which the NN fitting was integrated with classical MD-based sampling of configuration space, which can be initiated on an empirical 
potential, and tested it by fitting the PES of vinyl bromide, including dissociation channels, to 1,400 MP4(SDQ) ${ }^{196}$ data. The rmse was about $8 \mathrm{~cm}^{-1}$ - a high accuracy even by today's standards; this was possible due the relatively limited region of configuration space that was of interest. They also fitted a PES or a $\mathrm{Si}_{5}$ cluster to 28,000 DFT points; the rmse was about $30 \mathrm{~cm}^{-1}$. ${ }^{152}$ Single hidden layer NNs were used. In Ref. ${ }^{186}$, Raff and co-workers studied the PES of $\mathrm{SiO}_{2}$ dissociation into $\mathrm{Si}+\mathrm{O}$ or $\mathrm{Si}+\mathrm{O}_{2}$ with a similar approach. Four potential energy surfaces for states with different multiplicities were constructed. The PESs were fitted to about 7,000 DFT points each. The rmse on the test set of points was $0.078 \mathrm{eV}$ (about $630 \mathrm{~cm}^{-1}$ ). In Refs. ${ }^{197},{ }^{187}$, the reactive PES of vinyl bromide was studied in more detail. About $12,000^{197}$ or $72,000^{187} \mathrm{MP} 4$ (SDQ) points were used to sample a larger region of space; six dissociation channels were considered and branching rations into them computed with MD for different amounts of vibrational excitation. The PES rmse was about $0.06 \mathrm{eV}$ (about $500 \mathrm{~cm}^{-1}$ ). Le and Raff then studied cis/trans, trans/cis isomerizations and $\mathrm{N}-\mathrm{O}$ bond dissociation of HONO using a NN committee. ${ }^{138}$ They fitted about 22,000 MP4(SDQ) points and obtained a mae on the order of $90 \mathrm{~cm}^{-1}$. Raff's group also used a NN committee to fit the dissociative PES of hydrogen peroxide. ${ }^{140}$ The PES was fitted to about 26,000 MP2 points with an rmse of $0.01 \mathrm{eV}$ (about $80 \mathrm{~cm}^{-1}$ ).

Previous works used a NN with multiple outputs to fit both the energies and the forces (the gradient), this has the disadvantage of requiring a larger NN and also a different way of using the PES (by using different NN outputs rather than by differentiating the energy) compared to other force fields. This may complicate integration of the PES into existing codes, for example. In Ref. ${ }^{188}$, Raff and co-workers introduced a new NN scheme in which during the optimization of a singleoutput NN (for energy) deviations from the reference gradient and not only from reference energies are penalized. They tested the scheme by refitting a reference $\mathrm{PES}$ for the $\mathrm{H}+\mathrm{HBr}$ reaction and achieving such a high accuracy $\left(1.2 \mathrm{~cm}^{-1}\right.$ in terms of energy rmse) that trajectories computed on the NN potential showed point-by-point agreement with corresponding trajectories on the analytic reference PES. The same approach was used to fit, with a NN committee, the PES for the $\mathrm{BeH}+\mathrm{H}_{2} \rightarrow \mathrm{BeH}_{2}+\mathrm{H}$ reaction to about 9,600 MP2 points with an rmse of $0.0046 \mathrm{eV}\left(37 \mathrm{~cm}^{-1}\right)$.

The NN PES was used for classical MD simulations. Not only gradients but also second order derivatives can be used to fit a NN PES. This was done in Ref. ${ }^{147}$, where the PES for the hydrogen abstraction reaction from methanol was fitted with a NN committee locally around the transition 
state to UCCSD $(T)^{2}$ data. The deviations from the rate constants computed on NN PESs from those computed with reference couple cluster data were only a couple per cent.

The PES for the reaction $\mathrm{OH}+\left(\tilde{\mathrm{X}}^{3} \Sigma^{-}\right)+\mathrm{H}_{2}\left(\tilde{\mathrm{X}}^{1} \Sigma_{\mathrm{g}}{ }^{+}\right) \rightarrow \mathrm{H}\left({ }^{2} \mathrm{~S}\right)+\mathrm{H}_{2} \mathrm{O}+\left(\tilde{\mathrm{X}}^{2} \mathrm{~B}_{1}\right)$ was investigated with the PIP-NN method ${ }^{158,198}$ in Ref. ${ }^{143}$. The PES was fitted to about 30,000 MRCI ${ }^{199}$ points with an rmse of about $3.0 \mathrm{meV}\left(24 \mathrm{~cm}^{-1}\right)$ with a NN committee. Two potential wells $\left(\mathrm{OH}+\cdots \mathrm{H}_{2}\right.$ and $\mathrm{H}_{2} \mathrm{O}+\cdots \mathrm{H}$ ) connected by a submerged saddle point were found on this PES. The PES was used for quasiclassical trajectory studies to compute, in particular, rate coefficients. A committee of PIP-NNs was also used by the same authors to fit a reactive PES for a five-atom system, $\mathrm{H}_{2} \mathrm{O}^{+}+$ $\mathrm{H}_{2} \rightarrow \mathrm{H}_{3} \mathrm{O}^{+}+\mathrm{H}^{200}$ The PES was fitted to 81,000 UCCSD(T) points with an rmse of $1.8 \mathrm{meV}$ (15 $\mathrm{cm}^{-1}$ ) and used for quasiclassical trajectory studies of the rate coefficient and kinetic isotopic effect. The PIP-NN method was used to produce the PES for the reaction $\mathrm{OH}+\mathrm{HO}_{2} \rightarrow \mathrm{H}_{2} \mathrm{O}+\mathrm{O}_{2}$ in Ref. 201. The PES was fitted with a two-hidden later PIP-NN to 108,000 UCCSD(T) energies with an rmse of $12.6 \mathrm{meV}\left(102 \mathrm{~cm}^{-1}\right)$ and quasiclassical trajectory calculations performed on it.

Zhang and co-workers used the fundamental invariants (FI) NN approach ${ }^{159}$ to fit the PES for the reactions $\mathrm{H}+\mathrm{H}_{2} \mathrm{O}_{2} \rightarrow \mathrm{HO}_{2}+\mathrm{H}_{2}$ and $\mathrm{H}+\mathrm{H}_{2} \mathrm{O}_{2} \rightarrow \mathrm{H}_{2} \mathrm{O}+\mathrm{OH}^{202}$ The PES was fitted with a two-hidden layer NN to about 114,000 UCCSD(T) energies with an rmse of $5.7 \mathrm{meV}\left(46 \mathrm{~cm}^{-1}\right)$ as a function of 26 FIs. Quasiclassical trajectory dynamics ${ }^{202}$ and ring polymer molecular dynamics $^{203,204}$ were performed on the PES.

The method of Behler ${ }^{50,57,120}$ was also applied to molecules and clusters: water dimers in Ref. ${ }^{64}$, water dimers and trimers in Ref. ${ }^{66}$ protonated water clusters in Ref. ${ }^{65}$, and to the methanol molecule and $\mathrm{Cu}_{3}$ clusters in Ref. ${ }^{55}$. The water dimer potential was fitted to DFT calculations, with the dataset containing about 5,600 monomer and about 12,000 dimer structures. The rmse was about $1 \mathrm{meV}\left(8 \mathrm{~cm}^{-1}\right)$ per molecule and relative energies of various stationary points on the PES were well reproduced (to $1 \mathrm{meV}$ or better) as well as their vibrational frequencies (to a few $\left.\mathrm{cm}^{-1}\right) .{ }^{64} \mathrm{MD}$ simulations were also performed on this PES. Water trimers were considered in a comparative study comparing with several other methods in Ref. ${ }^{66}$ (see section 3.8 for details). The energies of protonated water clusters as large as $\mathrm{H}_{17} \mathrm{O}_{8}{ }^{+}\left(\mathrm{H}_{2(\mathrm{n}+1)} \mathrm{O}_{\mathrm{n}}{ }^{+}\right.$for $n=1, \ldots$, 8, for a total of about 30,000 structures) were fitted in Ref. ${ }^{65}$ to DFT calculations. Rmse values on the order of $1 \mathrm{meV}$ per atom were obtained. Binding energies of the clusters were accurate to about $1 \mathrm{meV}$ per 
molecule. Protonated water cluster $\left(\mathrm{H}_{2(\mathrm{n}+1)} \mathrm{O}_{\mathrm{n}}{ }^{+}\right.$for $\left.n=1, \ldots, 4\right)$ potentials were also fitted with the Behler method to CCSD(T) energies in Ref. ${ }^{205}$ with an automated point selection scheme (see section 3.7). The NNs were trained on 55,000 energies to rmse values on the order of $0.06 \mathrm{~kJ} / \mathrm{mol}$ $\left(5 \mathrm{~cm}^{-1}\right)$ per atom; binding energies and stationary points had a similar level of error.

The implementation of an NN method based on atom pairs was presented in Ref. ${ }^{55}$, and the PESs for a methanol molecule and the $\mathrm{Cu}_{3}$ cluster were modelled with it. The methanol PES was sampled with molecular mechanics (about 45,000 MM3 ${ }^{206}$ molecular mechanics points) and fitted wtih an rmse of $0.025 \mathrm{meV}$ per atom, while the $\mathrm{Cu}_{3}$ potential was fitted to about 4,000 DFT data with an rmse of about $0.4 \mathrm{meV}$ per atom.

A Behler-type NN was used in Ref. ${ }^{62}$ to fit the potentials of $\mathrm{C}_{10} \mathrm{H}_{20}$, retinol and several other molecules. They showed, in particular, that the NN fitted to DFT can provide better structures and relative energies that semi-empirical methods like AM1, ${ }^{207} \mathrm{PM6}^{208}$ or DFTB ${ }^{201}$. Gastegger and Marquetand applied a Behler type NN to fit the PES for an organic reaction, the Claisen rearrangement of allyl vinyl ether to 4-pentenal to DFT data with an accuracy of around 0.1 $\mathrm{kcal} / \mathrm{mol}\left(35 \mathrm{~cm}^{-1}\right){ }^{209} \mathrm{~A}$ different method also representing the PES as a sum of environmentdependent subsystem contributions was proposed by Sanville et al. ${ }^{210}$ They considered all fiveatom chains within the system, and the total energy is a computed as a sum of NNs each depending on bonds and angles of the chain. In this way, the effect of local chemical environment is captured; at the same time, similar to the method of Behler, extensivity to large systems is obtained. Each $\mathrm{NN}$ input vector (based on five atoms) is associated with its own individual first hidden layer, and the second hidden layer takes its inputs from the outputs of all such partial NNs. They tested the method, in particular on small Si clusters $\left(\mathrm{Si}_{\mathrm{n}}, n=2,3,4\right)$ and obtained a good match with DFT data.

The Behler NN method has also been used to fit the PES around the dividing surface for accurate variational transition state theory calculations for several reactions by Chen and Goldsmith. ${ }^{211}$ An initial NN PES was fitted, the dividing surface computed, and then iteratively refitted until convergence with new data added at and around the dividing surface.

Law et al. used a single-hidden layer NN to fit the PES of $\mathrm{Si}_{2} \mathrm{H}_{2} .{ }^{157}$ The PES was fitted to about 33,000 $\operatorname{CCSD(T)~data;~the~rmse~in~the~energy~region~under~} 25,00 \mathrm{~cm}^{-1}$ above the minimum was $7.2 \mathrm{~cm}^{-1}$. Energies of different isomers and vibrational frequencies were in good agreement with reference data. In Ref. ${ }^{212}$, the Diels-Alder reaction was studied with by means of multisurface 
adiabatic reactive molecular dynamics on potential energy surfaces fitted with NNs as functions of coordinates and nuclei charges, Eq. (12). Training data included 200,000 DFT structures. An rmse of $0.25 \mathrm{kcal} / \mathrm{mol}\left(87 \mathrm{~cm}^{-1}\right)$ was obtained. Use of nuclei charges (atomic numbers) together with coordinates in the form of interatomic distances is a powerful general type of $\mathrm{NN}$ input that works well for different molecules. It is used in particular in the deep tensor neural network (DTNN) approach, ${ }^{61}$ with which it is now possible to fit potential energies of conformers of thousands of molecules in a single project. ${ }^{134}$

\subsubsection{Molecules on surfaces}

Blank et al. pioneered the use of neural networks for the construction of PESs for molecule-surface interactions, critically important in particular in heterogeneous catalysis. ${ }^{179}$ They refitted an existing potential for $\mathrm{CO}$ adsorption of $\mathrm{Ni}(111)$ considering three degrees of freedom to test the method, and produced a DFT based PES for $\mathrm{H}_{2}$ on $\mathrm{Si}(100)$ in 12 dimension s (i.e. including some surface degrees of freedom) from only 750 DFT energies. They achieved a test set error of 0.022 $\mathrm{kcal} / \mathrm{mol}$ (about $8 \mathrm{~cm}^{-1}$ ) when refitting the 3D CO/Ni(111) PES; the mae error on the DFT-based potential for $\mathrm{H}_{2}$ on $\mathrm{Si}(100)$ was $2.2 \mathrm{kcal} / \mathrm{mol}$ (about $770 \mathrm{~cm}^{-1}$ ) or $1.5 \%$ of the energy range. They used rather small single-hidden layer NNs with only about a dozen neurons in the hidden layer.

Lorenz et al. made a NN PES for $\mathrm{H}_{2}$ interaction with potassium-covered $\mathrm{Pd}(100)$ surface in eight dimensions and computed the sticking probability vs impact kinetic energy with MD. ${ }^{169}$ The PES was fitted to 659 DFT energies (of which 40 were reserved for the test set). The test rmse was $74 \mathrm{meV}$ (about $600 \mathrm{~cm}^{-1}$ ). They later refitted an analytic PES of $\mathrm{H}_{2}$ over clean and sulfurcovered $\operatorname{Pd}(100) .{ }^{155}$ The test error when fitting the PESs to about 1,200 points was about $0.1 \mathrm{eV}$ (about $800 \mathrm{~cm}^{-1}$ ).

Behler and co-workers built a NN PES for $\mathrm{O}_{2}$ dissociation on $\mathrm{Al}(111)$ and performed an MD simulation of the reaction. ${ }^{120}$ In the frozen surface approximation, the PES was sixdimensional, and the PES mae on the test set was $0.07 \mathrm{eV}$ (about $565 \mathrm{~cm}^{-1}$ ) after fitting about 3,700 DFT points; about 100 points were used as a test set. They showed a significant reduction in the error due to the use of symmetry adapted functions. Boes and Kitching used a Behler type NN to study oxygen interaction with $\mathrm{Pb}(111) .{ }^{213}$ The PES, including some Pd degrees of freedom, was fitted to about 12,000 DFT points to an rmse of $9 \mathrm{meV}$ per atom.

Ludwig and Vlachos built a six-dimensional PES for $\mathrm{H}_{2}$ dissociation on $\mathrm{Pt}(111)$, in the frozen surface approximation and computed reaction probability as a function of collision 
energy. ${ }^{156}$ They used MD to dynamically improve the training set by adding points where the NN was most inaccurate. With 3,860 configurations computed with DFT they achieved rmse of the training data of $17 \mathrm{meV}\left(137 \mathrm{~cm}^{-1}\right)$ and converged the sticking coefficient.

The PIP-NN method of Guo et al. was also applied to molecules on surfaces. In Ref. ${ }^{171}$, they used the method to refit an analytic PES for $\mathrm{H}_{2}$ on $\mathrm{Cu}(111)$. Different choices of PIPs were studied and rmse values as low as $0.031 \mathrm{eV}\left(250 \mathrm{~cm}^{-1}\right)$ were obtained. In the same work, the PIPNN method was used to fit about 3,400 DFT data for $\mathrm{H}_{2}$ on $\mathrm{Ag}(111)$, and an rmse of $2.6 \mathrm{meV}$ was obtained $\left(21 \mathrm{~cm}^{-1}\right)$. Dissociation probabilities were computed from quasi-classical MD trajectories on these PESs.

3.2.2 Neural network potentials for quantum dynamics and vibrational spectroscopy

\subsubsection{Molecular systems}

Bittencourt et al. fitted PESs of the $\mathrm{A}^{2} \Sigma^{+}$and $\mathrm{X}^{2} \Pi$ states of the $\mathrm{OH}$ molecule and the transition dipole moment function for transition between these states. ${ }^{214}$ They found no advantages of using two hidden layers over a single hidden layer NN. They also explored different neuron activation functions. The NN functions were tested by computing the vibrational levels, the transition probabilities between the two electronic states, and the radiative lifetimes. In particular, ground state vibrational frequencies were within $3 \mathrm{~cm}^{-1}$ of those computed on the extended Rydberg function approximation (ERF) ${ }^{10}$ to the potential. Both approximations (NN and ERF) resulted in frequencies differing from the experimental frequencies by dozens $\mathrm{cm}^{-1}$.

Manzhos, Carrington, and co-workers produced NN PESs for several small molecules and used them to compute vibrational spectra. ${ }^{68,98,100,114-117,139,162,163,165,177,215}$ Ref. ${ }^{162}$ was the first demonstration that neural networks can provide spectroscopically accurate potential energy surfaces. Accurate analytic PESs of $\mathrm{H}_{2} \mathrm{O}, \mathrm{HOOH}$, and $\mathrm{H}_{2} \mathrm{CO}$ were fitted with two-stage NNs, whereby a cruder fit was obtained with a smaller NN which minimized the danger of "holes", and the difference between the crude NN outputs and target potential values was fitted with a larger NN. Any overfitting was mitigated by using a sigmoid or saturated linear output neuron in the larger NN. Test set rmse values of 1, 2, and $2 \mathrm{~cm}^{-1}$ were obtained for $\mathrm{H}_{2} \mathrm{O}, \mathrm{HOOH}$, and $\mathrm{H}_{2} \mathrm{CO}$, respectively, by fitting 1,500, 5,000, and 2,500 symmetry unique points sampled in spectroscopically relevant parts of the potential using Eq. 7 (see section 3.7). The PESs were tested 
by computing vibrational spectra with a highly accurate variational approach using large quadrature grids, thus thoroughly testing the global quality of the potential. For all three molecules, most of the low-lying levels are within $1 \mathrm{~cm}^{-1}$ of the exact results (obtained on reference analytic PESs). In subsequent works, Manzhos and Carrington showed that NNs can be used to represent the PES as an expansion of orders of coupling, ${ }^{114-116,}, 177$ to easily make a sum-of-product form, ${ }^{100}$ and for dimensionality reduction, ${ }^{163,165}$ as described in sections 3.3, 3.5, and 3.6 below. They and co-authors also performed the first controlled comparisons of PES quality and vibrational spectrum quality when fitting the PES with a NN and with other popular methods such as PIP, PIP-NN, PIPIMLS, ${ }^{68}$ and Gaussian process regression, ${ }^{215}$ see section 3.8 for details.

In Ref. ${ }^{139}$, the PES of the phosgene molecule was fitted with a NN committee to 25,000 $\operatorname{CCSD}(\mathrm{T})$ energies with an rmse of $1.0 \mathrm{~cm}^{-1}$. The vibrational spectrum was computed with a collocation method on a Smolyak grid ${ }^{216}$ on the PES.

The group of Hua Guo developed the permutationally-invariant polynomial neural network (PIP-NN) approach. ${ }^{158,198}$ The idea is to use the PIPs proposed by Braams and Bowman, ${ }^{23}$ which take care of the symmetry, as NN inputs. One can select appropriate (typically low-order) PIP inputs. In Ref. ${ }^{158}$, the method was tested by refitting analytic PESs for $\mathrm{H}+\mathrm{H}_{2}$ and $\mathrm{Cl}+\mathrm{H}_{2}$ systems and performing quantum reactive scattering calculations of reaction probabilities. The PESs were fitted to about 1,000 and 2,600 points for $\mathrm{H}+\mathrm{H}_{2}$ and $\mathrm{Cl}+\mathrm{H}_{2}$, respectively. Both PESs were fitted with an rmse on the order of $4 \mathrm{meV}\left(32 \mathrm{~cm}^{-1}\right)$. In Ref. ${ }^{142}$, the method was applied to the $\mathrm{F}+\mathrm{H}_{2} \mathrm{O}$ $\rightarrow \mathrm{HF}+\mathrm{OH}, \mathrm{O}+\mathrm{H}_{2} \mathrm{O} \rightarrow \mathrm{OH}+\mathrm{OH}$, and $\mathrm{H}+\mathrm{H}_{2} \mathrm{O} \rightarrow \mathrm{H}_{2}+\mathrm{OH}$ reactions, and quantum reactive scattering calculations of reaction probabilities were performed on the resulting PESs. About 33,000 MRCI points were used to fit the $\mathrm{F}+\mathrm{H}_{2} \mathrm{O} \rightarrow \mathrm{HF}+\mathrm{OH}$ PES with a test set rmse on the order of $10 \mathrm{meV}\left(81 \mathrm{~cm}^{-1}\right)$. About 34,000 UCCSD(T) points were used to fit the $\mathrm{O}+\mathrm{H}_{2} \mathrm{O} \rightarrow \mathrm{OH}$ + OH PES with a test set rmse on the order of $2 \mathrm{meV}\left(16 \mathrm{~cm}^{-1}\right)$. About 32,000 UCCSD(T) points were used to fit the $\mathrm{H}+\mathrm{H}_{2} \mathrm{O} \rightarrow \mathrm{H}_{2}+\mathrm{OH}$ PES with a test set rmse on the order of $3 \mathrm{meV}\left(24 \mathrm{~cm}^{-}\right.$ ${ }^{1}$ ). They used a committee of three NNs which reduced the overall rmse for these PESs to $6.8 \mathrm{meV}$, $0.8 \mathrm{meV}$, and $1.4 \mathrm{meV}$, respectively. They showed that for systems with more than three atoms, the number of symmetry functions in the NN input vector needs to be larger than the number of internal coordinates and include both the primary and secondary invariant polynomials (the size 
of some input vectors for four-atom systems in that work reached 32). A PIP-NN for the $\mathrm{OH}+\mathrm{CO}$ $\rightarrow \mathrm{H}+\mathrm{CO}_{2}$ reaction was built and full-dimensional quantum and quasi-classical trajectory studies were performed in Ref. ${ }^{217}$, including calculations of reaction probabilities, product translational and vibrational energy distributions, and fundamental frequencies. The PES was fitted to about $74,000 \mathrm{CCSD}(\mathrm{T})$ data and had an rmse of $5 \mathrm{meV}\left(40 \mathrm{~cm}^{-1}\right)$.

Zhang and co-workers produced a series of reactive NN PESs for quantum dynamics. ${ }^{144}$, 145, 168, 203, 218 In Ref. ${ }^{168}$, a NN PES for the $\mathrm{H}_{2}+\mathrm{OH} \leftrightarrow \mathrm{H}_{2} \mathrm{O}+\mathrm{H}$ reaction was fitted to about 17,000 $\mathrm{UCCSD}(\mathrm{T})$ energies, and wavepacket calculations were performed with it to compute reaction probabilities. To speed up NN training, they divided the data points into several parts according to their location (entrance valley, interaction region, product valley), and trained and tested these parts separately. Those segmental parts were then connected with smooth switching functions to yield a global PES. This was compared to fitting one global PES. Two-hidden later NNs were used, and rmse values of about $1.5 \mathrm{meV}\left(12 \mathrm{~cm}^{-1}\right)$ were obtained with both approaches. A NN PES for the reaction $\mathrm{OH}+\mathrm{CO} \rightarrow \mathrm{H}+\mathrm{CO}_{2}$ was fitted in Ref. ${ }^{144}$ to $74,400 \mathrm{UCCSD}(\mathrm{T})$ energies. A committee of six two-hidden layer NNs was used with an rmse of $9.2 \mathrm{meV}\left(74 \mathrm{~cm}^{-1}\right)$ and rmse values close to $1 \mathrm{meV}$ in low-energy regions. Reaction probabilities and complex-formation probabilities were computed with quantum dynamics. A 15-dimensional PES of a six-atom system, the reaction $\mathrm{H}_{2}+\mathrm{CH}_{3} \rightarrow \mathrm{H}+\mathrm{CH}_{4}$, was studied in Ref. ${ }^{164}$. It was fitted to about 48,000 UCCSD(T) energies with a committee of NNs with an rmse on the order of $4 \mathrm{meV}\left(32 \mathrm{~cm}^{-1}\right)$. The PES was later used to study the effect of vibrational excitation on reaction probability with wavepacket dynamics in seven- and eight-dimensional approximations restricting the degrees of freedom of the $\mathrm{CH}_{3}$ moiety. ${ }^{218}$

In Ref. ${ }^{159}$, Zhang and co-workers introduced fundamental invariants (FI) which allow reducing the number of PIPs in the PIP-NN ${ }^{198}$ method. With their FI-NN method, they fitted PES for $\mathrm{OH}_{3}$ and $\mathrm{CH}_{4}$. The $\mathrm{OH}_{3}$ PES was fitted to about 17,000 UCCSD(T) data with a committee of two-hidden layer NNs with an rmse of $1.18 \mathrm{meV}\left(9.5 \mathrm{~cm}^{-1}\right)$, as a function of nine FIs. Reaction probabilities of the $\mathrm{H}_{2}+\mathrm{O}_{2}$ and $\mathrm{H}+\mathrm{H}_{2} \mathrm{O}$ reactions were calculated with full-dimensional quantum scattering on this PES. The $\mathrm{CH}_{4}$ PES was fitted to about 15,000 UCCSD(T) data with a committee of two-hidden layer $\mathrm{NN}$ to an rmse of about $1 \mathrm{~cm}^{-1}$, as a function of 31 FIs. Vibrational energy 
levels were computed with a nine-dimensional quantum mechanical method. ${ }^{159}$ Recently the FINN approach was used to make a PES for the $\mathrm{H}_{2}+\mathrm{HS} \rightarrow \mathrm{H}_{2} \mathrm{~S}+\mathrm{H}$ reaction. ${ }^{145}$ The PES was fitted with a committee of two-hidden layer NNs to about 42,000 UCCSD(T) points with an rmse of 3.1 $\mathrm{meV}\left(25 \mathrm{~cm}^{-1}\right)$. The PES was used in time-dependent wavepacket simulations to compute differential cross sections for the reaction. Lu and Li showed that the number of PIPs can be reduced by using only non-redundant PIPs (NRPIP-NN). ${ }^{219}$ They fitted the PES for the $\mathrm{H}+\mathrm{H}_{2} \mathrm{~S}$ system to about 34,000 UCCSD(T) points with only 9 non-redundant PIPs to a test set rmse of $0.35 \mathrm{kcal} / \mathrm{mol}\left(122 \mathrm{~cm}^{-1}\right)$, which was moderately higher than the test set rmse values of 0.16 $\mathrm{kcal} / \mathrm{mol}\left(56 \mathrm{~cm}^{-1}\right)$ obtained by using 50 4th-order PIPs and of $0.11 \mathrm{kcal} / \mathrm{mol}\left(38 \mathrm{~cm}^{-1}\right)$ by using 22 3rd-order PIPs. However, tunneling corrected transition state theory and quasi-classical trajectory calculations performed on the three PESs resulted in similar quality of observables.

Song et al. refitted an existing PES for $\mathrm{H}+\mathrm{H}_{2}$ and studied the dependence of the quality of the PES on the number of training data points and proposed a relation between optimal NN size and number of data points. ${ }^{220}$ They performed MCTDH calculation on different NN PESs and found little difference in reaction probabilities computed with two-hidden later NN PESs fitted to training data sets whose sizes ranged from about 1,400 to about 3,800 points with rmse values ranging from 4.3 micro-Hartree $\left(0.9 \mathrm{~cm}^{-1}\right)$ to $0.19 \mathrm{mHa}\left(42 \mathrm{~cm}^{-1}\right)$. Their work provided a preview into the issue, discussed in section 3.8, of the different distributions of the points (including test data) used in NN fitting and points at which the PES may be called in applications: they also monitored the rmse on a different dataset, randomly sampled in the whole configuration space, which was on the order of $3 \mathrm{mHa}\left(660 \mathrm{~cm}^{-1}\right)$ for all NNs.

\subsubsection{Molecules on surfaces}

Jiang and Guo built a six-dimensional PES for $\mathrm{H}_{2}$ on $\mathrm{Ag}(111)$ with the permutation invariant polynomial-neural network (PIP-NN) method from about 4,000 DFT energies and computed $\mathrm{H}_{2}$

and $\mathrm{D}_{2}$ dissociative sticking probabilities with quantum dynamics. ${ }^{170}$ Classical MD was used to explore the initial PES and add training data in problematic regions. A two-hidden layer NN was used and the rmse on test set and validation points was about $5 \mathrm{meV}$ (about $40 \mathrm{~cm}^{-1}$ ).

Shen et al. built a fourteen-dimensional potential energy surface for $\mathrm{CH}_{4}$ dissociation on $\mathrm{Ni}(111)$, including selected molecular and surface degrees of freedom. It was fitted to $10^{5} \mathrm{ab}$ initio energies. ${ }^{221}$ Eight-dimensional quantum dynamics simulations were performed to compute a 
sticking probability. A multilayer NN was used; they reported PES fitting rmse values of $5.4 \mathrm{meV}$ $\left(44 \mathrm{~cm}^{-1}\right)$ in the entrance region and $14.4 \mathrm{eV}\left(116 \mathrm{~cm}^{-1}\right)$ in the interaction region. The same group also produced a 15-dimensional fit of the PES for this reaction, for a rigid surface and with full consideration of molecular DOFs, to close to 200,000 DFT energies. ${ }^{222}$ Rmse values of $10.11 \mathrm{meV}$ $\left(82 \mathrm{~cm}^{-1}\right)$ in the entrance region and $17.00 \mathrm{eV}\left(137 \mathrm{~cm}^{-1}\right)$ in the interaction and product regions were obtained.

Liu et al. reported a six-dimensional NN PES for $\mathrm{HCl}$ dissociation on a rigid $\mathrm{Ag}(111)$ surface, on which the reaction was studied with quantum dynamics. ${ }^{223}$ The PES was fitted to about 27,000 DFT energies with a multilayer NN, achieving an rmse of about $5 \mathrm{meV}\left(40 \mathrm{~cm}^{-1}\right)$. The same group later studied the PES for the same reaction fitted with a similar approach to data obtained with different DFT functionals ${ }^{224}$ as well as for $\mathrm{HCl}$ reaction with rigid $\mathrm{Ag}(100)$ and $\mathrm{Ag}(110)$ surfaces. $^{225}$

\subsection{SOP NN}

The most general and popular way of computing wavefunctions is to use a basis set. A systematic and easy approach uses a tensor product basis, each of whose functions is a product of univariate functions. The number of variables is the number of vibrational coordinates. Unless one uses collocation, a basis set approach requires either computing the elements of a matrix representing the potential in the basis or evaluating matrix-vector products with that matrix. Both are facilitated by using a PES that is a sum of products (SOP). When the basis is a tensor product basis, each of the many dimensional potential integrals is a product of 1-D integrals. The SOP PES therefore obviates the need to do multi-dimensional quadratures. It is exploited in multiconfiguration timedependent Hartree (MCTDH) ${ }^{43,226,227}$ and other tensor methods. ${ }^{228-232}$

A PES is a SOP only if the fitting function used to obtain it is a SOP. Most fitting methods are designed for generality and accuracy and do not yield SOP PESs. To use a non-SOP PES with a tensor-based method that requires the SOP form, one converts the PES to SOP form. There are many ways to do this, conceptually the simplest is a Taylor expansion. Although there are other options, ${ }^{233,234}$ POTFIT $^{235}$ is the most popular refitting tool. It is identical to what mathematicians call high order singular value decomposition. ${ }^{236}$ In 2006, we proposed a NN method, called EXPNN (Eq. 4), that can be used either to refit PESs into SOP form or to directly fit a SOP function

to ab initio points. An important advantage of EXPNN is it produces a PES in CP format and not 
in Tucker format. ${ }^{237} \mathrm{CP}$ is an abbreviation for CANDECOMP/PARAFAC ${ }^{237}$ In CP format, there is no link between the factors in different terms. In Tucker format, ${ }^{237}$ the PES is represented in a (carefully chosen) tensor product basis. In general, it is tricky to force a function into CP format. EXPNN is one way of doing it. Schröder has recently introduced new ideas for making CP PESs. ${ }^{238}$ An important advantage of EXPNN is that its memory cost is low. It can be used for molecular systems for which a tensor product grid would be so large that the potential could not be stored on the grid. It is possible, by introducing approximations, to devise versions of POTFIT that do not require storing the potential on a full tensor product grid. 239,240

Using a NN, it is surprising simple to make a SOP PES. All that is necessary to obtain a SOP PES from a NN is to use as activation functions exponential functions (see Eq. 4). Almost all $\mathrm{NN}$ fits are done with sigmoid activation functions, but in principle the activation function need only be nonlinear. ${ }^{91}$ In a NN with a single hidden layer, the number of terms in the SOP PES is equal to the number of nodes in the hidden layer. In general, we have found that using exponential activation functions does not significantly increase the number of required neurons and that the number of terms in the SOP PES is reasonable. ${ }^{100}$ The first SOP NN PESs were reported in Ref. ${ }^{100}$, where Manzhos and Carrington first tested EXPNN on known PESs. Of course it is important that it be possible to achieve small errors, but EXPNN will only be valuable if, in addition, the number of terms in the SOP is small. When refitting the PES of $\mathrm{HOOH}$, the decrease in the test set rmse with the number of neurons was similar for exponential and sigmoid neurons, but was somewhat higher, e.g. with 40 neurons, test rmse was close to $50 \mathrm{~cm}^{-1}$ with both types of $\mathrm{NN}$ and with 90 neurons, the test rmse was about $10 \mathrm{~cm}^{-1}$ with a sigmoid $\mathrm{NN}$ and about $20 \mathrm{~cm}^{-1}$ with EXPNN. They also refitted the NOCl potential with both POTFIT and EXPNN: For the 20/20 (400 contracted terms) POTFIT potential using a set of 168,960 test points, a test set rmse of $60 \mathrm{meV}$ (484 $\mathrm{cm}^{-1}$ ) was obtained (the fit set error can be made arbitrarily small with POTFIT). To achieve the same test-point rmse with EXPNN, only seven terms were needed. ${ }^{100}$

POTFIT is interpolating a PES using a product grid; the POTFIT PES is not only a SOP, it is also a tensor product, more precisely it is in Tucker format. ${ }^{237}$ In contrast, the EXPNN PES is in CP format. ${ }^{237}$ In both the Tucker and the CP format each term in the SOP is a product of factors. In Tucker format, all the terms are products of the same univariate functions, and in CP format, the factors in each term are different. CP format is therefore more flexible, and this flexibility makes it possible to reduce the number of terms in the SOP. It is notoriously difficult to force a 
tensor into CP format. ${ }^{241} \mathrm{~A}$ modified version of EXPNN was also used to make a SOP PES for the multichannel dissociation of vinyl bromide. The PES was written as a sum of lower dimensional terms and the component functions of these terms were represented with EXPNN. The exponential NN test set error of $866 \mathrm{~cm}^{-1}$ was slightly larger than the sigmoid NN error of $769 \mathrm{~cm}^{-1}$; ${ }^{177}$ both errors were of the same order of magnitude as the rmse achieved with a sigmoid NN in Ref. ${ }^{187}$.

Brown and Pradhan have used EXPNN to fit ab initio data to make PESs for $\mathrm{CS}_{2}$, HFCO and HONO from ab initio data. ${ }^{242-246}$ Fitting $\mathrm{CS}_{2}$ PES to CASPT2 data, ${ }^{247}$ they observed that an EXPNN SOP PES requires fewer parameters than POTFIT to achieve similar global potential accuracy. A test set rmse of $1.6 \mathrm{~cm}^{-1}$ was obtained for points below $20,000 \mathrm{~cm}^{-1}$ with 30 terms (exponential neurons). ${ }^{242}$ An EXPNN PES was constructed for HFCO in Ref. ${ }^{243}$; the PES, which was fitted to 15,000 CCSD(T) data, covered multiple minima and transition states and had an rmse of $130 \mathrm{~cm}^{-1}$ up to $40,000 \mathrm{~cm}^{-1}$. The NN had 100 neurons. A PES for the same molecule was constructed in Ref. ${ }^{244}$ with an rmse of $10 \mathrm{~cm}^{-1}$ up to $10,000 \mathrm{~cm}^{-1}$, for the purpose of computing vibrational spectra of HFCO and DFCO. 75 exponential neurons were used. The HONO PES covering multiple minima and transition states was fitted to $\operatorname{CCSD}(\mathrm{T})$ data to an rmse of $10 \mathrm{~cm}^{-1}$ up to $10,000 \mathrm{~cm}^{-1}$ with 90 exponential neurons in Ref. ${ }^{245}$. For $\mathrm{CS}_{2}$ and HFCO, the EXPNN PESs were tested by doing MCTDH calculations.

Koch and Zhang proposed an alternative way of making a SOP PES from a NN. ${ }^{101}$ Their SOP PES is built differently. Rather than having an activation function whose argument is a sum, they have a product of activation functions each evaluated for a particular coordinate. To achieve the SOP form, they are not required to use an exponential activation function. They fitted the PES of the $\mathrm{S}_{1}$ state of $\mathrm{NOCl}$ to an rmse of $30 \mu \mathrm{Ha}\left(6.6 \mathrm{~cm}^{-1}\right)$ with 30 terms. EXPNN has the advantage that the SOP form, and therefore easily computable integrals involving the PES, is preserved if one uses coordinates, such as symmetry adapted coordinates, which are linear combinations of the original coordinates. It is lost, however, if symmetry adapted coordinates are linear combinations of products of the original coordinates. ${ }^{23,158}$ More hidden layers can be used if they are linear, such as the linear coordinate-transforming layers in the Red-RS-HDMR-NN scheme ${ }^{115}$ (however, only one non-linear layer should be used to preserve the SOP property in the original coordinates). This is not true in general for multiplicative NNs. ${ }^{101}$ This is generally not true also with some other approaches representing the PES with component functions, in particular, those representing the PES as a sum of environment-dependent atomic contributions. ${ }^{50,61,120,130,135}$ 


\subsection{Diabatic PES}

For some photodissociation and reaction processes, it is necessary to consider more than one PES. The PESs for which we have discussed NN fitting methods in other sections are a result of the Born-Oppenheimer approximation. The electronic energies to which a NN is fit are obtained by solving the electronic Schrödinger equation at a set of geometries. The nuclear dynamics is then studied on the resulting PES. This separation into electronic and nuclear problems is accurate when different electronic energies are well separated. When separated products are in electronically degenerate states, there are always points on the ground state BO PES at which non-adiabatic coupling between BO PESs is important. In principle, it would be possible to separately fit, for example with one or more NNs, all relevant BO PESs and the non-adiabatic kinetic coupling terms and to use them to study dynamics. In practice, this is difficult because the BO PESs may not be smooth. The most obvious manifestation of their non-smoothness is conical intersections. ${ }^{74,248,249}$

Rather than fitting adiabatic BO PESs, it is more common to fit what are called diabatic PESs. Associated with a diabatic PES is a diabatic electronic wavefunction. Each member of a set of $K$ perfect diabatic wavefunctions is a linear combination (with coordinate-dependent coefficients) of $K$ of BO electronic wavefunctions and the coefficients are chosen so that kinetic coupling matrix elements are zero. ${ }^{250}$ For polyatomic molecules it is in general impossible to form such diabatic functions. There are nevertheless methods for making nearly diabatic functions for which the kinetic coupling is assumed to be small. ${ }^{249}, 250$ In principle, it would be possible to compute a set of adiabatic electronic wavefunctions and to transform them to obtain diabatic wavefunctions and the associated diabatic PESs. It is more common to bypass the transformation by using a method often called Diabatization by Ansatz. ${ }^{250}$ Several authors have used NNs and Diabatization by Ansatz to fit diabatic PESs. Diabatization by Ansatz ${ }^{250}$ is a trick for extracting diabatic PESs from points on adiabatic PESs, to which one has access with standard quantum chemistry packages. To form a set of $K$ diabatic states, the elements of a $K$ by $K$ diabatic representation are parameterized by one or more NNs. The parameters are adjusted to reduce the difference between the eigenvalues of the diabatic matrix and electronic energies on BO PESs. In this way diabatic PESs are obtained from quantum chemistry calculations without knowing, before the diabatic PESs are determined, the adiabatic-diabatic transformation matrix. Diabatic PESs are 
smooth. There are no avoided crossings. The associated wavefunctions change slowly as the geometry of the nuclei changes. Because the diabatic PESs are smooth, they can be fit with NNs.

Many papers report fits of BO PESs, but there is much less work done on fitting diabatic PESs. For decades, the elements of the diabatic matrix elements were represented with Taylor series and the coefficients were fit using the Diabatization by Ansatz idea. ${ }^{250}$ Each matrix element is a simple sum of monomials. The groups of Manthe and Eisfeld have recently used NNs to fit elements of the diabatic matrix. Lenzen and Manthe used one NN with multiple outputs to fit all of the matrix elements. They have fit PESs for $\mathrm{H}_{2}+\mathrm{Cl} \rightarrow \mathrm{H}+\mathrm{HCl}$ and $\mathrm{Cl}\left({ }^{2} \mathrm{P}\right)+\mathrm{CH}_{4} \rightarrow \mathrm{HCl}+$ $\mathrm{CH}_{3}{ }^{251}{ }^{252}$ In their for $\mathrm{H}_{2}+\mathrm{Cl} \rightarrow \mathrm{H}+\mathrm{HCl}$ paper they use two hidden layers. ${ }^{251}$ If the correct symmetry is not imposed, they observe that their diabatic PESs are missing a conical intersection. When they force off-diagonal matrix elements to have the correct symmetry properties, the conical intersection is obtained. Lenzen and Manthe mention that optimization of the NN is complicated by the fact that the output of the NN is not itself an adiabatic energy. The adiabatic energies are obtained from the outputs by diagonalization. For the $\mathrm{Cl}\left({ }^{2} \mathrm{P}\right)+\mathrm{CH}_{4} \mathrm{PES}$, a NN with symmetrized input is used. Each element of the diabatic matrix is a sum of NNs with a single hidden layer. Cuts through the adiabatic PESs obtained from the diabatic PESs show that they reproduce the ab initio points well. Williams and Eisfeld build diabatic PES for $\mathrm{NO}_{3} .{ }^{253,254}$ Rather than representing the off-diagonal elements of the diabatic matrix with a NN, they model a correction factor with a NN. They begin with a standard diabatic model in which off-diagonal matrix elements are sums of monomials and they add to the coefficients a NN correction term. They used a single hidden layer. They state that deeper NNs were not advantageous.

The Diabatization by Ansatz approach yields a matrix representing the electronic Hamiltonian whose eigenvalues are equal to the diagonal elements of the electronic Hamiltonian in the adiabatic basis. In this approach, it is assumed, because the diabatic PESs are smooth, that kinetic coupling in the diabatic representation is negligible. Instead of using Diabatization by Ansatz, one can choose diabatic functions so that the eigenvalues of the diabatic representation of the electronic Hamiltonian are nearly equal to the adiabatic PESs (computable with quantum chemistry methods) and so that derivative coupling in the diabatic representation is nearly zero. This strategy is pursued by other authors. ${ }^{255,256}$ Yin, Guan, Fu, and Zhang apply these ideas to a two-state problem. ${ }^{257,258}$ For a two-state problem, the transformation that relates the adiabatic and 
diabatic representations is determined by a single mixing angle. The elements of the matrix that represents the electronic Hamiltonian are chosen to minimize kinetic coupling in the diabatic representation and so that the eigenvalues of the matrix are close to the adiabatic PESs. They apply these ideas to $\mathrm{ClH}_{2}$ and LiHF. For LiHF, rmse values for adiabatic ground and excited states PESs, fitted to about 2,500 MRCI points, were 1.7 and $4.7 \mathrm{meV}$, respectively (14 and $30 \mathrm{~cm}^{-1}$, respectively), but the rmse for the energy difference between eigenvalues of the diabatic matrix for the electronic Hamiltonian is close to $0.1 \mathrm{eV}$, indicating that derivative coupling in the diabatic representation is not as small as desired. The quality of the diabatic PESs is tested by computing reaction probabilities. ${ }^{257}$ When non-adiabatic effects are unimportant, the adiabatic reaction probabilities agree well with those they compute on two diabatic PESs. For $\mathrm{HCl}+\mathrm{H} \rightarrow \mathrm{Cl}+\mathrm{H}_{2}$, rmse values of the two states' PESs were on the order of $0.05 \mathrm{eV}\left(400 \mathrm{~cm}^{-1}\right)$; reaction probabilities were also computed and good agreement was found between the calculation on two coupled diabatic PESs and the calculation on a single adiabatic PES at low energies, when non-adiabatic effects are unimportant. ${ }^{258}$ Another LiFH fit was done using a different strategy for ensuring that kinetic coupling in the diabatic representation is small. ${ }^{255}$ In this paper, a NN whose three outputs are three elements of the diabatic matrix are used. The fit criterion depends not only on the difference between eigenvalues of the diabatic matrix and ab initio adiabatic energies but also on differences of gradients and interstate couplings computed from the diabatic model and ab initio. The rmse values for the states were on the order of $3 \mathrm{meV}\left(25 \mathrm{~cm}^{-1}\right)$. The quality of the new diabatic matrix was established by comparing reaction probabilities computed with it with those obtained from a previous LiFH fit. ${ }^{259}$ A final improvement of the fit method used $g^{(\mathrm{I}, J)}$ and $h^{(\mathrm{IIJ})}$ vectors as coordinates in order to describe better the diabatic matrix elements in the vicinity of a conical intersection. ${ }^{256}$

The PIP-NN method can be used to enforce symmetry constraints during the fitting of diabatic PESs, in particular, to ensure that the off-diagonal elements of the diabatic matrix change sign with the permutation of like atoms. ${ }^{260}$ The off-diagonal elements can be represented as a PIPNN function multiplied by an antisymmetric factor. This approach was applied to study absorption spectra and non-adiabatic dissociation of $\mathrm{H}_{2} \mathrm{O}(\tilde{X} / \tilde{B})$ and $\mathrm{NH}_{3}(\tilde{X} / \tilde{A}) .{ }^{260}$ The rmse values for the diagonal and off-diagonal elements of the potential energy matrix were on the order of 3-10 meV (24-80 $\left.\mathrm{cm}^{-1}\right)$ for both molecules. The PIP-NN approach was also used to construct a diabatic 
Hamiltonian for the $1,2{ }^{1} \mathrm{~A}$ states of $\mathrm{NH}_{3}$ coupled by a conical intersection seam accurate to dozens $\mathrm{cm}^{-1} .{ }^{256}$ Dipole moment surfaces for these states were fitted with a NN in Ref. ${ }^{261}$; the rmse values were reported as being several per cent. In Ref. ${ }^{262}$, Guan and Yarkony used neural networks to make an analytical representation of spin-orbit coupling interactions in a diabatic basis: the singlet $S_{0}$ and $S_{1}$ states of formaldehyde are diabatized and then the spin-orbit coupling between singlet and triplet $T_{1}$ states is transformed to the diabatic representation. The resulting smooth diabatized spin-orbit couplings are accurately fitted with NNs, with rmse values for spin-orbit coupling matrix elements on the order of $10^{-2} \mathrm{~cm}^{-1} .{ }^{262}$ The SchNet ${ }^{130}$ approach has recently been used to fit potential energy surfaces (energy and forces) and non-adiabatic couplings in excited state dynamics studies. ${ }^{263}$

\subsection{Representations in orders of coupling and with sub-dimensional component functions}

Neural networks have been used to build terms of many-body expansions. Manzhos et al. fitted the three-body term of the nine-dimensional PES for $\mathrm{CO}-\left(\text { para- }-\mathrm{H}_{2}\right)_{\mathrm{n}}$ and $\left(\mathrm{H}_{2}\right)_{3}$ clusters in the rigid rotor approximation. ${ }^{165}$ The NN approach was used to fit the difference between $\operatorname{CCSD}(\mathrm{T})$ data and an existing empirical two-body potential. This leverages the power of both the empirical method (correct overall shape) and the black-box method (ability to fit fine details of a multivariate function). Refitting the difference also limited effects due to overfitting as their magnitude was contained. The PESs were fitted to 10,000 symmetry unique ab initio points with the Red-RSHDMR-NN method (Eq. 8). ${ }^{115,116}$ The test (fit) mae was $0.24(0.23) \mathrm{cm}^{-1}$ and $0.38(0.36) \mathrm{cm}^{-1}$ for $\left(\mathrm{H}_{2}\right)_{3}$ and $\mathrm{CO}-\left(\mathrm{H}_{2}\right)_{2}$, respectively. A similar approach was used by Xu et al. who used an empirical two-body term for the $\mathrm{He}+\mathrm{H}_{2} \rightarrow \mathrm{HeH}+\mathrm{H}$ reaction, and fitted the three-body term with a NN. ${ }^{264}$ The three-body term was fitted with about 10,200 MRCI points to an rmse of $0.095 \mathrm{kcal} / \mathrm{mol}$ (33 $\mathrm{cm}^{-1}$ ) and used in quasiclassical trajectory studies. The three-body term of the PES for the $\mathrm{N}^{+}\left({ }^{3} \mathrm{P}\right)$ $+\mathrm{H}_{2} \rightarrow \mathrm{NH}^{+}+\mathrm{H}$ reaction was fitted with the PIP-NN method in Ref. ${ }^{265}$ and time-dependent wave packet calculations were performed of the rovibrational state distributions and state resolved angle distributions of the product and of the isotopic effect on them. The PES was fitted to about 30,000 MRCI points to an rmse of $2.7 \mathrm{meV}\left(22 \mathrm{~cm}^{-1}\right)$. Earlier, a NN was used to fit the polarization part of the PES of the water dimer on top of an empirical two-body (rigid molecule) potential. ${ }^{266}$ In Ref. ${ }^{267}$, a 6D PES of $\mathrm{H}_{2}$ on $\mathrm{Pd}(111)$ was constructed by first subtracting three-dimensional potentials of individual $\mathrm{H}$ atoms interacting with the surface (which were fitted with a symmetry- 
adapted spline algorithm) and NN fitting the difference. This procedure reduced PES corrugation and simplified NN fitting. A mae of $70 \mathrm{meV}\left(565 \mathrm{~cm}^{-1}\right)$ vs DFT energies was obtained.

In Ref. ${ }^{114}$, we introduced the RS-HDMR-NN method of Eqs. 6-7 and used it to refit analytic an PES of $\mathrm{HOOH}$. The PES was fitted to 5,000 symmetry unique points and its quality tested by computing the vibrational spectrum. The test rmse and spectrum error showed convergence with the order of HMDR, as shown in Table 1. Specifically, the spectrum converged at the $4^{\text {th }}$ order of coupling, which is consistent with Rauhut's calculation. ${ }^{36}$ We also showed in that work that a given dataset limits the maximum order of coupling that can be reliably recovered in a fit. This is illustrated in Figure $\mathbf{1}$ where a measure of relative uncertainty of NN parameters of the component functions of the $(\mathrm{M}+1)$ th order is shown as well as that of the component functions of up to Mth order, when fitting the PES with different orders ( $d$ in Eqs. 6-7) of HMDR. We believe this is a powerful justification for use of lower-dimensional functions: with finite density of sampling, there may not be enough information in the data to recover a genuine Ddimensional function.

Table 1. Test set rmse of the PES of $\mathrm{HOOH}$ and the mae of the first 48 vibrational levels of $\mathrm{Ag}_{\mathrm{g}}$ symmetry of on top of the ZPE with different orders of RS-HDMR-NN computed in Ref. ${ }^{114}$. The numbers are in $\mathrm{cm}^{-1}$.

\begin{tabular}{cccccc}
\hline Order of HDMR & 2 & 3 & 4 & 5 & 6 \\
\hline PES test rmse & 156 & 23.4 & 8.9 & 7.7 & 7.4 \\
Spectrum mae & 10.35 & 1.14 & 0.44 & 0.37 & 0.35 \\
\hline
\end{tabular}




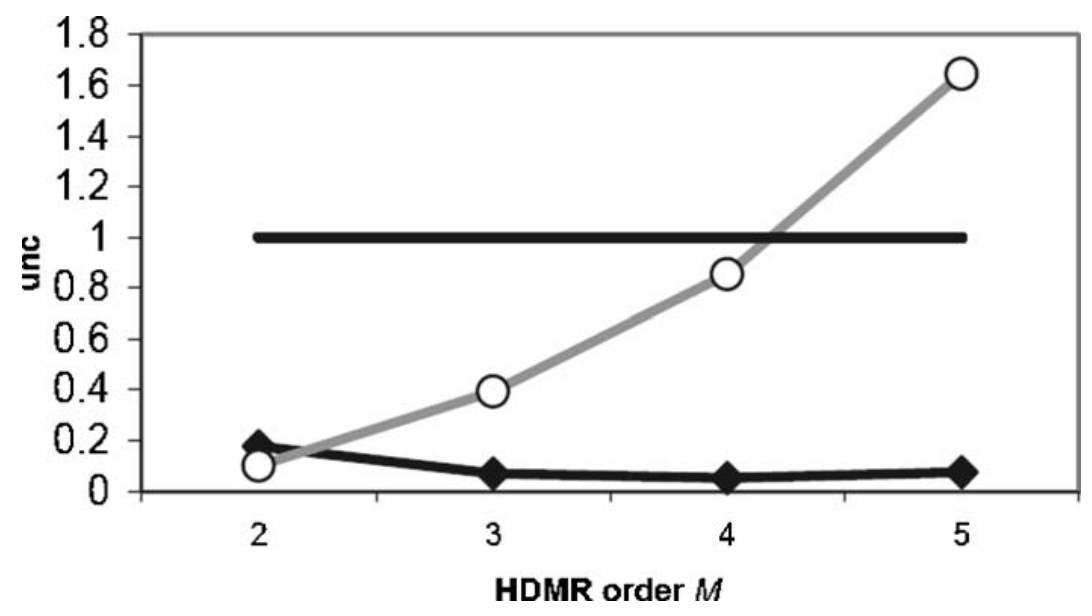

Figure 1. The uncertainties of $\mathrm{HOOH} N \mathrm{~N}$ parameters. Black diamonds: uncertainties of all parameters of an $M^{\text {th }}$ order HDMR. Open circles: uncertainties of parameters of the $(M+1)^{\text {th }}$ mode term. The connecting lines are to guide the eye. The black line at unc $=1$ is a "certainty limit" standard. Adapted from Ref. ${ }^{114}$ with permission from AIP.

In Ref. ${ }^{115}$ we introduced the Red-HDMR-NN method of Eq. 8 and tested it by refitting reference PESs of $\mathrm{HOOH}$ and of the reaction $\mathrm{OH}+\mathrm{H}_{2} \rightarrow \mathrm{H}_{2} \mathrm{O}+\mathrm{H}$ to demonstrate that one can decouple the numbers of terms and their dimensionality. The PES of $\mathrm{HOOH}$ was refitted to 5,000 symmetry unique points. A test set rmse of $12.8 \mathrm{~cm}^{-1}$ was achieved with $212 \mathrm{D}$ terms and a rmse of $10.2 \mathrm{~cm}^{-1}$ with 12 3D terms. The PES of $\mathrm{OH}+\mathrm{H}_{2} \rightarrow \mathrm{H}_{2} \mathrm{O}+\mathrm{H}$ was refitted to about 5,400 points; a test set rmse of $5 \mathrm{~cm}^{-1}$ was achieved with $102 \mathrm{D}$ terms. In Ref. ${ }^{177}$ we showed, refitting the PES of vinyl bromide in 12 and 15 dimensions (in bond coordinates to a reference PES or in interatomic distances to ab initio data, respectively), that with Eq. 8 one can achieve a desired accuracy by either increasing the dimensionality of terms or their number or both. About ten two-dimensional component functions achieve a test set rmse of less than $500 \mathrm{~cm}^{-1}$ in 12D, a far cry from the 66 second-order functions that would be required in standard HDMR and would achieve a worse accuracy. ${ }^{177} 20$ four-dimensional functions achieved a test rmse of $137 \mathrm{~cm}^{-1}$. The trend of the test set error with the number and dimensionality of terms in reproduced in Figure 2. In a standard HDMR or multimode expansion, there would be 495 terms. In 15D and fitting ab initio data, 15 six-dimensional component functions achieved a test set rmse of $769 \mathrm{~cm}^{-1}$, which was at that time limited by fitting only subsets of the data due to limited CPU power. In a standard HDMR or multimode expansion, there would be 5,005 terms. We also showed that as the number of available PES samples increases, fits with higher-dimensional component functions become more reliable. 
These works demonstrated that the combinatorial scaling of the number of terms of the HDMR expansion is effectively avoided with the Red-RS-HDMR-NN method.

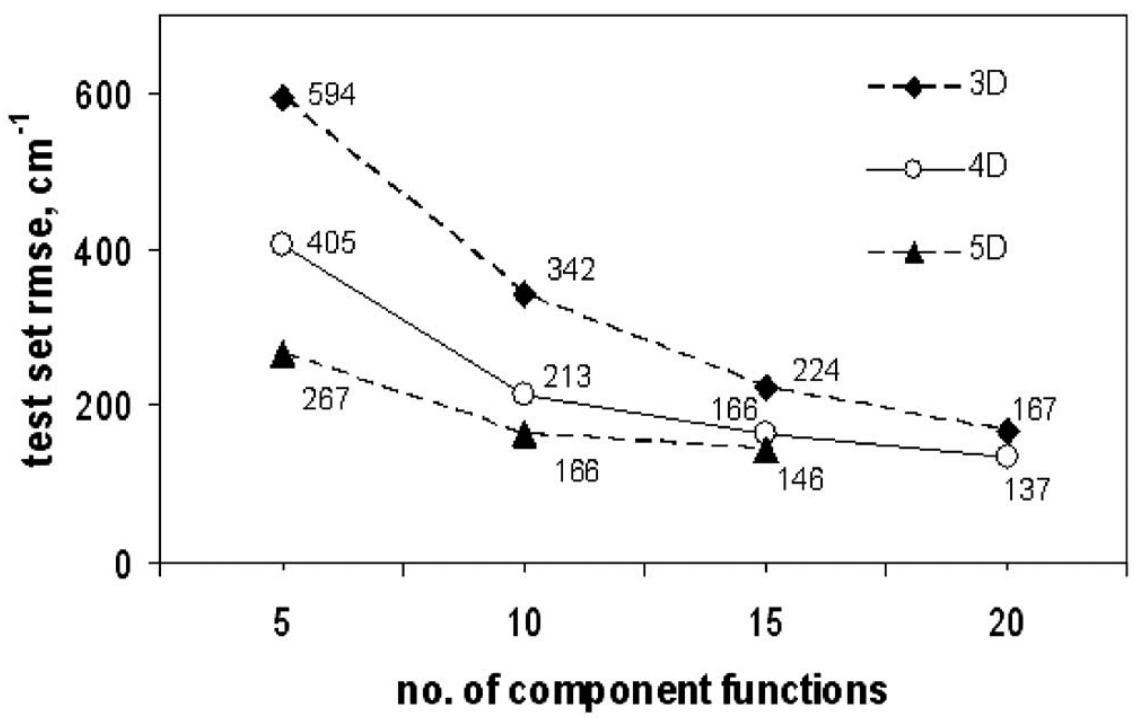

Figure 2. The rmse of the test set as a function of the number of three-, four-, and five-dimensional component functions in Red-RS-HDMR-NN for 12-dimensional fits of the vinyl bromide PES with 20,000 points. The connecting lines are to guide the eye. Reproduced from Ref. ${ }^{177}$ with permission from AIP.

Raff and co-workers used a many-body representation with neural networks. ${ }^{189}$ They represented terms of a many-body expansion with (single hidden layer) neural networks and also addressed the problem of the combinatorial explosion of the number of terms. They used the socalled moiety-energy (ME) approximation to reduce the number of terms. Equivalent sets of atoms are defined which are sets of atoms in which the same number of each type of atom is present with similar bonding characteristics, and all equivalent sets are represented with a single NN. With this approach, they fitted the PESs $\operatorname{Si}_{n}$ of clusters $(n=3,4, \ldots, 7)$ to a large number of DFT data and the dissociative PES of vinyl bromide ${ }^{187}$ in 15 dimensions. They found that an expansion truncated after the three-body terms is sufficient to fit the $\mathrm{Si}_{5} \mathrm{PES}$ with a mae test set error of $5.693 \times 10^{-4} \mathrm{eV}$ (only $4.6 \mathrm{~cm}^{-1}$ ). For other clusters test set errors were up to $0.0212 \mathrm{eV}$ (about $170 \mathrm{~cm}^{-1}$ ). For vinyl bromide, a fourth order many-body expansion provided a mae about $0.08 \mathrm{eV}$ (about $650 \mathrm{~cm}^{-1}$ ). These errors were only marginally higher than those of full-dimensional fits.

In Ref. ${ }^{66}$, Behler type $\mathrm{NN}^{50,118}$ was used to fit two- and three-body terms of the manybody expansion representing water interactions. The potentials were fitted to $\operatorname{CCSD}(\mathrm{T})$ data for 
$\left(\mathrm{H}_{2} \mathrm{O}\right)_{\mathrm{n}}$ clusters with $n=4-6$; about 42,000 points were used to sample two-body terms (defined as dimers) and about 12,000 points to sample three-body terms (defined as trimers). At the twobody level the fit could reproduce the ab initio energies with a test set rmse of $0.079 \mathrm{kcal} / \mathrm{mol}(6.6$ $\left.\mathrm{cm}^{-1}\right)$ and at the tree-body level with a test set rmse of $0.063 \mathrm{kcal} / \mathrm{mol}\left(5.3 \mathrm{~cm}^{-1}\right)$.

Yao et al. also used NNs to fit terms of a many-body expansion. ${ }^{166}$ Each body term was fitted with a multilayer $\mathrm{NN}$ as a function of Coulomb matrix ${ }^{131}$ based descriptors as well as a novel descriptor vector they proposed called “depth map”. They tested their method by fitting MP2 data for interacting methanol molecules. The total data set included 844,800 methanol molecular structures for one-body energies, 74,240 methanol dimer structures for two-body energies, and 36,864 methanol trimer structures for three-body energies. Two- and three-body terms were fitted with a mae of about 15 and $20 \mathrm{mHa}$, respectively (3.3 and $4.4 \mathrm{~cm}^{-1}$, respectively).

\subsection{Dimensionality reduction}

The intrinsic dimensionality of the data sampling the PES may be lower than $D$ not only due to finite density of sampling, but because the original coordinates are redundant. This is the case, in particular, with interatomic distances $R_{i j}$ widely used in PES fitting due to convenience of treating symmetry and asymptotics. ${ }^{23,24,158,172-174,198}$ For a molecule, the dimensionality of the vector of $3 N_{a}-6$ coordinates is the intrinsic dimensionality of the configuration space, where $N_{a}$ is the number of atoms. The dimensionality of the vector of $R_{i j}$ coordinates is $N_{a}\left(N_{a}-1\right) / 2$, and these coordinates are redundant for free molecules with more than four atoms. For molecules with frustrated translation / rotation, such as molecules on surfaces, this redundancy appears even for smaller molecules. The use of (symmetry adapted) functions of coordinates in the PIP-NN method, ${ }^{198}$ in the FI-NN method, ${ }^{159}$ and in the method of Behler ${ }^{50,120}$ also leads to redundant inputs. ${ }^{142,}{ }^{170}$ It is in principle possible to reduce the data to the intrinsic dimensionality and fit the PES in $D_{\text {intr }}$ without loss of accuracy. Even when the coordinates are not redundant, if only a part of space is of interest, the intrinsic dimensionality of data sampling that part, even densely, may be lower than $D$. This is the case, in particular, for reactive potentials where often only the part of the PES around the minimum energy path is of interest.

Dimensionality reduction of potential energy surfaces has been explored before, ${ }^{268}$ including in the context of methods used for PES construction such as RKHS. ${ }^{269}$ Advantages of D-reduction in MD simulations have been demonstrated, in particular, in efficiency of sampling 
the parts of space which are of interest. ${ }^{270}$ D-reduction can be linear or non-linear. Principal component analysis (PCA) is a simple example of linear D-reduction which has been used in MD. ${ }^{270}$ To reduce the dimensionality of complex data sets such as samples along a minimum energy path, in general non-linear D-reduction techniques are called for such as LLE (locally linear embedding) ${ }^{271}$ or MDS (multidimensional scaling). ${ }^{272}$ An NN autoencoder is a non-linear Dreduction techniques. It has been used for molecular PES representation by Herr et al. ${ }^{273}$ They used an autoencoder to produce a smaller set of latent variables describing atomic properties, which were then used as inputs in the NN approach of Behler ${ }^{50,53,57,120}$ to build neural network potentials of a large number of molecules. Dimensionality reduction with Behler type NNs was also used in solid state. ${ }^{274}$

We noted in section 2.2 that a multimode NN naturally gives the possibility to perform dimensionality reduction. The Red-RS-HDMR-NN approach described in section $3.5^{115-117}$ also offers this possibility. Dimensionality reduction is realized when the total number of coordinates in Eq. 8 is smaller than the dimensionality of the configuration space, $N d<D$. For the case of a single component function ( $N=1$ ) it is realized when $d<D$ and the method then bears resemblance to an autoencoder, which is a non-linear D-reduction technique. ${ }^{106}$ The difference with the autoencoder is that in Red-RS-HDMR-NN, the dimensionality reduction is realized with a single linear layer of neurons. ${ }^{115}$ It need not be single or linear, but we have shown ${ }^{115}$ that already with a single linear layer defining the coordinate transformation from $\boldsymbol{x}$ to $\boldsymbol{x}^{(i)}$ in Eq. 8, one can achieve a desired accuracy by increasing either $d$ or $N$, in which case the total set of coordinates (all component of all $\boldsymbol{x}^{(i)}$ ) is redundant. When using Eq. 8 in the dimensionality reduction regime, there is no universal approximator property, and the quality of the fit is then dependent on the intrinsic dimensionality of the training data, which with finite density of sampling is always lower than the space dimensionality $D$.

In Refs. ${ }^{116,163}$, we performed dimensionality reduction of the PES for the reaction of dissociative adsorption of $\mathrm{N}_{2} \mathrm{O}$ on $\mathrm{Cu}$ with a $\mathrm{NN}$ implementing Eq. 8. All molecular degrees of freedom were considered while the copper surface was frozen. The PES was fitted in 15 inverse interatomic distances among the atoms of the molecule and between the three atoms of $\mathrm{N}_{2} \mathrm{O}$ and four atoms at the surface of copper. This is a redundant set, as the intrinsic dimensionality of the space is nine ( $3 N_{a}$ as rotation / translation are frustrated near the surface). In Figure 3, we reproduce the results of fitting Red-RS-HDMR-NN with $N=1$ and different $d$ to data sampled on 
this PES (sampling details are given in section 3.7). The trend with $d$ of the test set error is shown. The test error levels off about $d=8-9$, i.e. around the intrinsic dimensionality. It is encouraging that the intrinsic dimensionality was found by the algorithm using a linear coordinate transformation even though the transformation between non-redundant bond coordinates or Cartesian coordinates (non-redundant in this case) and interatomic distances is non-linear. This suggests that approximate non-redundant coordinates can be found with a linear transformation affected by a layer of linear neurons of a NN, akin to PCA. That there is no significant decrease in error already from $d=8$ is a reflection of the low density of sampling - this PES was fit with a DFT sampling density close to 2 data points per DOF - which further decreases the intrinsic dimensionality of the data and justifies the use of D-reduction which effectively concentrates the data and reduces the danger of NN overfitting.

Dimensionality reduction was also realized when fitting, with the same Red-RS-HDMRNN method, the nine-dimensional PES of CO- $\left(\text { para- } \mathrm{H}_{2}\right)_{\mathrm{n}}$ and $\left(\mathrm{H}_{2}\right)_{3}$ clusters. ${ }^{165}$ The fit was performed in redundant interatomic distances, in a 15-dimensional space. The algorithm correctly identified the intrinsic dimensionality of nine.

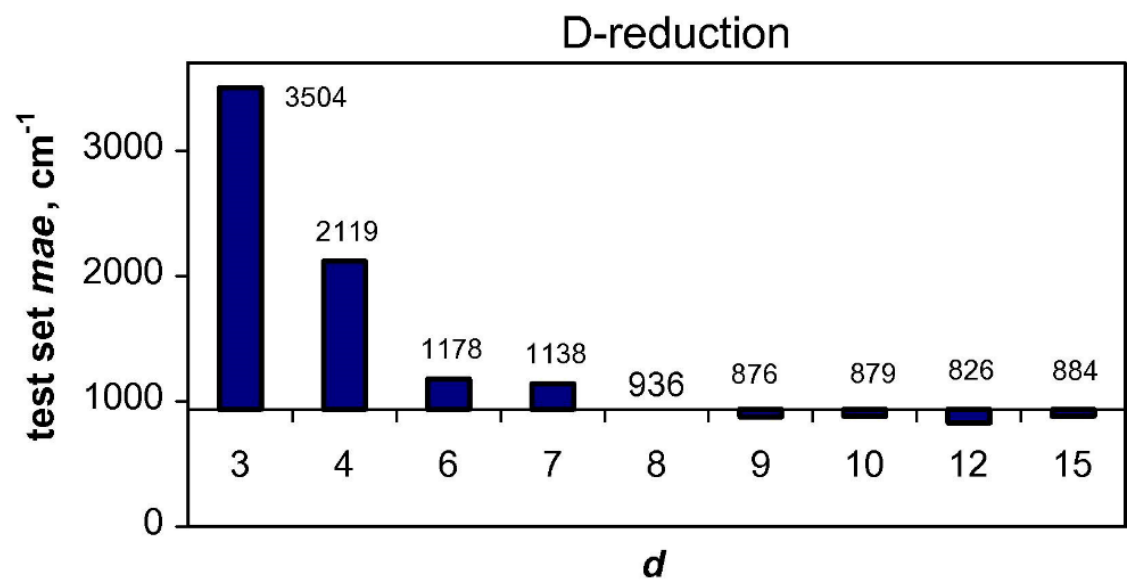

Figure 3. The mean absolute error at the test points as a function of the dimensionality of the neural network when fitting the PES for $\mathrm{N}_{2} \mathrm{O}$ dissociation on $\mathrm{Cu}$. The results are optimized with respect to the number of neurons. The "floor" level corresponds to the best fit at the intrinsic dimensionality $d=8$. Adapted with permission from Elsevier from Ref. ${ }^{163}$.

\subsection{Data selection and meaningfulness of fitting and test errors}

The sampling of configuration space is a critical step in PES construction with NN based methods, as with any method. The distribution of fitting and test points in space determines the quality of 
the fit and the meaningfulness of the fit or test error in applications. In multidimensional spaces, the cost of ab initio calculations is typically higher (as the molecule is bigger) and the density of sampling is much lower than in a lower-dimensional case. Positioning of the points in configuration space is therefore of utmost importance. Many of the sampling approaches used for NN potentials are also useful when building PESs with other methods. We will review several which have been shown to work well and can be recommended for future NN PESs.

When the PES is intended for calculation of spectra, the most important regions of the configuration space are near the minima. These regions contribute to all vibrational levels while higher-energy parts of the potential only influence higher-lying states. It is the lowest energy (however many) vibrational states which are typically accurately measured in experiments, and are of interest to computational chemistry. It is therefore desirable to obtain the lowest potential values in the well around the minimum very accurately; the error tolerance for potential values near the classical turning points of the highest-energy levels of interest can be higher and much higher for values much above the turning points. A natural sampling scheme that satisfies these requirements uses an inverted potential probability density function to select fit and test points. This is done by randomly selecting points in space and are accepting a point into the fitting or test set if its potential value $V$ satisfies

$$
\frac{V_{\max }-V}{V_{\max }-V_{\min }}>\text { rand }
$$

where rand is a (uniformly distributed) random number on [0,1], and $V_{\max }$ and $V_{\text {min }}$ are the desired maximum and minimum values of the potential. This idea comes from the placement of quasirandom distributed Gaussian basis functions suggested by Garashchuk and Light. ${ }^{275}$ It naturally emphasizes more important lower-energy regions of the potential. It acts on the distribution of $V$ values rather than on the distribution of the variables and works wells with any coordinates in any dimensionality. Eq. (8) strictly implements an inverted potential point distribution; it can be modified to suit better the problem at hand as:

$$
\frac{V_{\max }-V+\Delta}{V_{\max }-\left(V_{\min }-\kappa\right)}>\text { rand }
$$


where $\Delta$ ensures a higher probability of selecting points with energies close to $V_{\max }$ and $\kappa$ ensures a unity probability of acceptance within a finite range above $V_{\min }$, which we found useful for applications with low density of states near the minimum, such as happens in Coulomb-like potentials. ${ }^{276}$ For molecular PESs, we found it unnecessary to use $\kappa$ but a value of $\Delta$ of less than a tenth of the energy range was found to improve the results. ${ }^{69}, 98,114,115,162,165,215,277-282$ Eq. 8 was also used to form NN fitting and test datasets by others. ${ }^{244}$

The underlying points to which Eq. 9 is applied are assumed to be uniformly distributed. A grid is one way to create such underlying uniform distribution. Needless to say, the points selected by Eq. 9 will no longer lie on a grid. Another option is uniform random points. However, when the density of sampling is low, as we have showed it always is in four-atom and larger molecules, random points no longer uniformly cover the space, with large volumes of space having no data. A solution to this issue is the use of pseudorandom sequences such as the Sobol sequence ${ }^{283}$ which we found to work very well ${ }^{68,98,139,215,280,282}$ and which has since been used by others. ${ }^{211}$ Another issue in applying Eq. 9 is that an estimate of $V$ needs to be known before the PES is constructed. In practice, this is not a serious problem as a simple approximation to $V$ is generally sufficient for generating the point distribution. Such an approximation can be obtained by simple fits of a small set of points (which could e.g. be evenly distributed) with a NN or any other method. In the case of a single potential well, a simple harmonic approximation to $V$ will be sufficient in most cases.

The approach of Eq. 9 to emphasize more important low-energy regions does not necessarily work well for reactive potentials, where the PES around the minimum energy path (MEP) needs to be well sampled and different parts of the MEP may differ significantly in energy. Another popular and effective way to select data for NN training, and which satisfies this requirement, is classical molecular dynamics. The advantage of this approach is that it can work well even for multiwell potentials or for reactive potentials. MD can initially be performed on an approximate (e.g. analytic) $\mathrm{PES}^{152,166,197}$ or as direct ab initio MD (AIMD) with an inexpensive approach like DFT $^{121-125}$ or DFTB. ${ }^{284,285}$ This approach is used with non-NN methods, for example, it was bundled with the MSI ${ }^{17-19}$ method and used with IMLS. ${ }^{21}$ A key advantage of using MD-guided point selection is that dynamically important regions of the configuration space are naturally sampled. MD trajectories can also be used to grow the point set dynamically as trajectories "visit" under-sampled points of space. ${ }^{156,170}$ PES constructed with MD-guided point selection are 
naturally advantageous for use in MD simulations. This sampling approach is not designed to be good for quantum dynamics as wavefunctions have significant amplitude in regions that are classically inaccessible. Raff group's pioneered the use of MD-guided sampling to construct reactive NN PESs for vinyl bromide, ${ }^{197} \mathrm{Si}_{4}$ cluster, ${ }^{152} \mathrm{SiO}_{2},{ }^{186} \mathrm{HOOH},{ }^{140}$ and HONO. ${ }^{138}$ They also used AIMD to search for additional configurations to add to the training set. ${ }^{152}$ The Behler group introduced an enhanced version of MD-guided approach: ${ }^{57}$ a given training data set is used for several different NN fits which are expected to be of similar quality. Different NN architectures can be used to guarantee that the functional forms of these fits are different, but their quality is similar. Then, a large number of trial configurations are generated, e.g. by MD simulations based on one of these fits, and the energies of all these configurations are recalculated using all the NNs. The fits are likely to predict very similar energies for those structures, which are close to a training point. If, on the other hand, a structure differs significantly from all training points, the different NNs are likely to predict significantly different energies and forces. This is used to identify structures in poorly represented regions where new data should be added. This procedure can be iterated in an automated way until a desired global PES accuracy is achieved. ${ }^{205}$ A similar approach was used in Ref. ${ }^{286}$. Gradients (forces) can also be explicitly used to guide sampling. Le et al. ${ }^{140}$ introduced a technique that preferentially places new points in regions where the current density of points is insufficient to accurately fit the surface gradients.

The above sampling approaches are usually supplemented with empirically guided sampling, such as placing additional points near the minima or other key parts of the PES to make sure they are not missed from the dataset when using automated methods (for example, minima or transition states may be missed by MD trajectories). ${ }^{17,}{ }^{18}$ We found it was useful to add slices of the PES along coordinate axes. ${ }^{139,162}$ While automated sampling schemes are attractive for their conceptual purity, point selection based on intuition can provide good results. For example, Lorenz et al. showed that by a judicious, intuition guided point selection, it is possible to produce a good NN PES for molecules on surfaces with few samples - on the order of $10^{3}$ for six-dimensional PESs of $\mathrm{H}_{2}$ over clean, sulfur -and potassium-covered Pd. ${ }^{155,169}$

In Ref. ${ }^{116}$, the PES energy surface of $\mathrm{N}_{2} \mathrm{O}$ on $\mathrm{Cu}$ was sampled by random walks in the configuration space emanating from a minimum energy path which was computed by DFT for the dissociative adsorption of $\mathrm{N}_{2} \mathrm{O}$, and fitted with a NN-based method. This approach also allows to emphasize dynamically important regions of space for reactive potentials. 
All the above approaches amount to the introduction, based on physical intuition, of a bias as to which regions of the configuration space will be preferentially sampled. A more radical approach, for the same PES of $\mathrm{N}_{2} \mathrm{O}$ on $\mathrm{Cu}$, was used in Ref. ${ }^{163}$. All Cartesian coordinates of all atoms of the molecule within the simulation cell were uniformly and randomly sampled. The only restriction that was introduced was related to the fact that the breaking of the NN bond was known to not occur in the reaction of interest; therefore, the NN bond length was sampled using an “inverted potential” probability density function as described above, using a simple Morse model. This only served to prevent sampling unnecessarily high-energy configurations without introducing any bias as to the possible dynamics within the energy range considered. DFT calculations were performed at these sampled coordinates and used to construct a PES using the Red-RS-HDRM-NN method as described in section 3.6.

When one performs MD-guided sampling to build a PES meant to be used with MD, the distribution of the training and test points is likely to be close to that of the points on which the PES is called in applications. This is not necessarily the case when an MD-guided PES is used in quantum dynamics. MD-based sampling ignores classically forbidden regions, even though MDbased sampling has been used to generate PESs for quantum dynamics. Even non-MD based sampling methods, including those described above, do not always a produce a point distribution similar to the distribution of the important quadrature or collocation points used in quantum dynamics calculations. Important quantum dynamics points are those at which the wavefunctions one wishes to compute have significant amplitude. It is the error at the important quantum dynamics points that matters. Even if the test points are in the same region as the important quantum dynamics points and even if the distributions are the similar, it is always true that the number of test points is much smaller. This necessarily means that the test points do not completely characterize the PES. Local features of the PES may not influence the test rmse. The small number of fitting data points is compounded by an even smaller number of test points. Although it is understood that using a test set is necessary to control overfitting, it is common in the NN literature to reserve $5-20 \%$ of the data for testing (some examples are given in the next section), and sometimes as few as $2.5 \%$ of the data. ${ }^{120}$ In our experience, this is often grossly insufficient. We found that it is desirable to use at least as many test points as fitting points, and even that does not guarantee the global quality of the PES as the sampling is simply too sparse. 
When using validation (early stopping), although the same set can also be used for testing and validation, the available data are often split into three separate sets for fitting, validation, and testing, which further reduces the number and density of testing (and validation) points. In our experience, we found early stopping not useful: we found that in many cases the validation test error is not monotonic with the numbers of epochs, and that therefore early stopping can prevent achieving the best fit. This ultimately has to do with the low density of sampling of the validation set. In our experience, the best strategy was not to use early stopping but to use a test set as large as possible.

A related issue to data selection is the choice of the size of the NN. To avoid the danger of overfitting, the number of NN parameters must be smaller than the number of data. For example, for a single-hidden later NN fitting only energy (i.e. a single output) in $D$ dimensions, the number of NN parameters is $N_{n}(D+3)+1$, where $N_{n}$ is the number of neurons. The number of parameters can escalate rapidly with $D$ and $N_{n}$ : when fitting four- and five-atom molecules, $N_{n}$ on the order of 150 is often used. $^{68,100,114,115,139,162,177}$ For a five-atom system fitted as a function of interatomic distances $(D=10)$, one works with about 2,000 parameters in a single-hidden later case. If the same number of neurons were used in a two-hidden layer NN (150 neurons in each layer), the number of parameters would balloon to about 24,000. Besides the cost of high-quality (using the Levenberg-Marquardt algorithm) non-linear fitting with this many parameters (which can easily take several hours even on modern computers), one also needs to make sure the number of parameters remains much lower than the number of training points, to make sure that the rmse error is meaningful. Based on the universal approximator property of a single-hidden layer neural network, there is no need to use multiple layers unless they are required to impose a particular physically motivated architecture ${ }^{106,114,115,117,163,165,210}$ or to deal with extremely uneven data distributions. ${ }^{96}$ The consideration of the number of parameters also favors single-hidden layer NNs.

The number of parameters is understandably large in methods using sums of atomic contributions with Coulomb matrix inspired inputs, ${ }^{50,61,130,135,136}$ where the use of feature vectors particular to each atom implies redundant coordinates. Fits with a quarter of a million parameters were reported. ${ }^{136}$ Gastegger et al. addressed the issue of a large number of atom-centered symmetry functions used as inputs in Behler-type NNs by introducing new descriptors called weighted atomcentered symmetry function (wACSF) and optimizing them with a genetic algorithm. ${ }^{287}$ 


\subsection{Controlled comparisons of NNs to other approaches}

As briefly described in section 1.1, there is a plethora of black-box fitting or interpolation methods for PES construction and ample literature reporting applications. There is also by now ample literature on NN potentials, yet there are very few studies comparing NN PESs to PESs built by other methods for the same molecules and on the same data. Such studies are important as only they allow exposing relative advantages and disadvantages of NN based potentials and rational choice of the NN method among the available methods. Ideally, such comparative studies should compare not only fitting errors but also quality of observables computed on the PES, especially, as we have highlighted in the previous section, differences in distributions between fitting and test data on one hand and the points at which the PES is called in applications on the other can make the PES error itself not very informative. Although for many small molecules PESs have been constructed with different methods for the same molecule (including different $\mathrm{NN}$ based methods ${ }^{64}$, 65,162 ), that literature only gives ballpark estimates rather than controlled comparisons which can only be made when fitting the same data. The following examples show why controlled comparisons on the same data are important to avoid a skewed perception of the accuracy of different methods.

In Ref. ${ }^{140}$, a comparative evaluation of $\mathrm{NN}$ and support vector machine (SVM) was reported for fitting of the HOOH PES. They compared the number of parameters and testing set fitting errors of the two methods. Using 34 neurons in the hidden layer, the total number of parameters in the NN fit was 273, and the mae testing set error was $0.0079 \mathrm{eV}$ (about $64 \mathrm{~cm}^{-1}$ ) for the NN. The SVM fit required 3,130 parameters for 447 radial basis function support vectors to produce an mae testing set error of $0.0311 \mathrm{eV}$ (about $250 \mathrm{~cm}^{-1}$ ), i.e. about four times higher. They also used a committee of five NNs; the NN-committee error was five times better than that of SVM. In addition, they showed that integrating a trajectory on a single NN PES is much faster than on a single SVM PES.

In Ref. ${ }^{68}$, the PES of methane was fitted with different methods to the same dataset of about 100,000 high quality ab initio data (computed at the (AE)-MRCI-F12(Q)/CVQZ-F12 level) spanning the energy range beyond $30,000 \mathrm{~cm}^{-1}$ above the minimum. A plain NN approach was compared to a method combining interpolating moving least squares (IMLS) ${ }^{288-292}$ fitting and permutation invariant polynomials (PIP) ${ }^{23,158}$, a method combining PIP and NN (PIP-dependent NN), and a combination of PIP and weighted least-squares (WLS). On all these PESs, highly 
accurate vibrational spectra were computed with full consideration of coupling and an exact kinetic energy operator, using a contracted-iterative method and a Lanczos eigensolver. ${ }^{293-296}$ The potentials were fitted in 10 internucleai distances. In the case of $\mathrm{NN}$, we used a committee ${ }^{98}$ of NNs to reduce the random component of the error.

Although symmetry was taken care of by using PIPs as inputs or basis functions in the other methods, with the plain NN, we used a combination of fitting in a symmetry unique region and averaging near the boundaries. We chose a symmetry unique region in which the $\mathrm{CH}$ distances satisfy $R_{\mathrm{CH} 1}<R_{\mathrm{CH} 2}<R_{\mathrm{CH} 3}<R_{\mathrm{CH} 4}$, and the six $\mathrm{HH}$ distances are in the order $\mathrm{H} 1 \mathrm{H} 2, \mathrm{H} 1 \mathrm{H} 3, \mathrm{H} 1 \mathrm{H} 4$, $\mathrm{H} 2 \mathrm{H} 3, \mathrm{H} 2 \mathrm{H} 4, \mathrm{H} 3 \mathrm{H} 4$. To obtain a PES point in another region we call the NN at the appropriate point in the symmetry unique region. In this fashion, one obtains a NN PES that has the same value at all permutationally equivalent points. However, at the edges of the symmetry unique region, the NN fit may be poorer because only points on one side of the edge are used. Specifically, the derivative of the PES obtained by extending the fit outside the symmetry unique region can be discontinuous across the edge. In the case of $\mathrm{CH}_{4}$, the equilibrium geometry is at the edge of the symmetry unique region. This problem was solved by adding fitting points that are permuted points outside the edges. When the permutation symmetry is high, the data set would become very large if all permuted points were added. Instead, we added all $N_{p}=24$ permutationally equivalent points, but only for ab initio points with energies less than $500 \mathrm{~cm}^{-1}$ above the minimum. When the PES is called, we average values obtained at the permutationally equivalent points.

The equilibrium CH bond length was accurate to $1 \times 10^{-5} \AA$ and harmonic frequencies were similar to within a couple $\mathrm{cm}^{-1}$ with all methods. Specifically, the quality of the NN PESs harmonic frequencies, which are extremely sensitive as the minimum is a high-symmetry point lying on the edge of the symmetry unique region, was as good as those computed on the PIP based PESs. Interestingly, although the different methods resulted in different fitting and test errors, the quality of the vibrational spectra was essentially the same. The root mean square errors (rmse) of the fits with these methods were $1.0 \mathrm{~cm}^{-1}$ with PIP-IMLS, $2.1 \mathrm{~cm}^{-1}$ with PIP-NN, and $3.8 \mathrm{~cm}^{-1}$ with NN, respectively, for energies up to $25,000 \mathrm{~cm}-1$. The calculations of the spectra on the PES using PIPIMLS had an rmse of $4.24 \mathrm{~cm}^{-1}$ vs. the experimental spectrum. The PIP-NN had an rmse of 4.29 $\mathrm{cm}^{-1}$ and that of a committee of simple NNs $3.55 \mathrm{~cm}^{-1}$. All three methods therefore resulted in a similar quality of the vibrational spectra (with the NN PES being in a slightly better agreement with the experiment) which were also within $1 \mathrm{~cm}^{-1}$ of each other. The test sets in that work were 
as small as 5\% of the total number of data points: 5\% in PIP-NN fit, about 25\% in PIP-IMLS and $20 \%$ in NN fits.

It would be wrong to expect errors in observables computed with quantum dynamics on a PES to be similar to the test set rmse reported for that PES. There are two reasons. First, the only points that matter for the quantum calculation are those in the subset of the quadrature or collocation grid points at which wavefunctions have significant amplitude. This subset never coincides with the test points used to assess the error of the PES and therefore the test set error is not a good measure of the error that affects the computation of the observables. This is most important when the important quantum dynamics points are in a region larger than the test point region. Of course, as shown in Ref. ${ }^{220}$, the rmse at a set of points that includes points outside the fitting region may be large. In Ref. ${ }^{220}$ it is 3 about $\mathrm{mHa}\left(660 \mathrm{~cm}^{-1}\right)$ although the error at the training points is much smaller. Second, small errors in the PES (even at the important dynamics points) will have an even smaller effect on observables. This is demonstrated by the methane results. Although the test set errors of the three PESs are somewhat different, the energy levels computed on the three PESs are very close. If the difference between two PESs is $\Delta$, then differences between energy levels computed on them can be obtained from perturbation theory. To first order, levels differ by $\langle\psi|\widehat{\Delta}| \psi\rangle$. For example, if $\Delta$ is oscillatory $\langle\psi|\widehat{\Delta}| \psi\rangle$ may be very small. This means that the error in the spectrum is expected to be smaller than the error in the potential, as long as there are no qualitative errors in the shape (such as discontinuities at the PES minimum described above). In a previous paper, ${ }^{215}$ we found that the rmse of the energy levels was about a factor of 50 smaller than the rmse of the PES when the test is the same as the collocation set. There are no other studies in the literature comparing the rmse at the important quantum dynamics points and the energy level errors and little is known about how the comparison depends on the PES fitting method.

In Ref. ${ }^{215}$, for the first time, we evaluated the accuracy of PESs fit with two methods, at test points that are exactly the PES points used to compute the observables. This was possible due to the use of the rectangular collocation method of Manzhos and Carrington69, 215, 276, 278, 281, 282, 297299 which allows one to compute a spectrum from a relatively small number of potential points which could be the same as the test set used in PES fitting. We refitted a reference PES of $\mathrm{H}_{2} \mathrm{CO}^{300}$ and computed vibrational spectra on the resulting potentials. Reference variational calculations were also performed. We compared the performance of NN and Gaussian process regression ${ }^{40,41}$ (both single NN and GPR and committees thereof) when fitting exactly the same data, in terms of 
potential error as well as in terms of the error in the vibrational spectrum. The results, reproduced in Table 2, were instructive: the average error in the spectrum was up to two orders of magnitude smaller than the average error in the potential at all points used to solve the Schrödinger equation. In the absence of such a comparison, which may be impractical especially with quadrature based methods where grid sizes of hundreds of millions of points are not uncommon, the quality of property calculations is the ultimate arbiter. Simply put, the PES rmse values published in the literature, even in comparative literature (including those in Ref. ${ }^{68}$ ) may not be a reliable measure of the effect of potential fitting error on the spectrum.

Table 2. The rmse (in $\mathrm{cm}^{-1}$ ) of the PES on the test set of 120,000 points and the rmse (in $\mathrm{cm}^{-1}$ ) of the first 50 and 100 vibrational levels of $\mathrm{H}_{2} \mathrm{CO}$ with respect to reference levels computed in Ref. ${ }^{215}$. The number of fitting points is 625 for both the NN and GPR surfaces. For all levels except the ZPE, errors of differences from ZPEs are presented. <..> stands for a committee of 10 NNs or 10 GPRs.

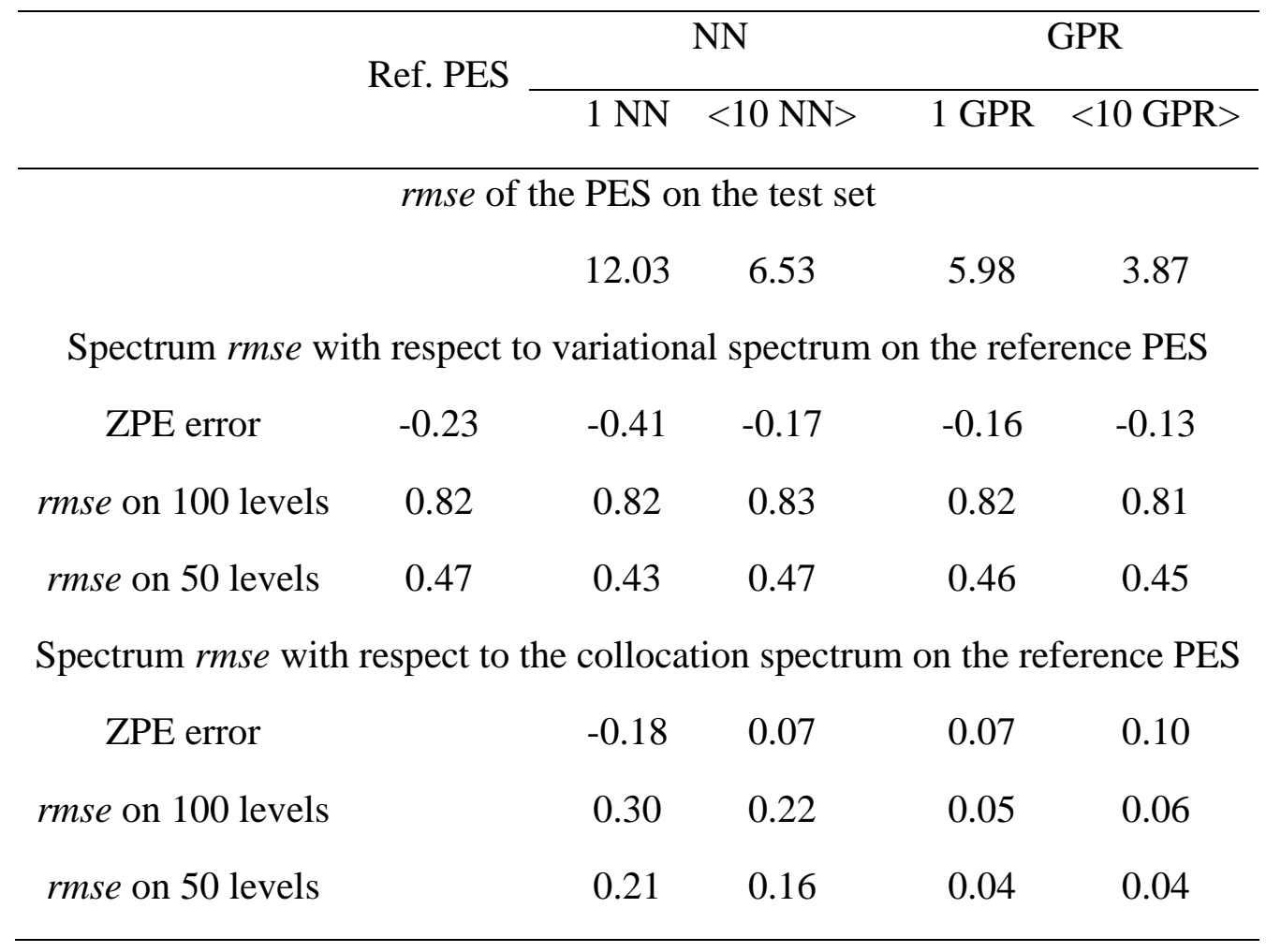

In Ref. ${ }^{66}$, where many-body expansions were used to model water interactions, different methods were used to fit the two- and three-body terms (defined as being due to water dimers and trimers, respectively): the Behler type $\mathrm{NN},{ }^{50,118}$ permutationally invariant polynomials, ${ }^{23}$ and Gaussian approximation potentials. ${ }^{301}$ All three methods showed similar levels of accuracy in 
reproducing both two-body and three-body reference $\operatorname{CCSD}(\mathrm{T})$ data as well as interaction energies of small water clusters, with test set rmse values on the order of $0.05 \mathrm{kcal} / \mathrm{mol}$ (about $4 \mathrm{~cm}^{-1}$ ). In Ref. ${ }^{302}$, the Behler NN method was compared to the systematic molecular fragmentation (SMF) approach ${ }^{303,304}$ which generates overlapping fragments of a certain size by gathering bonded atoms into functional groups. The energy of the total system is calculated by summing the energies of the fragments and subtracting the contributions of the overlap regions. With a benchmark set of all-trans alkanes containing up to eleven carbon atoms, it was found that the NN approach, with a test rmse of $0.00126 \mathrm{kcal} / \mathrm{mol}\left(0.44 \mathrm{~cm}^{-1}\right)$ per atom, yielded smaller errors vs CCSD(T) energies. In Ref. ${ }^{305}$, the Behler NN ${ }^{50,118}$ approach was compared with PIP-NN, ${ }^{23}$ FI-NN, ${ }^{159}$ and a version of PIP-NN using non-redundant PIP (NRPIP-NN) ${ }^{219}$ when fitting PESs for reactions OH + CO, H $+\mathrm{H}_{2} \mathrm{~S}, \mathrm{H}+\mathrm{NH}_{3}, \mathrm{H}+\mathrm{CH}_{4}$, and $\mathrm{OH}+\mathrm{CH}_{4}$ to $74,000,34,000,100,000,63,000$, and 135,000 $\operatorname{CCSD}(\mathrm{T})$ points, respectively. All methods exhibit a similar level of accuracy, which is summarized in Table 3. The large number of PIP inputs for larger systems can also be appreciated from the table. It was found that the Behler method allowed study of systems with larger numbers of atoms, making it more generally applicable than PIP based approaches, which were, however, found to be computationally more efficient for smaller systems.

Table 3. Number of features / rmse values in $\mathrm{meV}\left(\mathrm{cm}^{-1}\right)$ obtained with different NN based fitting methods for the PESs of different reactions in Ref. ${ }^{305}$.

\begin{tabular}{ccccc}
\hline System $\backslash$ method & Behler & PIP-NN & NRPIP-NN & FI-NN \\
\hline $\mathrm{OH}+\mathrm{CO}$ & $48 / 5.8(47)$ & $17 / 5.5(44)$ & $7 / 6.8(55)$ & $7 / 6.7(54)$ \\
$\mathrm{H}+\mathrm{H}_{2} \mathrm{~S}$ & $28 / 5.0(40)$ & $22 / 3.0(24)$ & $9 / 6.9(56)$ & $9 / 5.6(45)$ \\
$\mathrm{H}+\mathrm{NH}_{3}$ & $28 / 3.8(31)$ & $81 / 3.4(27)$ & $38 / 3.6(29)$ & $31 / 4.7(38)$ \\
$\mathrm{H}+\mathrm{CH}_{4}$ & $28 / 8.0(65)$ & $848 / 5.1(41)$ & $203 / 6.3(51)$ & \\
$\mathrm{OH}+\mathrm{CH}_{4}$ & $50 / 4.7(38)$ & $1331 / 3.8(31)$ & & \\
\hline
\end{tabular}

\subsection{Notes on software implementation}

Neural networks have become a method of first resort for high-accuracy multivariate PES construction. Many proprietary or freeware or open source software packages provide NN capabilities, including Matlab, ${ }^{306}$ Octave, ${ }^{307} \mathrm{R},{ }^{308}$ and Python. ${ }^{309,} 310$ There is little need to code the 
basic feedforward NN building block in-house. Available packages can be used as building blocks for a more involved method; that was the road taken by the authors. ${ }^{114-116}$ Recently rather involved NN based packages dedicated to computational chemistry have also been proposed. ${ }^{130,135,311-313}$ Efforts to integrate NN potentials into standard simulation codes have also been made. ${ }^{313}$ We found that the quality of NN fitting can be quite different between codes, and that one should be careful not to make conclusions based on the use of an inefficient algorithm. Specifically, care should be taken when choosing the optimization algorithm. We found that some much-touted in the ML community optimizers such as classical backpropagation with gradient descent ${ }^{314}$ or the more recently introduced Adam optimizer ${ }^{315}$ or other first-order gradient based optimization schemes are simply not good enough to obtain highly (spectroscopically) accurate PES fits. Even if they converge to the desired accuracy, they may require astronomic and unnecessary numbers of epochs; NN fits with tens of thousands of epochs with the Adam optimizer have been reported. ${ }^{316}$ $\mathrm{We}^{68,100,114-116,139,162,163,165,177,215}$ and others ${ }^{140,142,144,145,157,158,167,168,186,194,202,220,257,262,265,317}$ found that it is necessary to use the Levenberg-Marquardt (LM) algorithm. ${ }^{193}$ While the cost per epoch is higher with Hessian-based algorithms, the convergence is much better and the overall cost lower. Our NN PESs reported above were obtained with 500-2,000 epochs, with the error being in the ballpark of the converged error typically within 500 iterations. We recommend reconsidering the algorithm, and if necessary the software, if convergence requires more than 1,000 epochs. Recognizing the high accuracy but also the high CPU cost of the LevenbergMarquardt algorithm, attempts at hybrid solvers have been made. With a combination of the LM algorithm and extreme learning machine (ELM) ${ }^{318}$ it was possible in Ref. ${ }^{319}$ to achieve a rmse for the PESs for $\mathrm{H}+\mathrm{H}_{2}$ and $\mathrm{CH}_{4}$ on $\mathrm{Ni}(111)$ similar to the $\mathrm{LM}$ rmse, but with a fraction of the CPU time. With Behler type NNs, small errors (on the order of $0.1 \mathrm{kcal} / \mathrm{mol}$ or $35 \mathrm{~cm}^{-1}$ for relatively large reactive systems ${ }^{320}$ ) have been achieved with Kalmann filter ${ }^{302,320}$ based optimization, in which every point is presented to the algorithm sequentially, which is advantageous for large systems and datasets. ${ }^{209}$

The availability (or lack thereof) of the LM algorithm is one reason why performance may much differ between software implementations. Our NN PESs reviewed above were built with Matlab. When working on some of them, we tried more than one code, specifically, Octave and Python libraries. In our experience, Matlab’s Neural Network Toolbox (currently called Deep Learning Toolbox) always outperformed any alternative. For other ML methods we found that 
other software and libraries than Matlab were as good or better; this was the case, for example, with Gaussian process regression and genetic algorithms. ${ }^{215,276,321}$ This is an issue to pay attention to, as when comparing the performance of different PES fitting methods, comparisons may be skewed by the efficiency of a particular software implementation; the best software for each method should be used to do justice to the underlying method. ${ }^{215}$

\section{Conclusion}

Having roots in the Kolmogorov theorem dating back to 1957, neural networks have had a long history of development and are now a widely used tool in many areas of science and technology. Like with many other ideas that have revolutionized our lives, such as lithium ion batteries and lasers, decades passed before other developments led to conditions that allowed the idea to shine. In the case of NNs, it was an increase in easily accessible computer power that permitted researchers to make full use of the power of NNs, which, at the conceptual level, had been established in prior decades. Like a basis expansion, a NN is a universal approximator. In fact, one way to think of a NN is as an expansion in a very flexible basis. Because an NN is a universal approximator, it is possible to use NNs to make spectroscopically accurate PESs. However, as recently as the 1990s, NNs were considered a curiosity and dismissed by some as a mediocreaccuracy toy. Today, NN based spectroscopically accurate PESs are routinely made for small molecules, and potentials made with NN based methods are, provided enough points are available, as or more accurate than those made with any other method. This is made possible in particular by the ease with which accurate non-linear optimization with thousands of parameters using millions of data points can be done on modern computers. A critical advantage of NNs and other black-box PES fitting methods is that the computer does the work: there is no need to invest time devising a good fitting function.

NNs have been used to construct PESs for many small molecules and reactive systems and used with classical or quantum dynamics calculations to compute spectra, rate constants, transition probabilities etc. Many of these PESs were reviewed in this paper. It has not been possible in all cases to achieve an accuracy on the order of $\mathrm{cm}^{-1}$, even when the number of atoms is small. This accuracy can now be routinely obtained with plain NNs for four- and five- atom isolated molecules in the region around the potential well(s) (relevant in particular for calculations of vibrational spectra). For reactive systems, and in particular for molecule-surface systems, it is common to 
have rmse values of many hundreds $\mathrm{cm}^{-1}$. NNs being universal approximators, why is it not possible to simple increase the NN size to bring the error down? If an infinitely large number of ab initio points were available, this would indeed be possible. Sampling of the PES is, however, in many cases costly, either because high-level methods are needed for small molecules or because even DFT calculations are costly for molecules on surfaces. What we know for sure is that if the number of fitting points is large enough, a NN is guaranteed to give a good fit. As the cost of generating data decreases, black-box methods that require no tinkering on the part of the user become increasingly attractive.

In actuality, the sampling density is always finite, and in many cases extremely low and this must be taken into account when developing or choosing a PES fitting method. It is ultimately for this reason that more complex (than a plain) NN approaches have been developed such as those reviewed above, including representations with lower-dimensional functions or many-body terms (which may or may not have fewer inputs than the original dimensionality of the configuration space). Related to this is the issue of the distribution of points in configuration space which should favor accuracy not just of the PES at test points but also of observables computed from the PES. The rmse at a set of test (or fitting) points may not be representative of the error at the set of points required to compute the observables of interest. More important is the fact that the error in observables, for example transition frequencies, may be much less than the rmse at the set of points required to compute the observables. ${ }^{215}$ Adding more data points, using more neurons, adjusting the structure of the NN etc. and reducing the rmse of the fitting and test point sets may not improve observables computed on a PES. ${ }^{68,220}$ We found in Ref. ${ }^{68}$ that, even if the test set rmse is slightly larger, the spectrum computed on a plain NN can be as good as the one computed on PESs obtained with more complicated (in terms of both CPU and manpower cost) methods.

Nevertheless, going beyond a simple NN and towards more complex schemes using NNs as building blocks is useful and necessary for dealing with larger systems and systems where data are costly and therefore sparse. With simple NNs, an accuracy of about $1 \mathrm{~cm}^{-1}$ was first obtained for isolated small molecules; ${ }^{162}$ now using methods that take NNs as building blocks, sub-kcal/mol accuracy is routinely obtainable for molecules with dozens of atoms. ${ }^{130}$ 


\subsection{Pros and cons of neural networks}

Neural networks and NN based methods are now widely used to construct potential energy surfaces. The plain NN method is enticingly simple. Due to the existence of pre-packaged codes, it can be used even by researchers or students who know very little about machine learning. More involved schemes like DTNN and others are becoming easier to use as codes are made available to the public. Neural networks are, however, but one of the methods available for fitting a PES with spectroscopic accuracy. Users have an embarrassment of choices including PIP basis, IMLS, GPR, and other methods listed in the Introduction. It is important therefore to point out some advantages and disadvantages of neural networks and derived methods which should help users to make a wise choice.

When choosing a fitting method, the first decision is whether one wishes to use a physically motivated fitting function or a black-box method. For all but very simple PESs, finding a good physically motivated fitting function requires knowing a lot about the system. Many users will instead opt for a general black-box type algorithm. Black-box methods will almost always require significantly more fitting points, but if one can afford to compute the points, they are easy to use. Within the set of black-box methods, NNs have advantages and disadvantages. One disadvantage of NNs is that they extrapolate poorly and therefore do not have correct asymptotic behavior. This disadvantage is, however, shared by all black-box methods and can be palliated by coordinate choices, as described above. NNs can and have been used to fit parameters of a physically motivated functional form which can be chosen to have correct asymptotic behavior. A related disadvantage is lower fit quality at the edge of the sampled space region. This issue can be ignored if borders of the sampled region are in high-energy regions of the potential, but it cannot be if they are not. This is an issue, for example, when fitting points are in a symmetry unique region and its edge is in a low-lying region of the PES. The problem can be solved by sampling (and averaging, as necessary) across the border of the region, as described above. That fact that plain NNs do not

automatically satisfy symmetry constraints is not a disadvantage specific to NNs, it is, like extrapolation, shared by all black-box methods. In this review, we have presented established methods for forcing symmetry onto NN PESs. The PIP-NN approach deals with symmetry from the onset.

A disadvantage of NNs is the need for a non-linear fit. It makes the cost of fitting much higher than that of methods with only linear coefficients, such as PIP basis methods. The CPU and 
memory costs can be significant, e.g., for four-atom and larger molecules, NNs with hundreds of neurons and thousands of parameters are routinely used. Such fits may take hours, depending on hardware and software; however, the time required for the fit is small compared to the cost of computing the ab initio points. The cost issue is rapidly becoming less important as desktop computers with dozens of CPU cores and hundreds of GB of RAM become widespread. The number of parameters of a plain NN PES is actually small compared to that of some of the more complicated methods such as those using Coulomb matrix inspired inputs. ${ }^{50,61,130,135,136}$ Neural networks can accurately fit in internal coordinates of which the number is relatively small. This is not a trivial advantage; for example, using PIP based inputs leads to a very large number of variables; even when mitigated with the fundamental invariant approach, the number of inputs is quite large (dozens for a five-atom molecule). A very large number of inputs is also required by methods based on environment-dependent atomic contributions.

In terms of the accuracy of the potential, plain neural networks seem to be surpassed by methods like PIP basis, PIP-NN and PIP-IMLS. However, this underperformance has not been shown for the accuracy of observables computed on the PES. ${ }^{6}$ NNs have been shown to underperform only Gaussian process regression in terms of the error of the potential and of observables in controlled comparisons. ${ }^{215}$

Neural networks also have significant competitive advantages. The ease with which a sumof-products PES can be obtained with a neural network is unrivalled. As long as the data distribution is fairly uniform, accuracy can be improved (up to the limits imposed by the sampling density) simply by increasing the size a single hidden layer. NNs can easily work with large datasets as long as there is sufficient RAM; indeed, on modern desktop computers, we were able to easily fit millions of data within minutes. ${ }^{96}$ When the dataset is too large, data can be fitted in blocks, which can easily be done by fitting a subset of the data for a small number of cycles, which imposes only minor jaggedness of the convergence path in the parameter space. ${ }^{177}$ Alternatively, Kalman filter ${ }^{302,} 320$ like optimization methods can be used which, due to the sequential presentation of data, are not limited by data set size at all. This is not a trivial advantage; for example, the Gaussian process regression approach - to date the only PES fitting method that was shown in a controlled comparison to beat NNs in both potential and observables accuracy - scales badly with the sample size $N_{p t s}$, as one needs to compute the inverse of a $N_{p t s} \times N_{p t s}$ matrix which is used during fitting. Calling a GPR-fitted function is also expensive because a vector of size $N_{p t s}$ 
has to be used. Contrary to NNs, the GPR approach does not naturally allow splitting the data into blocks and becomes unstable when data points are too close, although approximations have been developed to allow using GPR to fit large data sets. ${ }^{322,323}$ Neural networks can work with large data sets of any distribution at all.

A significant, and perhaps the most important advantage of neural networks, which ensures them a prominent place in computational chemistry in the foreseeable future, is the fact that they can be integrated with other approaches, such as PIPs, HMDR, DTNN etc. In particular, approaches such as DTNN, PhysNet, SchNet which combine the advantages of Behler type NNs, of symmetry-adapted features as well as information contained in atomic numbers (nuclear charges) $Z$ have produced very powerful tools that can predict molecular properties beyond the PES and achieve record small errors for the PES. The idea of adding $Z$ to the list of inputs and fitting more than the PES (and maybe forces) is very powerful. Although costly, these approaches have already made it possible to build PESs with record-breaking accuracies and for relatively large molecules. They and other NN-based methods will surely continue to be useful for fitting PESs of small molecules and reactions.

As computing power and the availability of software have progressed, NN based PES fitting methods have become increasingly popular. This is true also for machine learning methods in computational chemistry and physics in general. They have made it possible to tackle some outstanding problems in computational chemistry, ${ }^{39,324,325}$ such as exchange-correlation ${ }^{326-328}$ and kinetic energy ${ }^{96,316,329,330}$ functional development, solution of the Schrödinger equation, ${ }^{331-333}$ and prediction of properties without solving the Schrödinger equation ${ }^{334-337}$ that require even more powerful hardware and software resources than PES fitting, and in addition, manpower with interdisciplinary knowledge. Due to the availability of these resources, these applications of ML in chemistry (some of which are reviewed in this issue) have recently been experiencing rapid growth. Using machine learning and specifically NN based methods to construct PESs led to conceptual and computational advances in the application of ML techniques to quantum chemistry data. In this sense, ML PES construction blazed the trail for the applications of NNs and ML in general in chemistry that we are now witnessing. 


\section{Acknowledgements}

T. C. was supported the Natural Sciences and Engineering Research Council of Canada.

\section{Abbreviations}

AIMD ab initio molecular dynamics

AM1 Austin Model 1

BO Born-Oppenheimer

(U)CCSD(T) (unrestricted) couple cluster with single and double substitutions and non-iterative triples

CPU central processing unit

DAF distributed approximating functional

DFT density functional theory

DFTB density functional tight binding

DOF degree of freedom

DTNN_deep tensor neural network

(M)EAM (modified) embedded atom model

EANN embedded atom neural network

ELM extreme learning machine

ERF Rydberg function approximation

FI fundamental invariant

GPR Gaussian process regression

HDMR high dimensional model representation

HDNNP high dimensional neural network potential

HIP-NN hierarchically interacting particle neural network

IMLS interpolating moving least squares

LM Levenberg-Marquardt

mae mean absolute error 


$\begin{array}{ll}\text { MCTDH } & \text { multiconfiguration time-dependent Hartree } \\ \text { MD } & \text { molecular dynamics } \\ \text { ME } & \text { moiety-energy } \\ \text { MEP } & \text { minimum energy path } \\ \text { MM } & \text { molecular mechanics } \\ \text { MP2 (MP4) } & 2^{\text {nd }}\left(4^{\text {th) }} \text { order Møller-Plesset perturbation theory }\right. \\ \text { MRCI } & \text { multireference configuration interaction } \\ \text { MSI } & \text { modified Sheppard interpolation } \\ \text { NN } & \text { neural network } \\ \text { NRPIP } & \text { non-redundant permutationally invariant polynomial } \\ \text { PCA } & \text { principal component analysis } \\ \text { PES } & \text { potential energy surface } \\ \text { PIP } & \text { permutationally invariant polynomial } \\ \text { PM6 } & \text { parameterization method 6 } \\ \text { RAM } & \text { random access memory } \\ \text { RBF } & \text { radial basis function } \\ \text { RKHS } & \text { reproducing kernel Hilbert space } \\ \text { rmse } & \text { root mean squared error } \\ \text { RS } & \text { random sampling } \\ \text { SDQ } & \text { single, double and quadruple } \\ \text { SMF } & \text { systematic molecular fragmentation } \\ \text { SOP } & \text { sum of products } \\ & \end{array}$

\section{References}

1. Werner, H.-J., Matrix - formulated direct multiconfiguration self - consistent field and multiconfiguration reference configuration - interaction methods. In Ab Initio Methods in Quantum Chemistry Part 2, Lawley, K. P., Ed. Wiley: 1987. 
2. Bartlett, R. J.; Musial, M., Coupled-cluster theory in quantum chemistry. Reviews of Modern Physics 2007, 79, 291.

3. $\quad$ Dawes, R.; Ndengue, S. A., Single- and multireference electronic structure calculations for constructing potential energy surfaces. International Reviews in Physical Chemistry 2016, 25, 441478.

4. Hase, W. L.; Song, K.; Gordon, M. S., Direct dynamics simulations. Computing in Science \& Engineering 2003, 5, 36-44.

5. Hohenberg, P.; Kohn, W., Inhomogeneous electron gas. Physical Review 1964, 136, B864-B871.

6. Kohn, W.; Sham, L. J., Self-consistent equations including exchange and correlation effects. Physical Review 1965, 140, A1133-A1138.

7. Kauppi, E., Ab initio - discrete variable representation calculation of vibrational energy levels. Journal of Chemical Physics 1996, 105, 7986.

8. Lasorne, B.; Worth, G. A.; Robb, M. A., Excited-state dynamics WIREs Computational Molecular Science 2011, 1, 460-475.

9. $\quad$ Lasorne, B.; Robb, M. A.; Worth, G. A., Direct quantum dynamics using variational multiconfiguration Gaussian wavepackets. Implementation details and test case. Physical Chemistry Chemical Physics 2007, 9, 3210-3227.

10. Murrell, J. N.; Carter, S.; Frantos, S.; Huxley, P.; Varandas, A. J. C., Molecular potential energy functions. Wiley: Toronto, 1984.

11. Truhlar, D. G.; Steckler, R.; Gordon, M. S., Potential energy surfaces for polyatomic reaction dynamics. Chemical Reviews 1987, 87, 217-236.

12. Kuhn, B.; Rizzo, T. R.; Luckhaus, D.; Quack, M.; Suhm, M., A new six-dimensional analytical potential up to chemically significant energies for the electronic ground state of hydrogen peroxide. Journal of Chemical Physics 1999, 111, 2565.

13. Marquardt, R.; Sagui, K.; Zheng, J.; Thiel, W.; Luckhaus, D.; Yurchenko, S.; Mariotti, F.; Quack, M., Global analytical potential energy surface for the electronic ground state of NH3 from high level ab initio calculations. Journal of Physical Chemistry A 2013, 117, 7502-7522.

14. LeRoy, R. J.; Henderson, R., A new potential function form incorporating extended longrange behaviour: application to ground-state $\mathrm{Ca}_{2}$ Molecular Physics 2007, 105, 663-677.

15. Mezey, P. G., Potential Energy Hypersurfaces. Elsevier: Amsterdam, 1987.

16. Collins, M. A., Molecular potential-energy surfaces for chemical reaction dynamics. Theoretical Chemistry Accounts 2002, 108, 313-324.

17. Ischtwan, J.; Collins, M. A., Molecular potential energy surfaces by interpolation. Journal of Chemical Physics 1994, 100, 8080.

18. Moyano, G. E.; Collins, M. A., Molecular potential energy surfaces by interpolation: Strategies for faster convergence. Journal of Chemical Physics 2004, 121, 9769.

19. Collins, M. A.; Zhang, D. H., Application of interpolated potential energy surfaces to quantum reactive scattering. Journal of Chemical Physics 111, 111, 9924.

20. Dawes, R.; Wagner, A. F.; Thompson, D. L., Ab initio wavenumber accurate spectroscopy: ${ }^{1} \mathrm{CH}_{2}$ and $\mathrm{HCN}$ vibrational levels on automatically generated IMLS potential energy surfaces. Journal of Physical Chemistry A 2009, 113, 4709-4721.

21. Guo, Y.; Thompson, D. L., Interpolating moving least-squares methods for fitting potential energy surfaces: An application to the H2CN unimolecular reaction. Journal of Chemical Physics 2007, 126, 104105. 
22. Guo, Y.; Kawano, A.; Thompson, D. L., Interpolating moving least-squares methods for fitting potential energy surfaces: Applications to classical dynamics calculations. Journal of Chemical Physics 2004, 121, 5091.

23. Braams, B. J.; Bowman, J. M., Permutationally invariant potential energy surfaces in high dimensionality. International Reviews in Physical Chemistry 2009, 28, 577-606.

24. Bowman, J. M.; Czako, G.; Fu, B., High-dimensional ab initio potential energy surfaces for reaction dynamics calculations. Physical Chemistry Chemical Physics 2011, 13, 8094-8111.

25. Gyori, T.; Czako, G., Automating the development of high-dimensional reactive potential energy surfaces with the ROBOSURFER program system. Journal of Chemical Theory and Computation 2020, 16, 51-66.

26. Majumder, M.; Ndengue, S.; Dawes, R., Automated construction of molecular potential energy surfaces. Molecular Physics 2016, 114, 1-18.

27. Qu, C.; Yu, Q.; Bowman, J. M., Permutationally invariant potential energy surfaces. Annual Review of Physical Chemistry 2018, 69, 151-175.

28. Hollebeek, T.; Ho, T.-S.; Rabitz, H., Constructing muldidimensional molecular potential energy suraces from ab initio data. Annual Review of Physical Chemistry 1999, 50, 537-570.

29. Ho, T.-S.; Rabitz, H., Reproducing kernel Hilbert space interpolation methods as a paradigm of high dimensional model representations: Application to multidimensional potential energy surface construction. Journal of Chemical Physics 2003, 119, 6433.

30. Szalay, V., Iterative and direct methods employing distributed approximating functionals for the reconstruction of a potential energy surface from its sampled values. Journal of Chemical Physics 1999, 111, 8804.

31. Hoffman, D. K.; Frishman, A.; Kouri, D. J., Distributed approximating functional approach to fitting multi-dimensional surfaces. Chemical Physics Letters 1996, 262, 393-399.

32. Hankins, D.; Moskowitz, J. W.; Stillinger, F. H., Water molecule interactions. Journal of Chemical Physics 1970, 53, 4544-4554.

33. Medders, G. R.; Babin, V.; Paesani, F., A critical assessment of two-body and three-body interactions in water. Journal of Chemical Theory and Computation 2013, 9, 1103-1114.

34. Carter, S.; Culik, S. J.; Bowman, J. M., Vibrational self-consistent field method for manymode systems: A new approach and application to the vibrations of $\mathrm{CO}$ adsorbed on $\mathrm{Cu}(100)$. Journal of Chemical Physics 1997, 107, 10458.

35. Carter, S.; Handy, N. C., On the representation of potential energy surfaces of polyatomic molecules in normal coordinates. Chemical Physics Letters 2002, 352, 1-7.

36. Rauhut, G., Efficient calculation of potential energy surfaces for the generation of vibrational wave functions. Journal of Chemical Physics 2004, 121, 9313.

37. Szalewicz, K., Symmetry-adapted perturbation theory of intermolecular forces. WIREs Computational Molecular Science 2012, 2, 254-272.

38. Babin, V.; Leforestier, C.; Paesani, F., Development of a "first principles” water potential with flexible monomers: Dimer potential energy surface, VRT spectrum, and second virial coefficient. Journal of Chemical Theory and Computation 2013, 9, 5395-5403.

39. Rupp, M., Machine learning for quantum mechanics in a nutshell. International Journal of Quantum Chemistry 2015, 115, 1058-1073.

40. Rasmussen, C. E.; Williams, C. K. I., Gaussian processes for machine learning. MIT Press: Cambridge MA, 2006.

41. Sacks, J.; Schiller, S. B.; J., W. W., Designs for computer experiments. Technometrics 1989, 31, 41-47. 
42. Cui, J.; Krems, R. V., Efficient non-parametric fitting of potential energy surfaces for polyatomic molecules with Gaussian processes. Journal of Physics B: Atomic, Molecular and Optical Physics 2016, 49, 224001.

43. Beck, M. H.; Jaeckle, A.; Worth, G. A.; Meyer, H.-D., The multiconfiguration timedependent Hartree (MCTDH) method: a highly efficient algorithm for propagating wavepackets. Physics Reports 2000, 324, 1-105.

44. Bowman, J. M.; Carrington, T.; Meyer, H.-D., Variational quantum approaches for computing vibrational energies of polyatomic molecules. Molecular Physics 2008, 106, 21452182.

45. Carrington, T., Computing (ro-)vibrational spectra of molecules with more than four atoms. Journal of Chemical Physics 2017, 146, 120902.

46. Krasnoshchekov, S. V.; Vogt, N.; Stepanov, N. F., Ab initio anharmonic analysis of vibrational spectra of uracil using the numerical-analytic implementation of operator Van Vleck perturbation theory. Journal of Physical Chemistry A 2015, 119, 6723-6737.

47. Thomas, P. S.; Carrington, T.; Agarwal, J.; Schaefer, H. F. I., Using an iterative eigensolver and intertwined rank reduction to compute vibrational spectra of molecules with more than a dozen atoms: Uracil and naphthalene. Journal of Chemical Physics 2018, 149, 064108.

48. McCoy, A. B.; Huang, X.; Carter, S.; Landeweer, M. Y.; Bowman, J. M., Fulldimensional vibrational calculations for $\mathrm{H}_{5} \mathrm{O}_{2}{ }^{+}$using an ab initio potential energy surface. Journal of Chemical Physics 2005, 122, 061101.

49. Pandey, A.; Poirier, B., An algorithm to find (and plug) "holes" in multi-dimensional surfaces. Journal of Chemical Physics 2020, 152, 214102.

50. Behler, J.; Parinello, M., Generalized neural-network representation of high-dimensional potential-energy surfaces. Physical Revoew Letters 2007, 98, 146401.

51. Bholoa, A.; Kenny, S. D.; Smith, R., A new approach to potential fitting using neural networks. Nuclear Instruments and Methods in Physics Research B 2007, 255, 1-7.

52. Behler, J.; Martonak, R.; Donadio, D.; Parinello, M., Pressure-induced phase transitions in silicon studied by neural network-based metadynamics simulations. Physica Status Solidi $B$ 2008, 245, 2618-2629.

53. Behler, J., Atom-centered symmetry functions for constructing high-dimensional neural network potentials. Journal of Chemical Physics 2011, 134, 074106.

54. Zhang, Y.; Hu, C.; Jiang, B., Embedded atom neural network potentials: Efficient and accurate machine learning with a physically inspired representation. Journal of Physical Chemistry Letters 2019, 10, 4962-4967.

55. Jovan Jose, K. V.; Artrith, N.; Behler, J., Construction of high-dimensional neural network potentials using environment-dependent atom pairs. Journal of Chemical Physics 2012, 136, 194111.

56. Artrith, N.; Hiller, B.; Behler, J., Neural network potentials for metals and oxides - First applications to copper clusters at zinc oxide. Physica Status Solidi B 2012, 250, 1191-1203.

57. Behler, J., Neural network potential-energy surfaces in chemistry: a tool for large-scale simulations. Physical Chemistry Chemical Physics 2011, 13, 17930-17955.

58. Behler, J.; Martonak, R.; Donadio, D.; Parinello, M., Metadynamics simulations of the high-pressure phases of silicon employing a high-dimensional neural network potential. Physical Review Letters 2008, 100, 185501.

59. Huang, Y.; Kang, J.; Goddard, W. A., III ; Wang, L.-W., Density functional theory based neural network force fields from energy decompositions. Physical Review B 2019, 99, 064103. 
60. Hu, Q.; Weng, M.; Chen, X.; Li, S.; Pan, F.; Wang, L.-W., Neural network force fields for metal growth based on energy decompositions. Journal of Physical Chemistry Letters 2020, 11, 1364-1369.

61. Schutt, K. T.; Arbabzadah, F.; Chmiela, S.; Muller, K. S.; Tkatchenko, A., Quantumchemical insights from deep tensor neural networks. Nature Communications 2017, 8, 13890.

62. Smith, J. S.; Isayev, O.; Roitberg, A. E., ANI-1: an extensible neural network potential with DFT accuracy at force field computational cost. Physical Chemistry Chemical Physics 2017, 8, 3192-3203S.

63. Min, K.; Cho, E., Neural network interatomic potential for predicting the formation of planar defect in nanocrystal. Journal of Physical Chemistry C 2020, 124, 9424-9433.

64. Morawietz, T.; Sharma, V.; Behler, J., A neural network potential-energy surface for the water dimer based on environment-dependent atomic energies and charges. Journal of Chemical Physics 2012, 136, 064103.

65. Natarajan, S. K.; Morawietz, T.; Behler, J., Representing the potential-energy surface of protonated water clusters by high-dimensional neural network potentials. Physical Chemistry Chemical Physics 2015, 17, 8356-8371.

66. Nguyen, T. T.; Szekely, E.; Imbalzano, G.; Behler, J.; Csanyi, G.; Ceriotti, M.; Gotz, A. W.; Paesani, F., Comparison of permutationally invariant polynomials, neural networks, and Gaussian approximation potentials in representing water interactions through many-body expansions. Journal of Chemical Physics 2018, 148, 241725.

67. Donoho, D., High-dimensional data analysis: The curses and blessings of dimensionality. In AMS Conference on Math Challenges of the 21st Century, AMS: 2000; pp 1-32.

68. Majumber, M.; Hegger, S. E.; Dawes, R.; Manzhos, S.; Wang, X.-G.; Carrington, T.; Li, J.; Guo, H., Explicitly-correlated MRCI-F12 potential energy surfaces for methane fit with several permutation invariant schemes and full-dimensional vibrational calculations. Molecular Physics 2015, 113, 1823-1833.

69. Manzhos, S.; Carrington, T.; Laverdure, L.; Mosey, N., Computing the anharmonic vibrational spectrum of $\mathrm{UF}_{6}$ in 15 dimensions with an optimized basis set and rectangular collocation Journal of Physical Chemistry A 2015, 119, 9557-9567.

70. Widrow, B.; Lehr, M. A., 30 years of adaptive neural networks: perceptron, madaline, and backpropagation. Proceedings of the IEEE 1990, 78, 1415-1442.

71. Zupan, J.; Gasteiger, J., Neural networks: A new method for solving chemical problems or just a passing phase? Analytica Chimica Acta 1991, 248.

72. $\quad$ Neural Networks: Tricks of the Trade. Springer: Berlin, 1998.

73. Rocha, M.; Cortez, P.; Neves, J., Evolution of neural networks for classification and regression. Neurocomputing 2007, 70, 2809-2816.

74. Conical intersections: Theory, computation, and experiment. World Scientific: Singapore, 2011; p 768.

75. Kolmogorov, A. N., On the representations of continuous functions of many variables by superpositions of continuous functions of one variable and addition. Doklady Akademii Nauk USSR 1957, 114, 953-956.

76. Hornik, K., Approximation capabilities of muitilayer feedforward networks. Neural Networks 1991, 4, 251-257.

77. Scarselli, F.; Tsoi, A. C., Universal Approximation Using Feedforward Neural Networks: A Survey of Some Existing Methods, and Some New Results. Neural Networks 1998, 11, 15-37. 
78. Kurkova, V., Kolmogorov's theorem and multilayer neural networks. Neural Networks 1992, 5, 501-506.

79. Cybenko, G., Approximation by superpositions of a single function Mathematics of Control, Signals and Systems 1989, 2, 303-314.

80. Funahashi, K., On the approximate realization of continuous mappings by neural networks. Neural Networks 1989, 2, 183-192.

81. Sprecher, D. A., On the structure of continuous functions of several variables. Transactions of the American Mathematical Society 1965, 115, 340-355.

82. Nees, M., Approximative versions of Kolmogorov's superposition theorem, proved constructively. Journal of Computational and Applied Mathematics 1994, 54.

83. Hornik, K.; Stinchcombe, M.; White, H., Universal Approximation of an Unknown Mapping and Its Derivatives Using Multilayer Feedforward Networks. Neural Networks 1990, 3, 551-560.

84. Katsuura, H.; Sprecher, D. A., Computational aspects of Kolmogorov's superposition theorem. Neural Networks 1994, 7, 455-461.

85. Sprecher, D. A., A numerical implementation of Kolmogorov's superpositions. Neural Networks 1996, 9, 765-772.

86. Sprecher, D. A., A numetical implementation of Kolmogorov's superpositions II. Neural Networks 1997, 10, 447-457.

87. Sprecher, D. A., An improvement in the superposition theorem of Kolmogorov. Journal of Mathematical Analysis and Applications 1972, 38, 208-213.

88. Sprecher, D. A., A Universal Mapping for Kolmogorov's Superposition Theorem. Neural Networks 1993, 6, 1089-1094.

89. Sprecher, D. A.; Draghici, S., Space-filling curves and Kolmogorov superposition-based neural network. Neural Networks 2002, 15, 57-67.

90. Pinkus, A.; Maiorov, V., Lower bounds for approximation by MLP neural networks. Neurocomputing 1999, 25, 81-91.

91. Gorban, A. N., Approximation of continuous functions of several variables by an arbitrary nonlinear continuous function of one variable, linear functions, and their superpositions. Applied Mathematics Letters 1998, 11, 45-49.

92. Barron, A. R., Universal approximation bounds for superpositions of a sigmoidal function. IEEE Transactions on Information Theory 1993, 39, 930-945.

93. Liao, Y.; Fang, S.-C.; Nuttle, H. L. W., Relaxed conditions for radial-basis function networks to be universal approximators. Neural Networks 2003, 16, 1019-1028.

94. Wu, W.; Nan, D.; Long, J.-l.; Ma, Y.-m., A comment on “Relaxed conditions for radialbasis function networks to be universal approximators". Neural Networks 2008, 21, 1464-1465.

95. Mulero-Martinez, J. I., Functions bandlimited in frequency are free of the curse of dimensionality. Neurocomputing 2007, 70, 1439-1452.

96. Golub, P.; Manzhos, S., Kinetic energy densities based on the fourth order gradient expansion: performance in different classes of materials and improvement via machine learning. Physical Chemistry Chemical Physics 2019, 21, 378-395.

97. Zhou, Z.-H.; Wu, J.; Tang, W., Ensembling neural networks: Many could be better than all. Artificial Intelligence 2002, 137, 239-263.

98. Manzhos, S.; Dawes, R.; Carrington, T., Neural network based approaches for building high dimensional and quantum dynamics-friendly potential energy surfaces. International Journal of Quantum Chemistry 2015, 115, 1012-1020. 
99. Handley, C. M.; Popelier, P. L. A., Potential energy surfaces fitted by artificial neural networks. Journal of Physical Chemistry A 2010, 114, 3371-3383.

100. Manzhos, S.; Carrington, T., Using neural networks to represent potential surfaces as sums of products. Journal of Chemical Physics 2006, 125, 194105.

101. Koch, W.; Zhang, D. H., Separable potential energy surfaces from multiplicative artificial neural networks. Journal of Chemical Physics 2014, 141, 021101.

102. Schmitt, M., On the complexity of computing and learning with multiplicative neural networks. Neural Computation 2001, 14, 241-301.

103. Hervas-Martinez, C.; Martinez-Estudillo, F. J.; Carbonero-Ruz, M., Multilogistic regression by means of evolutionary product-unit neural networks. Neural Networks 2008, 21, 951-961.

104. Pinkus, A., Approximation theory of the MLP model in neural networks. Acta Numerica 1999, 8, 143-195.

105. Leshno, M.; Lin, V. Y.; Pinkus, A.; Schocken, S., Multilayer feedforward networks with a nonpolynomial activation function can approximate any function Neural Networks 1993, 6, 861867.

106. Hinton, G. E.; Salakhutdinov, R. R., Reducing the dimensionality of data with neural networks. Science 2006, 313, 504-507.

107. Kiarashinejad, Y.; Abdollahramezani, S.; Adibi, A., Deep learning approach based on dimensionality reduction for designing electromagnetic nanostructures. npg Computational Materials 2020, 6, 12.

108. Carter, S.; Bowman, J. M., Variational Calculations of Rotational-Vibrational Energies of CH4 and Isotopomers Using an Adjusted ab Initio Potential. Journal of Physical Chemistry A 2000, 104, 2355-2361.

109. Carter, S.; Bowman, J. M.; Harding, L. B., Ab initio calculations of force fields for $\mathrm{H}_{2} \mathrm{CN}$ and $\mathrm{ClHCN}$ and vibrational energies of $\mathrm{H}_{2} \mathrm{CN}$. Spectrochimica Acta A 1997, 53, 1179-1188.

110. Li, G.; Rosenthal, C.; Rabitz, H., High Dimensional Model Representations. Journal of Physical Chemistry A 2001, 105, 7765-7777.

111. Rabitz, H.; Alis, O. F., General foundations of high - dimensional model representations. Journal of Mathematical Chemistry 1999, 25, 197-233.

112. Wang, S.-W.; Georgopoulos, P. G.; Li, G.; Rabitz, H., Random Sampling-High Dimensional Model Representation (RS-HDMR) with nonuniformly distributed variables: Application to an integrated multimedia/multipathway exposure and dose model for trichloroethylene. Journal of Physical Chemistry A 2003, 107, 4707-4716.

113. Li, G.; Wang, S.-W.; Rabitz, H., Practical approaches to construct RS-HDMR component functions. Journal of Physical Chemistry A 2002, 106, 8721-8733.

114. Manzhos, S.; Carrington, T., A Random-Sampling High Dimensional Model Representation neural network for building potential energy surfaces. Journal of Chemical Physics 2006, 125, 084109.

115. Manzhos, S.; Carrington, T., Using redundant coordinates to represent potential energy surfaces with lower-dimensional functions. Journal of Chemical Physics 2007, 127, 014103.

116. Manzhos, S.; Yamashita, K.; Carrington, T., Fitting sparse multidimensional data with low-dimensional terms Computer Physics Communications 2009, 180, 2002-2012.

117. Manzhos, S.; Yamashita, K.; Carrington, T., Extracting functional dependence from sparse data using dimensionality reduction: Application to potential energy surface construction. In 
Coping with Complexity: Model Reduction and Data Analysis, Gorban, A. N.; Roose, D., Eds. Spribger Verlag: Berlin, 2011; pp 133-149.

118. Behler, J., Constructing high - dimensional neural network potentials: A tutorial review. International Journal of Quantum Chemistry 2015, 115, 1032-1050.

119. Behler, J., Representing potential energy surfaces by high-dimensional neural network potentials. Journal of Physics: Condensed Matter 2014, 26, 183001.

120. Behler, J.; Lorenz, S.; Reuter, K., Representing molecule-surface interactions with symmetry-adapted neural networks. Journal of Chemical Physics 2007, 127, 014705.

121. Zhang, Y.; Zhou, X.; Jiang, B., Bridging the gap between direct dynamics and globally accurate reactive potential energy surfaces using neural networks. Journal of Physical Chemistry Letters 2019, 10, 1185-1191.

122. Kolb, B.; Luo, X.; Zhou, X.; Jiang, B.; Guo, H., High-dimensional atomistic neural network potentials for molecule-surface interactions: $\mathrm{HCl}$ scattering from $\mathrm{Au}(111)$. Journal of Physical Chemistry Letters 2017, 8, 666-672.

123. Gerrits, N.; Shakouri, K.; Behler, J.; Kroes, G.-J., Accurate probabilities for highly activated reaction of polyatomic molecules on surfaces using a high-dimensional neural network potential: $\mathrm{CHD}_{3}+\mathrm{Cu}(111)$. Journal of Physical Chemistry Letters 2019, 10, 1763-1768.

124. Liu, Q.; Zhou, X.; Zhou, L.; Zhang, Y.; Luo, X.; Guo, H., Constructing high-dimensional neural network potential energy surfaces for gas-surface scattering and reactions. Journal of Physical Chemistry C 2018, 122, 1761-1769.

125. del Cueto, M.; Zhou, X.; Zhou, L.; Zhang, Y.; Jiang, B.; Guo, H., New perspectives on $\mathrm{CO} 2-\mathrm{Pt}(111)$ interaction with a high-dimensional neural network potential energy surface. Journal of Physical Chemistry C 2020, 124, 5174-5181.

126. Daw, M. S.; Baskes, M., Embedded-atom method: Derivation and application to impurities, surfaces and other defects in metals. Physical Review Letters 1984, 29, 6443.

127. Baskes, M., Application of the embedded-atom method to covalent materials: A semiempirical potential for silicon. Physical Review Letters 1987, 59, 2666.

128. Ghasemi, S. A.; Hofstetter, A.; Saha, S.; Goedecker, S., Interatomic potentials for ionic systems with density functional accuracy based on charge densities obtained by a neural network. Physical Review B 2015, 92, 045131.

129. Hafizi, R.; Ghasemi, S. A.; Hashemifar, S. J.; Akbarzadeh, H., A neural-network potential through charge equilibration for WS2: From clusters to sheets. Journal of Chemical Physics 2017, 147, 234306.

130. Schutt, K. T.; Sauceda, H. E.; Kindermans, P.-J.; Tkatchenko, A.; Muller, K. S., SchNet - A deep learning architecture for molecules and materials. Journal of Chemical Physics 2018, 148, 241722.

131. Rupp, M.; Tkatchenko, A.; Muller, K. R.; von Lilienfeld, O. A., Fast and accurate modeling of molecular atomization energies with machine learning. Physical Review Letters 2012, 108, 058301

132. Lu, Q.; Liu, Q.; Sun, Q.; Hsieh, C.-Y.; Zhang, S.; Shi, L.; Lee, C.-K., Deep learning for optoelectronic properties of organic semiconductors. Journal of Physical Chemistry C 2020, 124, 7048-7060.

133. Ramakrishnan, R.; Dral, P. O.; Rupp, M.; Von Lilienfeld, O. A., Quantum chemistry structures and properties of 134 kilo molecules. Scientific Data 2014, 1, 140022. 
134. Lu, J.; Wang, C.; Zhang, Y., Predicting molecular energy using force-field optimized geometries and atomic vector representations learned from an improved deep tensor neural network. Journal of Chemical Theory and Computation 2019, 15, 4113-4121.

135. Unke, O. T.; Meuwly, M., PhysNet: A neural network for predicting energies, forces, dipole moments, and partial charges. Journal of Chemical Theory and Computation 2019, 15, 3678-3693.

136. Lubbers, N.; Smith, J. S.; Barros, K., Hierarchical modeling of molecular energies using a deep neural network. Journal of Chemical Physics 2018, 148, 241715.

137. Muller, T.; Hernandez, A.; Wang, C., Machine learning for interatomic potential models. Journal of Chemical Physics 2020, 152, 050902.

138. Le, H. M.; Raff, L. M., Cis $\rightarrow$ trans, trans $\rightarrow$ cis isomerizations and $\mathrm{N}-\mathrm{O}$ bond dissociation of nitrous acid (HONO) on an ab initio potential surface obtained by novelty sampling and feedforward neural network fitting. Journal of Chemical Physics 2008, 128, 194310.

139. Castro, E.; Avila, G.; Manzhos, S.; Agarwal, J.; Schaefer, H. F.; Carrington, T., Applying a Smolyak collocation method to $\mathrm{Cl}_{2} \mathrm{CO}$. Molecular Physics 2017, 115, 1775-1785.

140. Le, H. M.; Huynh, S.; Raff, L. M., Molecular dissociation of hydrogen peroxide (HOOH) on a neural network ab initio potential surface with a new configuration sampling method involving gradient fitting. Journal of Chemical Physics 2009, 131, 014107.

141. Le, H. M.; Raff, L. M., Molecular dynamics investigation of the bimolecular reaction BeH $+\mathrm{H}_{2}->\mathrm{BeH}_{2}+\mathrm{H}$ on an ab Initio potential-energy surface obtained using neural network methods with both potential and gradient accuracy determination. Journal of Physical Chemistry A 2010, $114,45-53$.

142. Li, J.; Jiang, B.; Guo, H., Permutation invariant polynomial neural network approach to fitting potential energy surfaces. II. Four-atom systems. Journal of Chemical Physics 2013, 139, 204103.

143. Li, A.; Guo, H., A full-dimensional global potential energy surface of $\mathrm{H}_{3} \mathrm{O}+\left(\tilde{a}^{3} \mathrm{~A}\right)$ for the $\mathrm{OH}+\left(\tilde{\mathrm{X}}^{3} \Sigma^{-}\right)+\mathrm{H}_{2}\left(\tilde{\mathrm{X}}^{1} \Sigma_{\mathrm{g}}{ }^{+}\right) \rightarrow \mathrm{H}\left({ }^{2} \mathrm{~S}\right)+\mathrm{H}_{2} \mathrm{O}+\left(\tilde{\mathrm{X}}^{2} \mathrm{~B}_{1}\right)$ reaction. Journal of Physical Chemistry A 2014, $118,11168-11176$.

144. Chen, J.; Xu, X.; Xu, X.; Zhang, D. H., An accurate global potential energy surface for the $\mathrm{OH}+\mathrm{CO} \rightarrow \mathrm{H}+\mathrm{CO}_{2}$ reaction using neural networks. Journal of Chemical Physics 2013, 138 , 221104.

145. Xu, X.; Chen, J.; Liu, S.; Zhang, D. H., An ab initio-based global potential energy surface for the $\mathrm{SH}_{3}$ system and full-dimensional state-to-state quantum dynamics study for the $\mathrm{H}_{2}+\mathrm{HS}$ $>\mathrm{H}_{2} \mathrm{~S}+\mathrm{H}$ reaction. Journal of Computational Chemistry 2019, 40, 1151-1160.

146. Latino, D. A. R. S.; Freitas, F. F. M.; Aires-de-Sousa, J.; Fernandes, F. M. S. S., Neural networks to approach potential energy surfaces: Application to a molecular dynamics simulation. International Journal of Quantum Chemistry 2007, 107, 2120-2132.

147. Cooper, A. M.; Hallmen, P. P.; Kastner, J., Potential energy surface interpolation with neural networks for instanton rate calculations. Journal of Chemical Physics 2018, 148, 094106.

148. Hobday, S.; Smith, R.; Belbruno, J., Applications of neural networks to fitting interatomic potential functions. Modelling and Simulation in Materials Science and Engineering 1999, 7, 397412.

149. Tersoff, J., New empirical model for the structural properties of silicon. Physical Review Letters 1986, 56, 632. 
150. Tersoff, J., Empirical interatomic potential for silicon with improved elastic properties. Physical Review B 1988, 38, 9902.

151. Malshe, M.; Narulkar, R.; Raff, L. M.; Hagan, M.; Bukkapatnum, S.; Komanduri, R., Parametrization of analytic interatomic potential functions using neural networks. Journal of Chemical Physics 2008, 129, 044111.

152. Raff, L. M.; Malshe, M.; Hagan, M.; Doughan, D. I.; Rockley, M. G.; Komanduri, R., $\mathrm{Ab}$ initio potential-energy surfaces for complex, multichannel systems using modified novelty sampling and feedforward neural networks. Journal of Chemical Physics 2005, 122, 084104.

153. Purja Pun, G. P.; Batra, R.; Ramprasad, R.; Mishin, Y., Physically informed artificial neural networks for atomistic modeling of materials. Nature Communications 2019, 10, 2339.

154. Gassner, H.; Probst, M.; Lauenstein, A.; Hermansson, K., Representation of Intermolecular Potential Functions by Neural Networks. Journal of Physical Chemistry A 1998, 102, 4596-4605.

155. Lorenz, S.; Scheffler, M.; Gross, A., Descriptions of surface chemical reactions using a neural network representation of the potential-energy surface. Physical Review B 2006, 73.

156. Ludwig, J.; Vlachos, D. G., Ab initio molecular dynamics of hydrogen dissociation on metal surfaces using neural networks and novelty sampling. Journal of Chemical Physics 2007, $127,154716$.

157. Law, M. M.; Fraser-Smith, J.; Perotto, C. U., The potential energy surface of isomerising disilynew. Physical Chemistry Chemical Physics 2012, 14, 6922-6936.

158. Jiang, B.; Guo, H., Permutation invariant polynomial neural network approach to fitting potential energy surfaces. Journal of Chemical Physics 2013, 139, 054112.

159. Shao, K.; Chen, J.; Zhao, Z.; Zhang, D. H., Fitting potential energy surfaces with fundamental invariant neural network. Journal of Chemical Physics 2016, 145, 071101.

160. Prudente, F. V.; Acioli, P. H.; Soares Neto, J. J., The fitting of potential energy surfaces using neural networks: Application to the study of vibrational levels of $\mathrm{H}_{3}{ }^{+}$. Journal of Chemical Physics 1998, 109.

161. Nguyen, H. T. T.; Le, H. M., Modified feed-forward neural network structures and combined-function-derivative approximations incorporating exchange symmetry for potential energy surface fitting. Journal of Physical Chemistry A 2012, 116, 4629-4638.

162. Manzhos, S.; Wang, X.-G.; Dawes, R.; Carrington, T., A nested molecule-independent neural network approach for high-quality potential fits. Journal of Physical Chemistry A 2006, 110, 5295 - 5304 .

163. Manzhos, S.; Yamashita, K., A model for the dissociative adsorption of $\mathrm{N}_{2} \mathrm{O}$ on $\mathrm{Cu}(100)$ using a continuous potential energy surface. Surface Science 2010, 604, 555-561.

164. Xu, X.; Chen, J.; Zhang, D. H., Global potential energy surface for the $\mathrm{H}+\mathrm{CH}_{4}>\mathrm{H}_{2}+\mathrm{CH}_{3}$ reaction using neural networks. Chinese Journal of Chemical Physics 2014, 27, 373-379.

165. Manzhos, S.; K., N.; Yamashita, K., Three-body interactions in clusters CO-(pH2)n. Chemical Physics Letters 2010, 493, 229-233.

166. Yao, K.; Herr, J. E.; Parkhill, J., The many-body expansion combined with neural networks. Journal of Chemical Physics 2017, 146, 014106.

167. Le, A. T. H.; Vu, N. H.; Dinh, T. S.; Cao, T. M.; Le, H. M., Molecular dynamics investigations of chlorine peroxide dissociation on a neural network ab initio potential energy surface. Theoretical Chemistry Accounts 2012, 131, 1158.

168. Chen, J.; Xu, X.; Xu, X.; Zhang, D. H., A global potential energy surface for the $\mathrm{H}_{2}+\mathrm{OH}$ $\leftrightarrow \mathrm{H}_{2} \mathrm{O}+\mathrm{H}$ reaction using neural networks. Journal of Chemical Physics 2013, 138, 154301. 
169. Lorenz, S.; Gross, A.; Scheffler, M., Representing high-dimensional potential-energy surfaces for reactions at surfaces by neural networks. Chemical Physics Letters 2004, 395, 210215.

170. Jiang, B.; Guo, H., Six-dimensional quantum dynamics for dissociative chemisorption of $\mathrm{H}_{2}$ and $\mathrm{D}_{2}$ on $\mathrm{Ag}(111)$ on a permutation invariant potential energy surface. Physical Chemistry Chemical Physics 2014, 16, 24704-24715.

171. Jiang, B.; Guo, H., Permutation invariant polynomial neural network approach to fitting potential energy surfaces. III. Molecule-surface interactions. Journal of Chemical Physics 2014, $141,034109$.

172. Huang, X.; Braams, B. J.; Bowman, J. M.; Kelly, R. E. A.; Tennyson, J.; Groenenboom, G. C.; van der Avoird, A., New ab initio potential energy surface and the vibration-rotationtunneling levels of $\left(\mathrm{H}_{2} \mathrm{O}\right)_{2}$ and $\left(\mathrm{D}_{2} \mathrm{O}\right)_{2}$. Journal of Chemical Physics 2008, 128, 034312.

173. Czako, G.; Shepler, B. C.; Braams, B. J.; Bowman, J. M., Accurate ab initio potential energy surface, dynamics, and thermochemistry of the $\mathrm{F}+\mathrm{CH}_{4} \rightarrow \mathrm{HF}+\mathrm{CH}_{3}$ reaction. Journal of Chemical Physics 2009, 130, 084301.

174. Huang, X.; Braams, B. J.; Bowman, J. M., Ab initio potential energy and dipole moment surfaces for $\mathrm{H}_{5} \mathrm{O}_{2}+$. Journal of Chemical Physics 2005, 122, 044308.

175. Malshe, M.; Raff, L. M.; Hagan, M.; Bukkapatnum, S.; Komanduri, R., Input vector optimization of feed-forward neural networks for fitting ab initio potential-energy databases. Journal of Chemical Physics 2010, 132, 204103.

176. Foresman, J. B.; Frisch, A., Exploring Chemistry with Electronic Structure Methods. Gaussian, Inc.: Pittsburgh, PA, 1996.

177. Manzhos, S.; Carrington, T., Using neural networks, optimized coordinates and high dimensional model representations to obtain a vinyl bromide potential surface. Journal of Chemical Physics 2008, 129, 224104.

178. Prudente, F. V.; Soares Neto, J. J., The fitting of potential energy surfaces using neural networks. Application to the study of the photodissociation processes. Chemical Physics Letters 1998, 287, 585-589.

179. Blank, T. B.; Brown, S. D.; Calhoun, A. W.; Doren, D. J., Neural network models of potential energy surfaces. Journal of Chemical Physics 1995, 103, 4129-4137.

180. Brown, D. F. R.; Gibbs, M. N.; Clary, D. C., Combining ab initio computations, neural networks, and diffusion Monte Carlo: An efficient method to treat weakly bound molecules. Journnal of Chemical Physics 1996, 105, 7597-7604.

181. Duch, W.; Diercksen, G. H. F., Neural networks as tools to solve problems in physics and chemistry. Computer Physics Communications 1994, 82, 91-103.

182. Tafeit, E.; Estelberger, W.; Horejsi, R.; Moeller, R.; Oettl, K.; Vrecko, K.; Reibnegger, G., Neural networks as a tool for compact representation of ab initio molecular potential energy surfaces. Journal of Molecular Graphics 1996, 14, 12-18.

183. No, K. T.; Chang, B. H.; Kim, S. Y.; Jhon, M. S.; Scherega, H. A., Description of the potential energy surface of the water dimer with an artificial neural network. Chemical Physics Letters 1997, 271, 152-156.

184. Rocha Filho, T. M.; Oliveira, Z. T.; Malbouisson, L. A. C.; Gargano, R.; Soares Neto, J. J., The use of neural networks for fitting potential energy surfaces: A comparative case study for the $\mathrm{H}_{3}{ }^{+}$molecule. International Journal of Quantum Chemistry 2003, 95, 281-288. 
185. Raff, L. M.; Malshe, M.; Hagan, M.; Doughan, D. I.; Rockley, M. G.; Komanduri, R., Ab initio potential-energy surfaces for complex, multichannel systems using modified novelty sampling and feedforward neural networks. Journal of Chemical Physics 2005, 122, 084194.

186. Agrawal, P. M.; Raff, L. M.; Hagan, M.; Komanduri, R., Molecular dynamics investigations of the dissociation of $\mathrm{SiO}_{2}$ on an ab initio potential energy surface obtained using neural network methods. Journal of Chemical Physics 2006, 124, 134306.

187. Malshe, M.; Raff, L. M.; Rockley, M. G.; Hagan, M.; Agrawal, P. M.; Komanduri, R., Theoretical investigation of the dissociation dynamics of vibrationally excited vinyl bromide on an ab initio potential-energy surface obtained using modified novelty sampling and feedforward neural networks. II. Numerical application of the method. Journal of Chemical Physics 2007, 127, 134105.

188. Pukrittayakamee, A.; Malshe, M.; Hagan, M.; Raff, L. M.; Narulkar, R.; Bukkapatnum, S.; Komanduri, R., Simultaneous fitting of a potential-energy surface and its corresponding force fields using feedforward neural networks. Journal of Chemical Physics 2009, 130, 134101.

189. Malshe, M.; Narulkar, R.; Raff, L. M.; Hagan, M.; Bukkapatnum, S.; Agrawal, P. M.; Komanduri, R., Development of generalized potential-energy surfaces using many-body expansions, neural networks, and moiety energy approximations. Journal of Chemical Physics 2009, 130, 184102.

190. Latino, D. A. R. S.; Fartaria, R. P. S.; Freitas, F. F. M.; Aires-de-Sousa, J.; Silva Fernandes, F. M. S., Mapping potential energy surfaces by neural networks: The ethanol/Au(1 1 1) interface. Journal of Electroanalytical Chemistry 2008, 624, 109-120.

191. No, K. T.; Chang, B. H.; Kim, S. Y.; Jhon, M. S.; Scheraga, H. A., Description of the potential energy surface of the water dimer with an artificial neural network. Chemical Physics Letters 1997, 271, 152-156.

192. Møller, C.; Plesset, M. S., Note on an approximation treatment for many-electron systems. Physical Review 1934, 46, 0618-0622.

193. Press, W. H.; Teukolsky, S. A.; Vetterling, W. T.; Flannery, B. P., Numerical Recipes: The Art of Scientific Computing. Cambridge University Press: Hong Kong, 2007.

194. Nguyen, H. T. T.; Le, H. M., An implementation of the Levenberg-Marquardt algorithm forsimultaneous-energy-gradient fitting using two-layer feed-forwardneural networks. Chemical Physics Letters 2015, 629, 40-45.

195. Nguyen, H. T. T.; Thi, C. M.; Le, H. M., Theoretical investigations of BBS (singlet)?BSB (triplet) transformation on a potential energy surface obtained from neural network fitting. Chemical Physics 2013, 426, 31-37.

196. Raghavachari, K.; Pople, J. A., Approximate 4th-order perturbation-theory of electron correlation energy. International Journal of Quantum Chemistry 1978, 14, 91-100.

197. Doughan, D. I.; Raff, L. M.; Rockley, M. G.; Hagan, M.; Agrawal, P. M.; Komanduri, R., Theoretical investigation of the dissociation dynamics of vibrationally excited vinyl bromide on an ab initio potential-energy surface obtained using modified novelty sampling and feedforward neural networks. Journal of Chemical Physics 2006, 124, 054321.

198. Jiang, B.; Li, J.; Guo, H., Potential energy surfaces from high fidelity fitting of ab initio points: the permutation invariant polynomial - neural network approach. International Reviews in Physical Chemistry 2016, 35, 479-506.

199. Szalay, P. G.; Muller, T.; Gidofalvi, G.; Lischka, H.; Shepard, R., Multiconfiguration Self-Consistent Field and Multireference Configuration Interaction methods and applications. Chemical Reviews 2012, 112, 108-181. 
200. Li, A.; Guo, H., A nine-dimensional ab initio global potential energy surface for the $\mathrm{H}_{2} \mathrm{O}^{+}$ $+\mathrm{H}_{2} \rightarrow \mathrm{H}_{3} \mathrm{O}^{+}+\mathrm{H}$ reaction. Journal of Chemical Physics 2014, 140, 224313.

201. Liu, Y.; Bai, M.; Song, H.; Xie, D.; Li, J., Anomalous kinetics of the reaction between $\mathrm{OH}$ and $\mathrm{HO} 2$ on an accurate triplet state potential energy surface. Physical Chemistry Chemical Physics 2019, 21, 12667-12675.

202. Lu, X.; Shao, K.; Fu, B.; Wang, X.; Zhang, D. H., An accurate full-dimensional potential energy surface and quasiclassical trajectory dynamics of the $\mathrm{H}+\mathrm{H}_{2} \mathrm{O}_{2}$ two-channel reaction. Physical Chemistry Chemical Physics 2018, 20, 23095.

203. Lu, X.; Meng, Q.; Wang, X.; Fu, B.; Zhang, D. H., Rate coefficients of the $\mathrm{H}^{+} \mathrm{H}_{2} \mathrm{O}_{2}->$ $\mathrm{H}_{2}+\mathrm{HO}_{2}$ reaction on an accurate fundamental invariant-neural network potential energy surface. Journal of Chemical Physics 2018, 149, 174303.

204. Lu, X.; Wang, X.; Fu, B.; Zhang, D. H., Theoretical investigations of rate coefficients of $\mathrm{H}+\mathrm{H}_{2} \mathrm{O}_{2} \rightarrow \mathrm{OH}+\mathrm{H}_{2} \mathrm{O}$ on a full-dimensional potential energy surface. Journal of Physical Chemistry A 2019, 123, 3969-3976.

205. Schran, C.; Behler, J.; Marx, D., Automated fitting of neural network potentials at coupled cluster accuracy: Protonated water clusters as testing ground. Journal of Chemical Theory and Computation 2020, 16, 88-92.

206. Allinger, N. L.; Yuh, Y. H.; Lii, J. H., Molecular mechanics. The MM3 force field for hydrocarbons. Journal of The American Chemical Society 1989, 111, 8551-8566.

207. Dewar, M. J. S.; Zoebisch, E. G.; Healy, E. F., AM1: A new general purpose quantum mechanical molecular model. Journal of The American Chemical Society 1985, 107, 3902-3909.

208. Stewart, J. J. P., Optimization of parameters for semiempirical methods. V. Modification of NDDO approximations and application to 70 elements. Journal of Molecular Modeling 2007, 13, 1173-1213.

209. Gastegger, M.; Marquetand, P., High-dimensional neural network potentials for organic reactions and an improved training algorithm. Journal of Chemical Theory and Computation 2015, 11, 2187-2198.

210. Sanville, E.; Bholoa, A.; Smith, R.; Kenny, S. D., Silicon potentials investigated using density functional theory fitted neural networks. Journal of Physics: Condensed Matter 2008, 20 , 285219.

211. Chen, X.; Goldsmith, C. F., Accelerating variational transition state theory via artificial neural networks. Journal of Physical Chemistry A 2020, 124, 1038-1046.

212. Rivero, U.; Unke, O. T.; Meuwly, M.; Willitsch, S., Reactive atomistic simulations of Diels-Alder reactions: The importance of molecular rotations. Journal of Chemical Physics 2019, 151, 104301.

213. Boes, J. R.; Kitchin, J. R., Neural network predictions of oxygen interactions on a dynamic Pd surface. Molecular Simulation 2017, 43, 346-354.

214. Bittencourt, A. C. P.; Prudente, F. V.; Vianna, J. D. M., The fitting of potential energy and transition moment functions using neural networks: transition probabilities in $\mathrm{OH}\left(\mathrm{A}^{2} \Sigma^{+} \rightarrow \mathrm{X}^{2} \Pi\right)$. Chemical Physics 2004, 297, 153-161.

215. Kamath, A.; Vargas-Hernández, R. A.; Krems, R. V.; Carrington, T.; Manzhos, S., Neural Networks vs Gaussian Process regression for representing potential energy surfaces: a comparative study of fit quality and vibrational spectrum accuracy. Journal of Chemical Physics 2018, 148, 241702. 
216. Avila, G.; Carrington, T., A multi-dimensional Smolyak collocation method in curvilinear coordinates for computing vibrational spectra. Journal of Chemical Physics 2015, 143, 214108

217. Li, J.; Chen, J.; Zhang, D. H.; Guo, H., Quantum and quasi-classical dynamics of the $\mathrm{OH}$ $+\mathrm{CO} \rightarrow \mathrm{H}+\mathrm{CO}_{2}$ reaction on a new permutationally invariant neural network potential energy surface. Journal of Chemical Physics 2014, 140, 044327.

218. Zhang, Z.; Chen, J.; Yang, M.; Zhang, D. H., Time-dependent wave packet study of the $\mathrm{H}_{2}+\mathrm{CH}_{3} \rightarrow \mathrm{H}+\mathrm{CH}_{4}$ reaction. Journal of Physical Chemistry A 2015, 119, 12480-12484.

219. Lu, D.; Li, J., Full-dimensional global potential energy surfaces describing abstraction and exchange for the $\mathrm{H}+\mathrm{H}_{2} \mathrm{~S}$ reaction. Journal of Chemical Physics 2016, 145, 014303.

220. Song, Q.; Zhang, Q.; Meng, Q., Neural-network potential energy surface with small database and high precision: A benchmark of the $\mathrm{H}+\mathrm{H}_{2}$ system. Journal of Chemical Physics 2019, 151, 114302.

221. Shen, X.; Zhang, Z.; Zhang, D. H., CH4 dissociation on Ni(111): a quantum dynamics study of lattice thermal motion†. Physical Chemistry Chemical Physics 2015, 17, 25499-25504.

222. Shen, X.; Chen, J.; Zhang, Z.; Shao, K.; Zhang, D. H., Methane dissociation on Ni(111): A fifteen-dimensional potential energy surface using neural network method. Journal of Chemical Physics 2015, 143, 144701.

223. Liu, T.; Fu, B.; Zhang, D. H., HCl dissociating on a rigid Au(111) surface: A sixdimensional quantum mechanical study on a new potential energy surface based on the RPBE functional. Journal of Chemical Physics 2017, 146, 164706.

224. Liu, T.; Fu, B.; Zhang, D. H., Six-dimensional potential energy surfaces of the dissociative chemisorption of $\mathrm{HCl}$ on $\mathrm{Ag}(111)$ with three density functionals. Journal of Chemical Physics 2018, 149, 054702.

225. Liu, T.; Fu, B.; Zhang, D. H., Six-dimensional potential energy surfaces for the dissociative chemisorption of $\mathrm{HCl}$ on rigid $\mathrm{Ag}(100)$ and $\mathrm{Ag}(110)$ surfaces. Journal of Chemical Physics 2019, 151, 144707.

226. Manthe, U.; Meyer, H.-D.; Cederbaum, L. S., Wave - packet dynamics within the multiconfiguration Hartree framework: General aspects and application to NOCl. Journal of Chemical Physics 1992, 87, 3199.

227. Meyer, H.-D.; Manthe, U.; Cederbaum, L. S., The multi-configurational time-dependent Hartree approach. Chemical Physics Letters 1990, 167, 73-78.

228. Lecrerc, A.; Carrington, T., Calculating vibrational spectra with sum of product basis functions without storing full-dimensional vectors or matrices. Journal of Chemical Physics 2014, $140,174111$.

229. Thomas, P. S.; Carrington, T., An intertwined method for making low-rank, sum-ofproduct basis functions that makes it possible to compute vibrational spectra of molecules with more than 10 atoms. Journal of Chemical Physics 2017, 146, 204110.

230. Thomas, P. S.; Carrington, T., Using nested contractions and a hierarchical tensor format to compute vibrational spectra of molecules with seven atoms. Journal of Physical Chemistry A 2015, 119, 13074-13091.

231. Larsson, H. R., Computing vibrational eigenstates with tree tensor network states (TTNS). Journal of Chemical Physics 2019, 151, 204102.

232. Rakhuba, M.; Oseledets, I., Calculating vibrational spectra of molecules using tensor train decomposition. Journal of Chemical Physics 2016, 145, 124101 
233. Ziegler, B.; Rauhut, G., Efficient generation of sum-of-products representations of highdimensional potential energy surfaces based on multimode expansions. Journal of Chemical Physics 2016, 144, 114114.

234. Avila, G.; Carrington, T., Using multi-dimensional Smolyak interpolation to make a sumof-products potential. Journal of Chemical Physics 2015, 143, 044106.

235. Jaeckle, A.; Meyer, H.-D., Product representation of potential energy surfaces. Journal of Chemical Physics 1996, 104, 7974.

236. De Lathauwer, L.; De Moor, B.; Vandewalle, J., A multilinear singular value decomposition SIAM Journal on Matrix Analysis and Applications 2000, 21, 1253-1278.

237. Kolda, T. G.; Bader, B. W., Tensor decompositions and applications SIAM Review 2009, 51, 455-500.

238. Schroeder, M., Transforming high-dimensional potential energy surfaces into a canonical polyadic decomposition using Monte Carlo methods. Journal of Chemical Physics 2020, 152, 024108.

239. Peláez, D.; Meyer, H.-D., The multigrid POTFIT (MGPF) method: Grid representations of potentials for quantum dynamics of large systems. Journal of Chemical Physics 2013, 138, 014108 240. Schröder, M.; Meyer, H.-D., Transforming high-dimensional potential energy surfaces into sum-of-products form using Monte Carlo methods. Journal of Chemical Physics 2017, 147, 064105

241. de Silva, V.; Lim, L.-H., Tensor rank and the ill-posedness of the best low-rank approximation problem. SIAM Journal of Matrix Analysis and Applications 2008, 30, 1084-1127. 242. Pradhan, E.; Cerreon-Macedo, J.-L.; Cuervo, J. E.; Schroeder, M.; Brown, A., Ab initio potential energy and dipole moment surfaces for CS2: Determination of molecular vibrational energies. Journal of Physical Chemistry A 2013, 117, 6925-6931.

243. Pradhan, E.; Brown, A., Neural network exponential fitting of a potential energy surface with multiple minima: Application to HFCO. Journal of Molecular Spectroscopy 2016, 330, 158164.

244. Pradhan, E.; Brown, A., Vibrational energies for HFCO using a neural network sum of exponentials potential energy surface. Journal of Chemical Physics 2016, 144, 174305.

245. Pradhan, E.; Brown, A., A ground state potential energy surface for HONO based on a neural network with exponential fitting functions. Physical Chemistry Chemical Physics 2017, 19, 22272-22281.

246. Brown, A.; Pradhan, E., Fitting potential energy surfaces to sum-of-products form with neural networks using exponential neurons. Journal of Theoretical and Computational Chemistry 2017, 16, 1730001.

247. Andersson, K.; Malmqvist, P.-Å.; Roos, B. O.; Sadlej, A. J.; Wolinski, K., Second-order perturbation theory with a CASSCF reference function. Journal of Physical Chemistry 1990, 94, 5483.

248. Domcke, W.; Yarkony, D. R., Role of conical intersections in molecular spectroscopy and photoinduced chemical dynamics. Annual Review of Physical Chemistry 2012, 63, 325-352.

249. Zhu, X.; Yarkony, D. R., Non-adiabaticity: the importance of conical intersections Molecular Physics 2016, 114, 1983-2013.

250. Koeppel, H.; Domcke, W.; Cederbaum, L. S., Multimode molecular dynamics beyond the Born - Oppenheimer approximation. Advances in Chemical Physics 1984, 57 (LVII), 59. 
251. Lenzen, T.; Eisfeld, W.; Manthe, U., Vibronically and spin-orbit coupled diabatic potentials for $\mathrm{X}\left({ }^{2} \mathrm{P}\right)+\mathrm{CH}_{4} \rightarrow \mathrm{HX}+\mathrm{CH}_{3}$ reactions: Neural network potentials for $\mathrm{X}=\mathrm{Cl}$. Journal of Chemical Physics 2019, 150, 244115.

252. Lenzen, T.; Manthe, U., Neural network based coupled diabatic potential energy surfaces for reactive scattering. Journal of Chemical Physics 2017, 147, 084105.

253. Williams, D. M. G.; Eisfeld, W., Neural network diabatization: A new ansatz for accurate high-dimensional coupled potential energy surfaces. Journal of Chemical Physics 2018, 149, 204106.

254. Williams, D. M. G.; Viel, A.; Eisfeld, W., Diabatic neural network potentials for accurate vibronic quantum dynamics - The test case of planar $\mathrm{NO}_{3}$. Journal of Chemical Physics 2019, 151, 164118.

255. Guan, Y.; Zhang, D. H.; Guo, H.; Yarkony, D. R., Representation of coupled adiabatic potential energy surfaces using neural network based quasi-diabatic Hamiltonians: $1,2^{2} \mathrm{~A}$ ' states of LiFH. Physical Chemistry Chemical Physics 2019, 21, 14205-14213.

256. Guan, Y.; Guo, H.; Yarkony, D. R., Neural network based quasidiabatic Hamiltonians with symmetry adaptation and a correct description of conical intersections. Journal of Chemical Physics 2019, 150, 214101.

257. Guan, Y.; Fu, B.; Zhang, D. H., Construction of diabatic energy surfaces for LiFH with artificial neural networks. Journal of Chemical Physics 2017, 147, 224307.

258. Yin, Z.; Guan, Y.; Fu, B.; Zhang, D. H., Two-state diabatic potential energy surfaces of ClH2 based on nonadiabatic couplings with neural networks. Physical Chemistry Chemical Physics 2019, 21, 20372-20383.

259. Jasper, A. W.; Hack, M. D.; Truhlar, D. G., Coupled quasidiabatic potential energy surfaces for LiFH. Journal of Chemical Physics 2002, 116, 8353.

260. Xie, C.; Zhu, X.; Yarkony, D. R.; Guo, H., Permutation invariant polynomial neural network approach to fitting potential energy surfaces. IV. Coupled diabatic potential energy matrices. Journal of Chemical Physics 2018, 149, 144107.

261. Guan, Y.; Guo, H.; Yarkony, D. R., Extending the representation of multistate coupled potential energy surfaces to include properties operators using neural networks: Application to the $1,2^{1} \mathrm{~A}$ states of ammonia. Journal of Chemical Theory and Computation 2020, 16, 302-313.

262. Guan, Y.; Yarkony, D. R., Accurate neural network representation of the ab initio determined spin-orbit interaction in the diabatic representation including the effects of conical intersections. Journal of Physical Chemistry Letters 2020, 11, 1848-1858.

263. Westermayr, J.; Gastegger, M.; Marquetand, P., Combining SchNet and SHARC: The SchNarc machine learning approach for excited-state dynamics. Journal of Physical Chemistry Letters 2020, 11, 3828-3834.

264. Xu, W.; Liu, X.; Luan, S.; Zhang, Q.; Zhang, P., An ab initio potential energy surface of the $\mathrm{He}+\mathrm{H}_{2} \rightarrow \mathrm{HeH}+\mathrm{H}$ reaction. Chemical Physics Letters 2008, 464, 92-96.

265. Yang, Z.; Wang, S.; Yuan, J.; Chen, M., Neural network potential energy surface and dynamical isotope effects for the $\mathrm{N}^{+}\left({ }^{3} \mathrm{P}\right)+\mathrm{H}_{2} \rightarrow \mathrm{NH}^{+}+\mathrm{H}$ reaction. Physical Chemistry Chemical Physics 2019, 21, 22203-22214.

266. Cho, K.-H.; No, K. T.; Scheraga, H. A., A polarizable force field for water using an artificial neural network. Journal of Molecular Structure 2002, 641, 77-91.

267. Busnengo, H. F.; Salin, A.; Dong, W., Representation of the 6D potential energy surface for a diatomic molecule near a solid surface. Journal of Chemical Physics 2000, 112. 
268. Brown, W. M.; Martin, S.; Pollock, S. N.; Coutsias, E. A.; Watson, J.-P., Algorithmic dimensionality reduction for molecular structure analysis. Journal of Chemical Physics 2008, 129, 064118.

269. Fukumizu, K.; Bach, F. R.; Jordan, M. I., Dimensionality reduction for supervised learning with Reproducing Kernel Hilbert Spaces. Journal of Machine Learning Research 2004, 5, 73-99.

270. Michielssens, S.; van Erp, T. S.; Kutzner, C.; Ceulemans, A.; de Groot, B. L., Molecular dynamics in principal component space. Journal of Physical Chemistry B 2012, 8350-8354.

271. Roweis, S. T.; Saul, L. K., Nonlinear dimensionality reduction by locally linear embedding. Science 2000, 290.

272. Cox, T.; Cox, M., Multidimensional scaling. Chapman \& Hall: London, 1994.

273. Herr, J. E.; Koh, K.; Yao, K.; Parkhill, J., Compressing physics with an autoencoder: Creating an atomic species representation to improve machine learning models in the chemical sciences. Journal of Chemical Physics 2019, 151, 084103.

274. Cubuk, E. D.; Malone, B. D.; Onat, B.; Waterland, A.; Kaxiras, E., Representations in neural network based empirical potentials. Journal of Chemical Physics 2017, 147, 024104.

275. Garashchuk, S.; Light, J. C., Quasirandom distributed Gaussian bases for bound problems. Journal of Chemical Physics 2001, 114, 3929.

276. Ku, J.; Kamath, A.; Carrington, T.; Manzhos, S., Machine learning optimization of the collocation point set for solving the Kohn-Sham eaquation. Journal of Physical Chemistry A 2019, 123, 10631-10642.

277. Manzhos, S.; Carrington, T.; Yamashita, K., Calculating anharmonic vibrational frequencies of molecules adsorbed on surfaces directly from ab initio energies with a moleculeindependent method: H2O on Pt(111). Surface Science 2011, 605, 616-622.

278. Chan, M.; Manzhos, S.; Carrington, T.; Yamashita, K., Parameterized bases for calculating vibrational spectra directly from ab initio data using rectangular collocation. Journal of Chemical Theory and Computation 2012, 8, 2053-2061.

279. Chan, M.; Carrington, T.; Manzhos, S., Anharmonic vibrations of the carboxyl group in acetic acid on TiO2: implications for adsorption mode assignment in dye-sensitized solar cells. Physical Chemistry Chemical Physics 2013, 15, 10028-10034.

280. Manzhos, S.; Chan, M.; Carrington, T., Favorable dimensionality scaling of rectangular collocation with adaptable basis functions up to 7 dimensions Journal of Chemical Physics 2013, 139, 051101.

281. Manzhos, S.; Carrington, T., Using an internal coordinate basis and a space-fixed Cartesian coordinate kinetic energy operator to compute a vibrational spectrum with Gaussian basis functions and rectangular collocation. Journal of Chemical Physics 2016, 145, 224110.

282. Manzhos, S.; Wang, X.-G.; Carrington, T., A multimode-like scheme for selecting the centers of Gaussian basis functions when computing vibrational spectra. Chemical Physics 2018, 509, 139-144.

283. Sobol, I. M., On the distribution of points on a cube and the approximate evaluation of integrals. USSR Computational Mathematics and Mathematical Physics 1967, 7, 86-112.

284. Elstner, M.; Porezag, D.; Jungnickel, G.; Elsner, J.; Haugk, M.; Frauenheim, T.; al., e., Self-consistent-charge density-functional tight-binding method for simulations of complex materials properties. Physical Review B 1998, 58, 7260.

285. M., G.; Cui, Q.; Elstner, M., DFTB3: Extension of the self-consistent-charge DensityFunctional Tight-Binding method (SCC-DFTB) Journal of Chemical Theory and Computation 2011, 7, 931-948. 
286. Lin, Q.; Zhang, Y.; Zhao, B.; Jiang, B., Automatically growing global reactive neural network potential energy surfaces: A trajectory-free active learning strategy. Journal of Chemical Physics 2020, 152, 154104.

287. Gastegger, M.; Schwiedrzik, L.; Bittermann, M.; Berzsenyi, F.; Marquetand, P., wACSF-Weighted atom-centered symmetry functions as descriptors in machine learning potentials. Journal of Chemical Physics 2018, 148, 241709.

288. Tokmanov, I. V.; Wagner, A. F.; Minkoff, M.; Thompson, D. L., Gradient incorporation in one-dimensional applications of interpolating moving least-squares methods for fitting potential energy surfaces. Theoretical Chemistry Accounts 2007, 118, 755-767.

289. Maisuradze, G. G.; Thompson, D. L., Interpolating moving least-squares methods for fitting potential energy surfaces: Illustrative approaches and applications. Journal of Physical Chemistry A 2003, 107, 7118-7124.

290. Maisuradze, G. G.; Thompson, D. L., Interpolating moving least-squares methods for fitting potential energy surfaces: Detailed analysis of one-dimensional applications. Journal of Chemical Physics 2003, 119, 10002.

291. Guo, Y.; Tokmanov, I. V.; Thompson, D. L.; Wagner, A. F.; Minkoff, M., Interpolating moving least-squares methods for fitting potential energy surfaces: Improving efficiency via local approximants. Journal of Chemical Physics 2007, 127, 214106.

292. Kawano, A.; Tokmanov, I. V.; Thompson, D. L., Interpolating moving least-squares methods for fitting potential-energy surfaces: Further improvement of efficiency via cutoff strategies. Journal of Chemical Physics 124, 124, 054105.

293. Wang, X.-G.; Carrington, T., New ideas for using contracted basis functions with a Lanczos eigensolver for computing vibrational spectra of molecules with four or more atoms. Journal of Chemical Physics 2002, 117, 6923.

294. Tremblay, J. C.; Carrington, T., Calculating vibrational energies and wave functions of vinylidene using a contracted basis with a locally reorthogonalized coupled two-term Lanczos eigensolver. Journal of Chemical Physics 2006, 125, 094311.

295. Light, J. C.; Carrington, T., Discrete - variable representations and their utilization. Prigogine, I.; Rice, S. A., Eds. Wiley: 2000; p 263.

296. Wang, X.-G.; Carrington, T., A finite basis representation Lanczos calculation of the bend energy levels of methane. Journal of Chemical Physics 2003, 118, 6946.

297. Manzhos, S.; Yamashita, K.; Carrington, T., On the advantages of a rectangular matrix collocation equation for computing vibrational spectra from small basis sets. Chemical Physics Letters 2011, 511, 434-439.

298. Manzhos, S.; Carrington, T.; Yamashita, K., Non-spectral methods for solving the Schroedinger equation for electronic and vibrational problems. Journal of Physical Chemistry Letters 2011, 2, 2193-2199.

299. Manzhos, S.; Carrington, T., Using rectangular collocation with finite difference derivatives to solve electronic Schrödinger equation. Journal of Chemical Physics 2018, 149, 204105.

300. Carter, S.; Handy, N. C.; Demaison, J., The rotational levels of the ground vibrational state of formaldehyde. Molecular Physics 1997, 90, 729-738.

301. Bartok, A. P.; Payne, M. C.; Kondor, R.; Csanyi, G., Gaussian approximation potentials: The accuracy of quantum mechanics, without the electrons. Physical Review Letters 2010, 104, 136403. 
302. Gastegger, M.; Kauffmann, C.; Behler, J.; Marquetand, P., Comparing the accuracy of highdimensional neural network potentials and the systematic molecular fragmentation method: A benchmark study for all-trans alkanes. Journal of Chemical Physics 2016, 144, 194110.

303. Collins, M. A.; Deev, V. A., Accuracy and efficiency of electronic energies from systematic molecular fragmentation

(2006). Journal of Chemical Physics 2006, 125, 104104.

304. Collins, M. A., Systematic fragmentation of large molecules by annihilation Physical Chemistry Chemical Physics 2012, 14, 7744-7751.

305. Li, J.; Song, K.; Behler, J., A critical comparison of neural network potentials for molecular reaction dynamics with exact permutation symmetry. Physical Chemistry Chemical Physics 2019, 21, 9672-9682.

306. MATLAB 2019a, The MathWorks, Inc.: Natick, Massachusetts, United States, 2019.

307. Eaton, J. W.; Bateman, D.; Hauberg, S.; Wehbring, R. GNU Octave version 5.1.0 manual: a high-level interactive language for numerical computations, 2019.

308. R: A Language and Environment for Statistical Computing, R Foundation for Statistical Computing: Vienna, Austria, 2017.

309. Python Language Reference, version 2.7., Python Software Foundation.

310. Pedregosa, F.; Varoquaux, G.; Gramfort, A.; Michel, V.; Thirion, B.; Grisel, O.; Blondel, M.; Prettenhofer, P.; Weiss, R.; Dubourg, V.; Vanderplas, J.; Passos, A.; Cournapeau, D.; Brucher, M.; Perrot, M.; Duchesnay, E., Scikit-learn: Machine Learning in Python. Journal of Machine Learning Research 2011, 12, 2825-2830.

311. Behler, J. RuNNer - a neural network code for high-dimensional potential-energy surfaces Lehrstuhl für Theoretische Chemie, Ruhr-Universität Bochum: Bochum.

312. Lee, K.; Yoo, D.; Jeong, W.; Han, S., SIMPLE-NN: An efficient package for training and executing neural-network interatomic potentials. Computer Physics Communications 2019, 242, 95-103.

313. Singraber, A.; Behler, J.; Dellago, C., Library-based LAMMPS implementation of highdimensional neural network potentials. Journal of Chemical Theory and Computation 2019, 15, 1827-1840.

314. Rumelhart, D. E.; Hinton, G. E.; Williams, R. J., Learning representations by backpropagating errors. Nature 1986, 323, 533-536.

315. Kingma, D. P.; Ba, J., Adam: A method for stochastic optimization. In arXiv:1412.6980 2014.

316. Fujinami, M.; Kageyama, R.; Seino, J.; Ikabata, Y.; Nakai, H., Orbital-free density functional theory calculation applying semi-local machine-learned kinetic energy density functional and kinetic potential. Chemical Physics Letters 2020, 748, 137358.

317. Misawa, M.; Fukushima, S.; Koura, A.; Shimamura, K.; Shimojo, F.; Tiwari, S.; Nomura, K.-I.; Kalia, R. K.; Nakano, A.; Vashishta, P., Application of first-principles-based artificial neural network potentials to multiscale-shock dynamics simulations on solid materials. Journal of Physical Chemistry Letters 2020, 11, 4536-4541.

318. Huang, G.-B.; Zhu, Q.-Y.; Siew, C.-K., Extreme learning machine: Theory and applications. Neurocomputing 2006, 70, 489-501.

319. Zhang, Y.; Zhou, X.; Jiang, B., Accelerating the construction of neural network potential energy surfaces: A fast hybrid training algorithm. Chinese Journal of Chemical Physics 2017, 30, 727-734. 
320. Kalman, R. E., A new approach to linear filtering and prediction problems ASME Journal of Basic Engineering 1960, 82, 34-45.

321. Legrain, F.; Manzhos, S., Highly accurate local pseudopotentials of $\mathrm{Li}, \mathrm{Na}$, and $\mathrm{Mg}$ for Orbital Free Density Functional Theory. Chemical Physics Letters 2015, 622, 99-103.

322. Csató, L.; Opper, M., Sparse on-line Gaussian processes. Neural Computation 2002, 14 , 641-668.

323. Quiñonero Candela, J.; Rasmussen, C. E. A., Unifying view of sparse approximate Gaussian process regression. Journal of Machine Learning Research 2005, 6, 1939-1959.

324. Dral, P. O., Quantum chemistry in the age of machine learning. Journal of Physical Chemistry Letters 2020, 11, 2336-2347.

325. Schleder, G. R.; Padilha, A. C. M.; Acosta, C. M.; Costa, M.; Fazzio, A., From DFT to machine learning: Recent approaches to materials science - a review. Journal of Physics: Materials 2019, 2, 032001.

326. Nagai, R.; Akashi, R.; Sugino, O., Completing density functional theory by machine learning hidden messages from molecules. npg Computational Materials 2020, 6, 43.

327. Kolb, B.; Lentz, L. C.; Kolpak, A. M., Discovering charge density functionals and structure-property relationships with PROPhet: A general framework for coupling machine learning and first principles methods. Scientific Reports 2017, 7, 1192.

328. Schmidt, J.; Benavides-Riveros, C. L.; Marques, M. A. L., Machine Learning the Physical Nonlocal Exchange-Correlation Functional of Density-Functional Theory. Journal of Physical Chemistry Letters 2019, 10, 6425-6431.

329. Seino, J.; Kageyama, R.; Fujinami, M.; Ikabata, Y.; Nakai, H., Semi-local machinelearned kinetic energy density functional demonstrating smooth potential energy curves. Chemical Physics Letters 2019, 734, 136732.

330. Li, L.; Snyder, J. C.; Pelaschier, I. M.; Huang, J.; Niranjan, U. N.; Duncan, P.; Rupp, M.; Müller, K. R.; Burke, K., Understanding machine-learned density functionals. International Journal of Quantum Chemistry 2016, 116, 819-833.

331. Manzhos, S., Machine learning for the solution of the Schrödinger equation. Machine Learning Science and Technology 2020, 1, 013002.

332. Dral, P. O.; von Lilienfeld, O. A.; Thiel, W., Machine learning of parameters for accurate semiempirical quantum chemical calculations. Journal of Chemical Theory and Computation 2015, $11,2120-2125$.

333. Schütt, K. T.; Gastegger, M.; Tkatchenko, A.; Muller, K. R.; Maurer, R. J., Unifying machine learning and quantum chemistry with a deep neural network for molecular wavefunctions. Nature Communications 2019, 10, 5024.

334. Ramprasad, R.; Batra, R.; Pilania, G.; Mannodi-Kanakkithodi, A.; Kim, C., Machine learning in materials informatics: Recent applications and prospects. npg Computational Materials 2017, 3, 54.

335. Butler, K. T.; Davies, D. W.; Cartwright, H.; Isayev, O.; Walsh, A., Machine learning for molecular and materials science. Nature 2018, 559, 547-555.

336. Chandrasekaran, A.; Kamal, D.; Batra, R.; Kim, C.; Chen, L.; Ramprasad, R., Solving the electronic structure problem with machine learning. npg Computational Materials 2019, 5, 22. 337. Hase, F.; Kreisbeck, C.; Aspuru-Guzik, A., Machine learning for quantum dynamics: deep learning of excitation energy transfer properties. Chemical Science 2017, 8, 8419-8426. 